Vierter Beratungsgegenstand:

\title{
Die Entwicklung eines Internationalen Verwaltungsrechts als Aufgabe der Rechtswissenschaft
}

1. Bericht von Professor Dr. Claus Dieter Classen, Greifswald*

Inhalt

I. Einführung ..................... 367

II. „Vertikale“ Internationalisierung der Verwaltung . . . . . . 369

1. Empirische Ebene ... . . . . . . . . . . . . . . . . 369

2. Die Stellung der Staaten gegenüber internationaler Verwaltung ................. 373

3. Internationale Verwaltung und nationales Recht ... 375

a) Organisationsrecht .............. 375

b) Innerstaatliche Bedeutung internationaler Standards . 376

aa) Legitimationsbezogene Fragen . . . . . . . . 378

bb) Rechtsstaatliche Fragen . . . . . . . . . . . . 384

4. Individualbezogene Maßnahmen internationaler Verwaltung .................. 385

a) Rechtsstaatliche Standards auf internationaler Ebene ................... . 385

b) Rückgriff auf nationale Strukturen . . . . . . . . . 390

c) Insbesondere: der Austausch personenbezogener Daten ................... 391

III. Horizontale Internationalisierung der Verwaltung . . . . . 392

1. Anknüpfungen und Verweisungen ......... 392

a) Anknüpfung an ausländische Sachverhalte . . . . . 392

b) Anknüpfung an und Verweisung auf ausländische Rechtslagen . . . . . . . . . . . . . . . . 394

\footnotetext{
* Für kritische Durchsicht früherer Fassungen und hilfreiche Hinweise danke ich Georg Nolte, Armin Hatje, Uwe Kischeh Michael Rodi und meiner Frau.
} 
2. Behördenkooperation . . . . . . . . . . . 398

a) Abstimmung zwischen Behörden verschiedener Staaten . . . . . . . . . . . . . . . . 398

b) Amts- und Vollstreckungshilfe . . . . . . . . 400

3. Grenzüberschreitende Aktivitäten . . . . . . . . . 401

a) Tätigkeit ausländischer Behörden im Inland . . . . . 401

b) Tätigkeit inländischer Behörden im Ausland . . . . . 405

IV. Die Aufgabe der Rechtswissenschaft . . . . . . . . 405 


\section{Einfuihrung}

Die vielfach diskutierte Globalisierung macht auch vor der Verwaltung nicht halt. Über das listing-Verfahren des UN-Sicherheitsrates etwa wurde gestern gesprochen. ${ }^{1}$ Nun ist für das deutsche Verfassungsrecht die von Klaus Vogel im Jahre 1964 propagierte „offene Staatlichkeit“2 weitgehend Allgemeingut geworden. Deren Konsequenzen für die Verwaltung wurden dagegen - abgesehen von der hier ausgeklammerten ${ }^{3}$ Europäisierung ${ }^{4}$ - von der deutschen Staatsrechtslehre bislang nur wenig erörtert. ${ }^{5}$ Dies ist bedauerlich, denn Ansätze zu einer Verwaltung auf internationaler Ebene gibt es seit langem. 1865 wurde die Internationale Telegraphen-Union ${ }^{6}$ gegründet, neun Jahre später der Weltpostverein ${ }^{7}$. In Folge des Wandels des Völkerrechts von einem Regelset zur Bewältigung zwischenstaatlicher Konflikte hin zu einer kooperativen Gestal-

1 Dazu G. Nolte in diesem Band, II.1.

2 Die Verfassungsentscheidung des Grundgesetzes für eine internationale Zusammenarbeit, 1964, passim.

${ }^{3}$ Das Recht von EG und EU nimmt wegen des Vorrangs von Gemeinschaftsrecht gegenüber dem nationalen Recht sowie verfassungsrechtlich mit Blick auf Art. 23 GG insgesamt eine Sonderstellung ein. Hinzu kommen ein die Staaten übergreifendes Rechtssystem (zur Bindung der Mitgliedstaaten an die allgemeinen Rechtsgrundsätze auch bei Durchführung des Unionsrechts EuGH, Rs C-354/04 P, EuGRZ 2007, 448 Gestorias pro amnistía u.a., Rn. 51, sowie Rs C-303/05, EuGRZ 2007, 273 - Advocaten voor de Wereld, Rn. 45) und gemeinsame institutionelle Strukturen. Anders der Ansatz im Begleitaufsatz von C. Ohler DVB1. 2007, 1083.

${ }^{4}$ Dazu insbesondere T. v. Danwitz Verwaltungsrechtliches System und Europäische Integration, 1996; S. Kadelbach Allgemeines Verwaltungsrecht unter europäischem Einfluss, 1999; A. Hatje Die gemeinschaftsrechtliche Steuerung der Wirtschaftsverwaltung, 1998; M. Zuleeg/H.-W. Rengeling Deutsches und europäisches Verwaltungsrecht, VVDStRL 53 (1994), 154ff. bzw. $202 \mathrm{ff}$; E. Pache/T. Groß Verantwortung und Effizienz in der Mehrebenenverwaltung, VVDStRL 66 (2007), $106 \mathrm{ff}$. bzw. $152 \mathrm{ff}$.

5 Umfassend aber vor allem C. Tietje Internationalisiertes Verwaltungshandeln, 2001; ferner etwa K. König VA 92 (2001), $475 \mathrm{ff}$.; $R$. Wahl in: ders., Verfassungsstaat, Europäisierung, Internationalisierung, 2003, $17 \mathrm{ff}$., $53 \mathrm{ff}$.; $E$. Schmidt-Aßmann FS R. Schmidt, 2006, 149 ff.; ders. Der Staat 45 (2006) $315 \mathrm{ff.;}$ C. Möllers/A. Voßkuhle/C. Walter (Hrsg.) Internationales Verwaltungsrecht, 2007; aus internationaler Sicht B. Kingsbury/N. Krisch' R. B. Stewart Law and Contemporary Problems 68 (2005), $15 \mathrm{ff}$.

6 Siehe heute BGB1. 1996 II 1308; dazu A. Tegge Internationale TelekommunikationsUnion, 1994.

7 Gegründet als „General Postal Union“ erhielt er vier Jahre später den heute noch gültigen Namen „Universal Postal Union“. Heutige Vertragsfassung: BGBl 1998 II 2085. Dazu Tietje (Fn. 5) $449 \mathrm{ff}$; J.D. Aston Sekundärgesetzgebung internationaler Organisationen zwischen mitgliedstaatlicher Souveränität und Gemeinschaftsdisziplin, $2005,126 \mathrm{ff}$. 
tungsordnung 8 übernehmen heute zahlreiche internationale Organisationen Verwaltungsaufgaben, beeinflussen aber vor allem die Arbeit der nationalen Verwaltungen. Dieses Phänomen wird nachfolgend als ,vertikale“ Internationalisierung bezeichnet. Der Begriff wird allerdings nur mit Anführungszeichen verwendet, da die Staaten kollektiv „Herren“ der jeweiligen Verträge sind und individuell „Herren“ der Entscheidung über die Mitgliedschaft in einer Organisation.9 Daneben steht die horizontale Internationalisierung der Verwaltung - die Öffnung einer staatlichen Verwaltung gegenüber einem anderen Staat. ${ }^{10}$ In vielen Bereichen gehört nicht nur die Berücksichtigung ausländischer Sachverhalte, sondern auch die Kooperation mit ausländischen Behörden zum Alltagsgeschäft der Verwaltung ${ }^{11}$ - Beispiel: Doppelbesteuerungsabkommen.

Für die rechtliche Bewältigung dieses Prozesses - hier eher untechnisch verstanden als Internationales Verwaltungsrecht ${ }^{12}$ - muss die Wis-

\footnotetext{
${ }^{8}$ Klassisch dazu W. Friedmann The Changing Structure of International Law, 1964. Aus (nicht nur) historischer Sicht bedeutsam G. Jellinek Die Lehre von den Staatenverbindungen, 1882, insbesondere $158 \mathrm{ff}$;; ferner $O$. Mayer Deutsches Verwaltungsrecht, Band 2, 1. Aufl. 1896, 459 ff.; der entsprechende Abschnitt ist in der 3. Aufl., 1924, nicht mehr enthalten. Zur heutigen Lage vor allem Tietje (Fn. 5), passim, ferner W. Wessels Die Öffnung des Staates 2000.

9 Ist eine Kündigung nicht ausdrücklich oder implizit vorgesehen, ist sie nur bei Wegfall der Geschäftsgrundlage oder in vergleichbar geregelten Fällen zulässig; siehe im einzelnen Art. 54 WVRK. Dazu etwa $M$. Vierheilig Die rechtliche Einordnung der von der Weltgesundheitsorganisation beschlossenen regulations, 1984, $21 \mathrm{ff}$.

10 Überschneidungen und Kombinationen werden damit nicht ausgeschlossen. So beruhen die Doppelbesteuerungsabkommen - Musterbeispiel einer horizontalen Öffnung des Staates - weltweit wesentlich auf einem Musterabkommen der OECD und damit einer vertikalen Internationalisierung; dazu $K$. Vogel in ders./M. Lehner (Hrsg.) Doppelbesteuerungsabkommen, 3. Aufl. 2003, Einleitung Rn. $35 \mathrm{ff.;}$ E. Reimer in: Möllers/ Voßkuhle/Walter (Fn. 5), 181 (187). Auch grenznachbarschaftliche Kooperationen (zu diesen noch bei Fn. 43, 153, 158 und 174) beruhen zum Teil parallel auf vertikaler und horizontaler Kooperation.

11 Hierzu als ältere Darstellung das enzyklopädische Werk von $K$. Neumayer Internationales Verwaltungsrecht, 4 Bände 1910-1936. Zur empirischen Lage aus heutiger Sicht Wessels (Fn. 8).

12 Die Überzeugungskraft dieser (hier vorgegebenen) Begriffsbildung muss offen bleiben (vgl. noch bei Fn. 198). Jedenfalls wird der Begriff „Internationales Verwaltungsrecht" unterschiedlich verwendet. Ähnlich wie hier M. Ruffert in W. Hoffmann-Riem/ E. Schmidt-Aßmann/A. Voßkuhle (Hrsg.), Grundlagen des Verwaltungsrechts I, 2006, $\$ 17 \mathrm{Rn}$. 150. Daneben wird er teils für das Recht internationaler Verwaltung, vor allem durch Internationale Organisationen (so etwa C.F. Amerasinghe ICLQ 45 [1996], 773 [774]; Friedmann [Fn. 8], 159 ff.; $K$. Vogel EPIL I, 1992, 22 [23]; vgl. auch Ruffert ebd.), teils - in Analogie zum Internationalen Privatrecht - zur Umschreibung des verwaltungsrechtlichen Kollisionsrechts genutzt (dazu C. Ohler Die Kollisionsordnung des Allgemeinen Verwaltungsrechts, 2005; G. Hoffmann Internationales Verwaltungsrecht,
} 
senschaft Rahmenbedingungen erarbeiten. Bei dem Versuch einer entsprechenden Systematisierung der Probleme werden nachfolgend vor allem inhaltliche Aspekte behandelt; Herr Biaggini wird dann stärker methodische Gesichtspunkte beleuchten. $\mathrm{Zu}$ beantworten sind insbesondere vier, aus dem rein nationalen Recht bekannte Fragen ${ }^{13}$ : wie steht es um die Legitimation des jeweiligen Handelns, wie um seine rechtsstaatliche Qualität? Wie wird Rechtsschutz gewährt, und wer haftet?

\section{II. „Vertikale“ Internationalisierung der Verwaltung}

\section{Empirische Ebene}

Die Zahl von Organisationen, die auf völkervertraglicher Grundlage bestehen und selbst Verwaltungsaufgaben erfüllen oder den nationalen Verwaltungen Vorgaben machen, liegt insgesamt bei rund 250.14 Hinzu kommen zahlreiche unterhalb dieser Ebene verfasste, faktisch aber in ähnlicher Weise wirkende Institutionen und Diskussionsforen neudeutsch Netzwerke. Genannt seien etwa die IOSCO, eine nach kanadischem Privatrecht verfasste Organisation von Behörden der Finanzdienstleistungsaufsicht ${ }^{15}$, oder der Baseler Ausschuss für Bankenaufsicht, ein informeller Zusammenschluss von Zentralbankpräsidenten ${ }^{16}$. Private wirken bei einigen Internationalen Organisationen mit, etwa bei

in: I. v. Münch [Hrsg.] Besonderes Verwaltungsrecht, 7. Aufl. 1985, 851 ff.). Siehe zu den verschiedenen Definitionen auch den 2. Bericht von G. Biaggini I 2 sowie E. Steindorff Verwaltungsrecht, Internationales, in: K. Strupp/H.-J. Schlochauer (Hrsg.) Wörterbuch des Völkerrechts, Band 3, 2. Aufl. 1962, 581. - Hier ausgeklammert bleibt die Internationalisierung des Verwaltungsrechts rein durch völkerrechtliche Verträge (dazu F. C. Mayer Die Internationalisierung des Verwaltungsrechts, im Erscheinen).

13 Vgl. auch Kingsbury/Krisch/Stewart Law and Contemporary Problems 68 (2005), 15 (43). Andere Autoren heben allein die verfahrensrechtlichen Probleme (Partizipation und Rechtsschutz) hervor: siehe etwa $S$. Cassese Global Administrative Law: An Introduction, verfügbar unter http://iilj.org/global-adlaw/documents/Cassesepaper.pdf, 27; C. Harlow EJIL 17 (2006), 187 (190f., 195, 204 ff.).

14 Das Yearbook of International Organizations 2004/05 gibt die Zahl 245 an, davon 34 universell angelegte Organisationen; alle informelle, aber von Hoheitsträgern getragenen Kooperationen eingeschlossen werden 1878 angegeben.

15 Dazu Möllers ZaöRV 65 (2005) 351 (362ff.).

${ }^{16}$ Mitglieder sind 13 Zentralbankpräsidenten wichtiger Industriestaaten. Dazu $A$. v. Aaken in: Möllers/Voßkuhle/Walter (Fn. 5), 217 (225ff.), sowie C. Ohler ebd., 257 (263); ausführlich M.S. Barr/G.P. Miller Global administrative law: the view from Basel, EJIL 17 (2006), $15 \mathrm{ff}$. 
der Internationalen Fernmeldeunion, ${ }^{17}$ und tragen Institutionen eigenständig. Als Beispiel hierfür sei die Internationale Standardisierungsorganisation ISO genannt. ${ }^{18}$

Internationale administrative Einzelfallentscheidungen, wie sie etwa im Rahmen des listing-Verfahrens des UN-Sicherheitsrates ergehen, sind allerdings empirisch eher selten. Außer bei der Personalverwaltung, die bei jeder Organisation $\mathrm{zu}$ finden ist, ${ }^{19}$ kommen diese vor allem im Rahmen territorialer Sonderregime vor: bei Flussregimen wie der Rheinschifffahrtskommission, 20 bei der im Seerechtsübereinkommen vorgesehenen Verwaltung der Schätze des Meeresbodens ${ }^{21}$ oder im Rahmen der Übernahme der Verwaltung eines Staates durch die UNO wie in Bosnien, dem Kosovo etc. ${ }^{22}$ Daneben gibt es eine internationale Verwaltung bei knappen Ressourcen: Weltbank und Währungsfonds ${ }^{23}$ vergeben Kredite an Staaten, die zudem ggf. an die Wahrung einer bestimmten Qualität des innerstaatlichen Verwaltungshandelns geknüpft sind $^{24}$; die Internationale Fernmeldeunion sorgt unter anderem für eine

${ }_{17}$ Dazu Tegge (Fn. 6), $92 \mathrm{ff} ., 123 \mathrm{ff}$. Gleiches gilt für die Codex alimentarius-Kommission (dazu noch Fn. 39); siehe C.H. Röhl in: Möllers/Voßkuhle/Walter (Fn. 5), 319 (330). Allgemein zur Rolle Privater bei der „Global Governance“ siehe G.F. Schuppert (Hrsg.), Global Governance and the Role of Non-State Actors, 2006. Die Beteiligung Privater an der Normsetzung ist aber - Beispiel DIN Normen (dazu BGHZ 139, 16 [19]) - auch auf nationaler Ebene bekannt; für eine Beachtlichkeit solcher Regelwerke für die staatliche Rechtsordnung ist jedoch ein entsprechender Rechtsanwendungsbefehl (im hier relevanten Kontext: bei Fn. 64f.) erforderlich. Ausführlich etwa J. Köndgen AcP 106 (2006), $477 \mathrm{ff}$.

${ }^{18}$ Dazu Kingsbury/Krisch/Stewart Law and Contemporary Problems 68 (2005), 15 (22f.); Röhl (Fn. 17), 322 ff.; K.T. Hallström in: Schuppert (Fn. 17), 81 ff.; zur Standardisierung in der Forstwirtschaft E. Meidinger EJIL 17 (2006), $47 \mathrm{ff}$.

${ }^{19}$ Dazu I. Seidl-Hohenveldern/H. Loibl Recht der Internationalen Organisationen, 7. Aufl. 2000, Rn. 1042 ff.; H.G. Schermers/N.M. Blokker International Institutional Law, 4. Aufl. 2003, $\$ 491 \mathrm{ff}$.

${ }^{20}$ Heutige Fassung: BGBl. 1969 II $598 \mathrm{ff}$.

21 Art. 153 ff. SRÜ, BGBl. 1994 II 1799; dazu etwa $R$. Wolfrum Die Internationalisierung staatsfreier Räume, 1984, $416 \mathrm{ff}$., zur Behörde $511 \mathrm{ff}$; ferner zur Antarktis etwa A. Kämmerer Die Antarktis in der Raum- und Umweltschutzordnung des Völkerrechts, 1994; zum Weltraum $S$. Hobe Die rechtlichen Rahmenbedingungen der wirtschaftlichen Nutzung des Weltraums, 1992, 257 ff.

22 Dazu M. Ruffert ICLQ 50 (2001), $613 \mathrm{ff}$; C. Stahn ZaöRV 51 (2001), $107 \mathrm{ff}$., mit Hinweisen auch auf historische Vorbilder (114ff.); speziell zu Bosnien-Herzegowina ferner A.M. Rehs Gerichtliche Kontrolle internationaler Verwaltung, 2006, $82 \mathrm{ff}$.; zum Kosovo T. Garcia RGDIP 104 (2000), $61 \mathrm{ff}$.

${ }^{23}$ BGB1. 1952 II 664 bzw. BGBl. 1978 II 13.

${ }^{24}$ Dazu P. Dann in: Möllers/Voßkuhle/Walter (Fn. 5), 5 (12ff.); zu entsprechenden "good governance"-Vorstellungen auch Kingsbury/Krisch/Stewart Law and Contemporary Problems 68 (2005), 15 (37). 
sachgerechte Aufteilung von Funkfrequenzen.25 Im Umweltbereich entscheidet die Internationale Walfangkommission über Fangmöglichkeiten. ${ }^{26}$

Über zahlreiche Politikfelder hinweg nehmen internationale Organisationen vor allem zwei Aufgaben wahr. Zunächst geht es um Gewinnung und Auswertung von Informationen. In vielen Bereichen Umweltschutz, Sicherheit, Menschenrechte - werden materielle Verpflichtungen der Staaten ergänzt durch solche, Berichte über jeweils erreichte Fortschritte zu verfassen;27 deren Auswertung kommt zum Teil einer Rechtsaufsicht nahe. ${ }^{28}$ Gelegentlich erfolgt die Überwachung auch in Form von Inspektionen. ${ }^{29}$ Benchmarking-Funktion haben etwa die im Rahmen der OECD durchgeführten, unter dem Namen PISA be-

\footnotetext{
${ }^{25}$ Art. 2 Abs. 2 lit. a, Art. 12 ITU-Konstitution (Fn. 6); Vollzugsordnung Funk, Kap. III. Dazu Tegge (Fn. 6), $171 \mathrm{ff}$.

${ }^{26}$ Zum entsprechenden Vertrag siehe BGBl. 1982 II 559. Rechtstechnisch geschieht dies durch Änderung des Anhangs zum Vertrag gemäß dessen Art. V; innerstaatlich wird diese durch eine Rechtsverordnung aufgrund Art. 2 Zustimmungsgesetz umgesetzt. Zu diesem Verfahren allgemein siehe in Fn. 68.

27 Allgemein dazu A. Chayes/A.Handler Chayes The new Sovereignty, 1995, $154 \mathrm{ff}$.; speziell zum Umweltschutz siehe konkret etwa Art. 7 Kyoto-Protokoll (BGBl. 2002 II 966; zu diesem ausführlich etwa $C$. Kreuter-Kirchhof Neue Kooperationsformen im Umweltrecht, 2005); allgemein $R$. Wolfrum RdC 1998, 9 (37); U. Beyerlin/T. Marauhn Rechtssetzung und Rechtsdurchsetzung im Umweltvölkerrecht nach der Rio-Konferenz 1992, 1997, 96ff.; U. Beyerlin Umweltvölkerrecht 2000, Rn. 481 ff.; zu den Menschenrechten etwa Art. 40 IPbürgR und dazu H.M. Empell, Die Kompetenzen des UNMenschenrechtsausschusses im Staatsberichtsverfahren, 1987; ferner Art. 16 IPwirtR und dazu A. Urmoneit Internationale Kontrolle mitgliedstaatlicher Verpflichtungen im Bereich des Sozialrechts, Diss. Bonn 1998, 31 ff.; $N$. Valticos FS Bernhardt, 645 (650ff.); zum Sozialrecht (Art. 19, 22 ILO-Konvention) Urmoneit ebd.

${ }^{28}$ Dazu näher Beyerlin/Marauhn (Fn. 27), 103. Siehe etwa die Nachfragemöglichkeit nach Art. XII Abs. 2 lit. d des Washingtoner Artenschutzübereinkommens, BGBl. 1975 II 777, und dazu Beyerlin (Fn. 27), Rn. 481, $489 \mathrm{ff}$.

${ }^{29}$ Allgemein dazu Chayes/Handler Chayes (Fn. 27), 174ff.; S. Oeter Netherlands Yearbook of International Law 28 (1997), 101 mit Darstellungen zur Abrüstung (110ff.), den Menschenrechten (130 ff.) und dem Umweltrecht (150 ff.). Siehe ferner etwa für den Bereich der Atomwaffen die Kontrollen durch die IAEA (BGBl. 1957 II 1358, insbesondere Art. XII) und dazu T. Lohmann Die rechtliche Struktur der Sicherungsmaßnahmen der Internationalen Atomenergieorganisation, 1993; für den Bereich der Chemiewaffen Art. VI, IX und X Chemiewaffenübereinkommen (BGBI. 1994 II 806) und dazu C. Bundscherer Deutschland und das Chemiewaffenübereinkommen 1997, $117 \mathrm{ff}$; für den konventionellen Bereich den KSE-Vertrag (BGBl. 1991 II 1154) und dazu C. Hoppe/ F. Rademacher EA 1991 225ff.; für den Umweltbereich allgemein Beyerlin/Marauhn (Fn. 27), 101 ff.; konkret etwa Art. XIII Abs. 2 des Washingtoner Artenschutzabkommens (Fn. 28); zur ILO Urmoneit (Fn. 27), 117.
} 
kannt gewordenen Tests im Schulbereich. ${ }^{30}$ In diesen Fällen tritt eine internationale Organisation zumindest mittelbar bzw. faktisch auch gegenüber Individuen auf. Sogar unmittelbar rechtliche Wirkung entfaltet die Weitergabe personenbezogener Informationen an internationale Institutionen wie etwa Interpol, den Zusammenschluss nationaler Polizeidienststellen. ${ }^{31}$

Weiterhin entwickeln internationale Organisationen einen beachtlichen Sachverstand auf vielen Gebieten ${ }^{32}$ und nutzen diesen zur Erarbeitung von Handlungsvorgaben für die Staaten, vor allem von verbindlichen oder zumindest faktisch wirkungsmächtigen - Standards ${ }^{33}$. Sie leisten so, auch wenn es zunächst nur um technische Probleme geht, auf internationaler Ebene bereichsspezifisch einen Beitrag zur Konkretisierung des Gemeinwohls, ${ }^{34}$ vielfach auch einen Ausgleich für die mit der Globalisierung verbundene Öffnung von Grenzen. Eine reiche, stärker verrechtlichte Tradition gibt es etwa bei der Kommunikation ${ }^{35}$, im Umweltschutz $^{36}$ sowie beim Gesundheitsschutz ${ }^{37}$, insbesondere beim

${ }^{30}$ Dazu OECD (Hrsg.) Lernen für die Welt von morgen, 2004; G.C. Ramm u.a. (Hrsg.) PISA 2003. Dokumente und Erhebungsinstrumente, 2006; E. Neuwirth u.a. (Hrsg.) PISA 2000 und PISA 2003, 2006; C. Langenfeld Die Verwaltung 40 (2007), $347 \mathrm{ff}$.

${ }^{31}$ Das Statut ist abgedruckt bei M. Baldus Polizeirecht des Bundes 2. Aufl. 2000 Nr. 40. Als internationale Organisation wird es angesehen von A. Randelzhofer FS Schlochauer, 1981, 531 (539ff.), mit dem Argument, dass die Polizeibehörden für ihren jeweiligen Staat handelten; aA Mokros in Denninger/Lisken (Hrsg.) Handbuch des Polizeirechts, 3. Aufl. 2001, Abschnitt O Rn 170 mit ausführlicher Darstellung von Interpol insgesamt Rn $168 \mathrm{ff}$. (die entsprechenden Ausführungen sind in der 4. Aufl., 2007, nicht mehr enthalten); dazu auch $G$. Stiebler Die Institutionalisierung der internationalen polizeilichen Zusammenarbeit auf dem Gebiet der Verbrechensvorbeugung und -bekämpfung in der „Internationalen Kriminalpolitischen Organisation“ Interpol (IKPO - INTERPOL), 1981.

${ }^{32}$ Zur immer wichtigeren Bedeutung von Wissen siehe in vorliegendem Zusammenhang M. Jachtenfuchs in: G. Hellmann/K.D. Wolf/M. Zürn (Hrsg.) Die neuen Internationalen Beziehungen, 2003, 495 (504f.).

${ }^{33}$ Dazu umfassend Röhl (Fn. 17), $319 \mathrm{ff}$,, sowie, vor allem zu Legitimationsfragen, $O$. Lepsius ebd., $345 \mathrm{ff}$.

${ }^{34}$ Tietje (Fn. 5), 610; J. Kokott Die Staatsrechtslehre und die Veränderung ihres Gegenstandes, VVDStRL 63, 7 (25). Ein Problem ergibt sich allerdings aus der nur begrenzten Kompetenz internationaler Institutionen, die es nicht immer gestattet, alle Aspekte eines Problems voll in den Blick zu nehmen.

${ }^{35}$ Siehe insbesondere den Weltpostverein (Fn. 7) und die Internationale Telekommunikations-Union (Fn. 6).

${ }^{36}$ Hier gibt es eine ganze Reihe von Organisationen und Strukturen. Siehe zum Kyoto-Protokoll bereits in Fn. 27; ferner allgemein $M$. Kilian Umweltschutz durch Internationale Organisationen, 1987; Tietje (Fn. 5), $367 \mathrm{ff}$.

${ }^{37} \mathrm{Zu}$ nennen ist vor allem die Weltgesundheitsorganisation WHO, BGBl. 1974 II 43; dazu Vierheilig (Fn. 9); Tietje (Fn. 5), $301 \mathrm{ff}$. 
Lebensmittelrecht ${ }^{38}$. So führte ein einschlägiger Beschluss der CodexAlimentarius-Kommission ${ }^{39}$ zur Verurteilung der EG im Streit mit den USA um hormonbelastetes Rindfleisch. ${ }^{40}$ Formalrechtlich weniger gehaltvoll, aber praktisch von nicht minderer Bedeutung ist diese Entwicklung im Bereich von Wirtschaft ${ }^{41}$ und Finanzen. Mit „Basel II“, das auch gestern angesprochen wurde, hat der erwähnte Ausschuss von Notenbankpräsidenten wichtige Vorgaben für die Eigenkapitalausstattung von Banken geschaffen. ${ }^{42}$ - Abschließend sei die - häufig privatrechtlich organisierte - grenzüberschreitende Kooperation bei der Daseinsvorsorge erwähnt. ${ }^{43}$

\section{Die Stellung der Staaten gegenüber internationaler Verwaltung}

Vor allem bei der Vergabe knapper Ressourcen und bei der Auswertung von Berichten stehen Staaten internationaler Verwaltung faktisch ähnlich wie Individuen nationaler Verwaltung gegenüber. Rechtsstaatliche Standards sind zuvörderst in den jeweiligen Regelwerken zu suchen, die aber oft nur begrenzt ergiebig sind. ${ }^{44}$ Für die Rechtsbeziehun-

$38 \mathrm{Zu}$ nennen ist vor allem die Organisation für Ernährung und Landwirtschaft FAO, BGBl. 1971 II $1033 \mathrm{ff}$.

${ }^{39}$ Diese wurde von WHO und FAO gemeinsam eingesetzt; dazu $R$ Merkle Der Codex Alimentarius der FAO und WHO, 1994; Tietje (Fn. 5), 304ff.; Röhl (Fn. 17), $329 \mathrm{ff}$.

${ }^{40}$ Der entsprechende Bericht des Apellate Body des DSB der WTO ist auszugsweise abgedruckt in EuZW 1998, $157 \mathrm{ff}$. Dazu etwa C.D. Classen UTR 49 (1999), $345 \mathrm{ff}$.

41 Dazu etwa N. Horn RabelsZ 44 (1980), 423 ff.; K. Hailbronner FS Schlochauer, 1981, $225 \mathrm{ff}$.

42 Dazu etwa G. Hofmann (Hrsg.) Auf dem Weg zu Basel II: Konzepte, Modelle, Meinungen, 2001; E. Brezski Rating: Basel II und die Folgen, 2004; $M$. Cluse Basel II Handbuch zur praktischen Umsetzung des neuen Bankenaufsichtsrechts, 2005.

43 Schmidt-Aßmann Der Staat 45 (2006), 315 (320); ausführlich $M$. Niedobitek Das Recht der grenzüberschreitenden Verträge, 2001, 64ff. Beispielhaft sei genannt die Pomerania, ein von drei privatrechtlich verfassten Vereinigungen kommunaler Gebietskörperschaften in Deutschland (östliche Landkreise von Mecklenburg-Vorpommern, nordöstliche Landkreise von Brandenburg), Polen (nordwestliche Landkreise) und Schweden (südliche Landkreise) getragener Zusammenschluss. - Zu Art. 24 Abs. 1a GG gibt es bisher keinen Anwendungsfall, so jedenfalls das Ergebnis einer im Mai 2007 durchgeführten Umfrage bei den Staatskanzleien aller in Betracht kommenden Bundesländer sowie bei den Bundesministerien des Äußeren und des Inneren. Theoretisch zu dieser Norm, aber auch ohne Benennung eines einzigen praktischen Beispieles ausführlich $M$. Kotzur Grenznachbarschaftliche Zusammenarbeit in Europa, 2004. Vgl. weiterhin bei Fn. 153 und 174.

${ }^{44}$ Anderes dürfte für die Kreditvergabe durch Weltbank oder UNDP im Bereich der Entwicklungszusammenarbeit gelten; dazu ausfuhrlich Dann (Fn. 24). 
gen zwischen den Staaten haben die Streitschlichtungsgremien der WTO zwar etliche allgemeine Rechtsprinzipien entwickelt. ${ }^{45}$ Eine Übernahme von aus dem nationalen Recht bekannten verwaltungsrechtlichen Grundsätzen in das Völkerrecht - als allgemeine Rechtsgrundsätze im Sinne von Art. 38 Abs. 1 lit. c IGH-Statut - ist aber dennoch kaum möglich.46 Trotz der gestiegenen inhaltlichen Bedeutung von Gemeinwohlgesichtspunkten im Völkerrecht ist dieses in seiner formalen Struktur bis heute Koordinationsrecht; die Staaten weisen im Verhältnis zu internationalen Organisationen ein rechtlich deutlich größeres Grewicht auf als Individuen zu ihrem Staat. ${ }^{47}$

Verbindliche Vorgaben im Sinne eines ius cogens gibt es daher hier nicht; 48 menschenrechtliche Garantien wie das Rechtsschutzgebot ${ }^{49}$ sind nicht anwendbar. Möglich ist es jedoch, Leitlinien insbesondere für die Handhabung bestehenden Verfahrensrechts zu entwickeln, vor allem, soweit diese - wie etwa ein Recht auf Anhörung - zur rechtlich tragfähigen Klärung von Streitfragen sachlich unverzichtbar sind..$^{50}$ Schließlich begründen rechtswidrige Entscheidungen unstreitig eine Haftung. Für die Stellung von Staaten in internationalen Organisationen gilt insgesamt Vergleichbares. ${ }^{51}$ Soweit überhaupt Anleihen beim natio-

${ }^{45}$ G.J. Göttsche Die Anwendung von Rechtsprinzipien in der Spruchpraxis der WTORechtsmittelinstanz, 2005, mit Hinweisen zu Diskriminierung (199ff.), Verhältnismäßigkeit (248ff,; dazu auch J. Neumann/E. Türk in: M. Nettesheim/G. Sander [Hrsg.] WTORecht und Globalisierung, 2003, $103 \mathrm{ff}$.) sowie Verfahrensgarantien (332 ff.); speziell zu letzteren auch die Entscheidung des DSB-Appellate Body im die USA betreffenden shrimps-Fall (ILM 1999, 118) mit Hinweis in § 180f. zu entsprechenden Möglichkeiten der Verfahrensgestaltung (Anhörung, begründete Entscheidung) des handelnden Staates zur Vermeidung einer Diskriminierung.

46 Tendenziell aA wohl Dann (Fn. 24), 20.

47 Dazu etwa C. Engel DB, VVDStRL 56 (1997), 137f. sowie oben bei Fn. 9.

48 Vgl. auch Schermers/Blokker (Fn. 19), § 1336.

49 Siehe aber zu panels im Bereich der Weltbank Dann (Fn. 24), 37; Cassese (Fn. 13), 28. Daneben sehen die Kreditverträge der Weltbank offenbar auch - praktisch aber bedeutungslose - Schiedsgerichtsklauseln vor; siehe Dann ebd., 36. Zu weiteren Kontrollmechanismen ders. AVR 44 (2006), $381 \mathrm{ff}$.

${ }^{50}$ Siehe etwa zur internationalen Erfüllungskontrolle bei Verträgen (dazu in der Sache bei Fn. 27f.) Beyerlin/Marauhn (Fn. 27), 105 ff.; zur Kontrolle von Berichten nach Art. 26ff. ILO-Satzung Urmoneit (Fn. 27), 96 ff., mit dem Hinweis, dass sich die Praxis konsequent um Konsistenz bemühe. Vgl. ferner den Hinweis auf Verhandlungspflichten im Urteil des IGH zum Streit um die Fischereirechte vor Island, ICJ-Reports 1974, 3 (Rn. $73 \mathrm{fr}$.). Zum „Entstehen internationaler Rechtsstaatlichkeit“ aus politikwissenschaftlicher Sicht siehe den gleichnamigen Beitrag von B. Zangl in: S. Leibfried/M. Zürn (Hrsg.) Transformation des Staates, 2006, $123 \mathrm{ff}$.

31 So wird denn in der großen Darstellung internationaler Organisationen von Schermers und Blokker (Fn. 19) mit Blick auf die Entscheidungsfindung die Vielfalt der Ge- 
nalen Recht möglich sind, sind diese zudem eher im Gesellschafts- als im Verwaltungsrecht zu suchen.

\section{Internationale Verwaltung und nationales Recht}

\section{a) Organisationsrecht}

Aus Sicht des nationalen Organisationsrechts wirft die Mitwirkung in einer internationalen Organisation oder einem informellen Zirkel Fragen auf. Im Regelfall ist sie Sache des zuständigen Ministeriums. Geht es um mehr als um unmittelbar technische Fragen, wird zum Problem, dass hier nur „Fachbruderschaften“" zusammenkommen. 52 Gesellschaftliche Herausforderungen sind jedoch letztlich nur durch Bewertung und Abwägung divergierender Interessen zu bewältigen. ${ }^{53}$ Auf rein nationaler Ebene wird dies institutionell dadurch abgesichert, dass zumindest Gesetzesinitiativen einen Beschluss der gesamten Bundesregierung verlangen. Für die internationale Kooperation sieht $\S 38$ Abs. 2 der Gemeinsamen Geschäftsordnung der Bundesregierung (GGO) immerhin bei „grundsätzlichen Fragen“ eine Pflicht zur Information des Auswärtigen Amtes vor. Man muss sich aber fragen, ob die traditionelle Eigen-

staltungsmöglichkeiten betont ( $\S 708 \mathrm{ff}$ ); allgemeine Rechtsgrundsätze dienen nur der Lückenfüllung (ebd., § 1336). Die von Möllers ZaöRV 65 (2005), 351 (384ff.) und ihm folgend Schmidt-Aßmann FS R. Schmidt, 2006, 149 (159) geforderte Entwicklung rechtlicher Prinzipien für die Arbeit vor allem informeller Gremien kann daher realistischerweise nur in der Form erfolgen, dass deren Beachtung zur Bedingung der Mitwirkung nationaler Stellen bzw. der Umsetzung auf nationaler Ebene (dazu sogleich) gemacht wird; für die Herausarbeitung entsprechender allgemeiner Rechtsgrundsätze mit der Folge eines Bestehens bindenden Völkerrechts fehlt es an der Grundlage. Der Verweis auf die Arbeit des DSB (dazu bei Fn. 45) trägt nicht, denn dort ging es bislang um nationale Standards, die dem Ziel der WTO, einem freien Welthandel, entgegenstehen; deswegen ist es aus WTO-Sicht plausibel, hier Grenzen zu ziehen. Internationale Standards sind hingegen aus WTO-Sicht ein Vorteil, weswegen man sich insoweit kaum vorstellen kann, dass das DSB mit gleicher Konsequenz Rechtsprinzipien entwickelt, mit denen diese Standards in Frage gestellt werden können. Zu internationalen Verfahrensstandards bei internationalen Konferenzen W. Graf Vitzthum FS Schlochauer, 1981, 739 ff.; $M$. Limpert Verfahren und Völkerrecht, 1985.

\$2 Ein paralleles Problem stellt sich bei dem Ministerrat der EG; der Versuch des Konvents, dieses Problem durch Einführung eines einheitlichen Gesetzgebungsrates zu beseitigen (Art. I-23 Vf-Entwurf 2003), ist von der Regierungskonferenz schon 2004 gekippt worden (Art. I-24 Vf-Entwurf 2004).

${ }^{33}$ Siehe als Beispiel die Diskussion um Standards im Bankenbereich (Eigenkapitalausstattung) und dazu Möllers ZaöRV 65 (2005), 351 (357ff. mwN). 
verantwortlichkeit der Ministerien nach Art. 65 S. 2 GG hier eine problemadäquate Regelung darstellt. ${ }^{54}$

Verfassungsrechtlich ist Außenpolitik Sache der Regierung. ${ }^{55}$ Dementsprechend stellt $\S 140$ TKG klar, dass internationale Aktivitäten der Bundesnetzagentur im Bereich der Telekommunikation ${ }^{56}$ im Auftrag der Bundesregierung stattfinden. Da es letztlich um die interne Organisation der Exekutive geht, besteht aber für auswärtiges Handeln von Behörden als solches kein Gesetzesvorbehalt; 57 $\$$ Abs. 3 Satz 2 des Gesetzes über den auswärtigen Dienst (GAD) setzt solches Handeln auch jenseits dieses Dienstes voraus. Soweit nach einigen Verträgen explizit nicht die Regierung, sondern Verwaltungen in die internationale Kooperation eingebunden sind, ist das sachlich vernünftig und rechtlich unbedenklich, soweit es um Eilmeldungen in Unglücksfällen ${ }^{58}$ oder wie beim internationalen Tierseuchenamt ${ }^{59}$ um unmittelbar technische Fragen geht.

\section{b) Innerstaatliche Bedeutung internationaler Standards}

Inhaltlich gesehen besteht die für den Bürger wie die nationale Verwaltung wichtigste Tätigkeit internationaler Organisationen in der Er-

\footnotetext{
${ }^{54} \mathrm{Im}$ internationalen Vergleich erweist sich die regierungsinterne Koordination der auswärtigen (einschließlich der europäischen) Beziehungen in Deutschland als besonders schlecht; siehe L. Metcalfe, IRAS/RISA 1994, 271 (285); König VA 92 (2001), 475 (501).

55 Möllers ZaöRV 65 (2005), 351 (372).

${ }^{56}$ Nach der Definition im Annex zur Satzung ist Verwaltung jede staatliche Dienststelle, die für die Maßnahmen zur Erfüllung der Verpflichtungen aus der ITU-Konstitution verantwortlich ist; dazu Tegge (Fn. 6), $90 \mathrm{ff}$.

57 So aber Möllers ZaöRV 65 (2005), 351 (375). Als immerhin gesetzlich geregeltes Beispiel siehe $\$ 342$ Abs. 1 S. 1 Nr. $3 \mathrm{HGB}$, wonach die Bundesregierung mit einer privaten Normierungsagentur einen Vertrag schließen kann, der diese unter anderem bevollmächtigt, die Bundesregierung bei einschlägigen internationalen Konferenzen zu vertreten. Diese Konstruktion geht auf US-amerikanisches Vorbild zurück ( $F$. Ebke MüKo HGB, Band 4, 2001, $\$ 342$ Rn. 3). Mittlerweile hat die Bundesregierung einen entsprechenden Vertrag mit dem „Deutschen Rechnungslegungs-Standards Committee e.V.“, Berlin, abgeschlossen. Dazu H. Merkt in: O. Baumbach/K.J. Hopt, HGB, 32. Aufl. 2006, § $342 \mathrm{Rn}$. 2. Zur einschlägigen politikwissenschaftlichen Forschung siehe etwa A. Nölke in: G. Hellmann/K.D. Wolf/M. Zürn (Fn. 32), $519 \mathrm{ff}$.

${ }_{S B} \mathrm{Zu}$ Meldungen in Unglücksfällen Art. 7 IAEO-Benachrichtigungsübereinkommen (BGBl. 1989 II 435); Art. 4 IAEO-Hilfeleistungsübereinkommen (BGBl. 1989 II 441); ähnlichen technischen Funktionen dient die Anlaufstelle nach Art. 5 des Basler Übereinkommens über die Kontrolle der grenzüberschreitenden Verbringung gefährlicher Abfäle und ihrer Entsorgung (BGBl. 1994 II 2704).

${ }^{59}$ BGBl. 1974 II 677 (Übereinkommen, dem die Satzung des Amtes als Anlage beigefügt ist). Nach Art. 4 der Satzung sind nur eigene Forschung, die Sammlung von Informationen sowie die Vorbereitung von Abkommen als Aufgaben vorgesehen.
} 
arbeitung von Standards. Diese Aufgabe steht wie auf der nationalen Ebene im Grenzbereich von Normsetzung und Verwaltung, verstanden als Normausführung. Rechtlich kann man bei völkerrechtlich verfassten internationalen Organisationen unterscheiden zwischen unmittelbar verbindlichen Beschlüssen, 60 Beschlüssen, deren Verbindlichkeit die Mitgliedstaaten durch eine Erklärung verhindern können, ${ }^{61}$ ratifikationsbedürftigen Beschlüssen ${ }^{62}$ und Empfehlungen. ${ }^{63}$ Beschlüsse nicht völkerrechtlich verfasster Gremien weisen ggf. politische, aber keine rechtliche Bindungswirkung auf.

${ }^{60}$ Etliche Verträge sehen (qualifizierte) Mehrheitsentscheidungen vor, so das Montrealer Protokoll über Stoffe, die zu einem Abbau der Ozonschicht führen (aktuelle Fassung: BGBl. 2003 II 346; siehe dort Art. 2 Abs. 9 lit. c).

61 Üblicherweise wird hier von der Möglichkeit eines „opt out" gesprochen; dazu Vierheilig (Fn. 9), 63 ff.; Aston (Fn. 7) 169 ff. Beispiele bilden etwa das Übereinkommen über die internationale Beförderung leicht verderblicher Lebensmittel (ATP-Übereinkommen, BGBl. 1974 II 565, dort Art. 18); das Übereinkommen zum Schutz der Meeresumwelt des Nordostatlantiks (BGBl. 1994 II 1360, dort Art. 19 Abs. 3); das Washingtoner Artenschutzübereinkommen (Fn. 28, dort Art. XV Abs. 1 und 3 betreffend die Anhänge zum Abkommen); das WHO-Abkommen (Fn. 37, dort Art. $21 \mathrm{f}$.); das Abkommen über die Internationale Zivilluftfahrt (Abkommen von Chicago, BGBl. 1956 II 411, dort Art. 38). Vielfach wird eine Nutzung dieser Möglichkeit zur Gewährleistung der innerstaatlichen Mitwirkungsrechte des Bundestages verlangt, wenn die fraglichen Maßnahmen nicht durch Rechtsverordnungen oder einfache VerwaltungsmaBnahmen umgesetzt werden können: Vierheilig ebd., 188; $O$. Rojahn in: I. v. Münch/ P. Kunig (Hrsg.) GG II, 4./5. Aufl. 2001, Art. 59 Rn. 45a; U. Fastenrath Kompetenzverteilung im Bereich der auswärtigen Gewalt, 1986, 230f.; aA I. Pernice in: H. Dreier (Hrsg.) GG II, 2. Aufl. 2006, Art. 59 Rn. 41; B. Kempen in: H. v. Mangoldt/ F. Klein/C. Starck (Hrsg.) GG II, 5. Aufl. 2005, Art. 59 Rn. 48, der eine antezipierte Zustimmung des Bundestages annimmt; in der Konstruktion überzeugender: Aston (Fn. 7), 197 f.: konsentierter Verzicht auf das Konsensprinzip (dem der Gesetzgeber zugestimmt hat). Nun gestattet Art. 24 Abs. 1 GG - weitergehend - sogar die Übertragung (wenn auch nur bestimmter) Hoheitsrechte (zu den Anforderungen: BVerfGE 58, 1 [36f.]; 68, 1 [98]); daher überzeugt die erwähnte Auffassung allenfalls, soweit die Änderungen nicht auf einen rechtlich hinreichend klar definierten Rahmen beschränkt sind, was in der Praxis aber regelmäßig der Fall ist. Für die Erteilung eines spezifischen innerstaatlichen Anwendungsbefehls ist jedoch ein Gesetz (oder eine Verordnung) notwendig; siehe bei Fn. 65 .

62 Siehe etwa das WHO-Abkommen (Fn. 37, dort Art. 19), ferner die Regeln des Codex alimentarius (dazu in Fn. 39); dazu Tietje (Fn. 5), 311; Merkle (Fn. 39), $38 \mathrm{ff}$.

${ }^{63}$ Siehe etwa die Abkommen über die Weltorganisation für Meteorologie WMO (BGBI. 1970 Il 19, dort Art. 8 lit. b) sowie die Welternährungsorganisation FAO (Fn. 38, dort Art. IV Abs. 3 und 4). 


\section{aa) Legitimationsbezogene Fragen}

Rechtsakte internationaler Organisationen außerhalb von Art. 24 GG richten sich grundsätzlich an das Völkerrechtssubjekt Bundesrepublik Deutschland. ${ }^{64}$ Rechte und Pflichten des einzelnen können nur durch einen eigenständigen Anwendungsbefehl begründet werden. ${ }^{65} \mathrm{Der} \mathrm{Hin}-$ weis auf die parlamentarische Zustimmung zum Vertrag über die entsprechende Organisation reicht nicht;66 anderenfalls läge faktisch eine

${ }^{64}$ Sieht man einmal von der (heute auf Art. 23 GG gestützten) Europäischen Gemeinschaft ab, bleibt die quantitative (dazu etwa C.D. Classen in: v. Mangoldt/Klein/ Starck [Fn. 61], Art. 24 Rn. 59 ff.) deutlich hinter der qualitativen Dimension von Art. 24 GG (dazu etwa Wahl [Fn. 5] 20: „Verwandlung des Staatsverständnisses“) zurück (zu dieser Diskrepanz Wahl ebd. 25). - Im Anwendungsbereich des europäischen Gemeinschaftsrechts erfolgt die Internationalisierung des Verwaltungsrechts häufig über dieses; dazu etwa Tietje (Fn. 5), 312, $443 \mathrm{ff}$; $A$. Hatje Halle-Vortrag ++, für das Lebensmittelrecht Merkle (Fn. 39), 43 ff., 53 ff.; für das Umweltrecht Durner in: Möllers/Voßkuhle/ Walter (Fn. 5), 121 (132). Da der EuGH das Völkerrecht grundsätzlich als Bestandteil des Gemeinschaftsrechts ansieht, allerdings im Einzelfall prüft, ob tatsächlich unmittelbare Wirkungen, insbesondere im Sinne von Rechten und Pflichten für den Einzelnen begründet werden (etwa Slg. EuGH, Rs. C-280/93 - Deutschland/Rat, Slg. 1994, I-4973 Rn. $105 \mathrm{ff}$.), stellen sich die Probleme dort auf der formalen Ebene (Art der Umsetzung) zum Teil anders dar als im deutschen Recht; die legitimationsbezogenen Probleme sind hingegen ähnlich.

65 P. Kunig in: W. Graf Vitzthum (Hrsg.) Völkerrecht, 4. Aufl. 2007, II Rn. 165; Tietje (Fn. 5), 589; P. Rösgen Rechtssetzungsakte der Vereinten Nationen und ihrer Sonderorganisationen, Diss. Bonn 1985, $246 \mathrm{ff}$. Dies gilt auch, wenn das bestehende innerstaatliche Recht völkerrechtlichen Vorgaben entspricht („Parallelabkommen“); siehe dazu Fastenrath (Fn. 61), 223; Pernice (Fn. 61), Art. 59 Rn. 35; Kempen (Fn. 61), Art. 59 Rn. 71; tendenziell aA für das geltende Recht $A$. v. Bogdandy/D. Zacharias NVwZ 2007, 527 (530). In anderen Staaten sind verbindliche Beschlüsse internationaler Organisationen automatisch Teil der innerstaatlichen Rechtsordnung, so in Belgien (dazu J. Verhoeven in: P.M. Eisemann [Hrsg.] Lintégration du droit international et communautaire dans l'ordre juridique international. Étude de la pratique en Europe, 1996, 115 [140]), Frankreich (Art. 55 Vf. und dazu E. Decaux u.a. ebd., 241 [270f.]), Luxemburg (dazu R. Biever u.a. ebd., 407 [423]), den Niederlanden (Art. 93 Vf. und dazu C.M. Brölmann/E.W. Vierdag, ebd., 433 [452]) und Spanien (Art. 96 Vf. und dazu R. Bermejo Garcia u. a. ebd., 183 [221 f.]); zusammenfassend $E$. Roucounas ebd. 39 ( 41 f.).

${ }^{66}$ So aber wohl BVerfGE 104, 151 (209); EuGRZ 2007, 331 (335). Tendenziell gegensätzlich dazu, aber wie im Text BVerfGE 111, 307 (324ff.): Urteile des EGMR sind zwar von den innerstaatlichen Stellen bei der Auslegung und Anwendung des innerstaatlich geltenden Rechts zu berücksichtigen; eine unmittelbare Wirkung aber wird nicht angenommen. Vgl. auch BVerfGE 74, 358 (370); instruktiv ferner BVerfG (K), EuGRZ 2007, 467 (470f.). Entsprechendes gilt etwa für verbindliche Auslegungsentscheidungen nach Art. XXIX lit. $a$ und b IWF- und Art. IX lit. a und b Weltbankabkommen (Fn. 23; zur Praxis, eher kritisch, $R$. Schmidt Der Verfassungsstaat im Geflecht der internationalen Beziehungen, VVDStR L 36 [1977], 65 [81]) und würde, wenn sich nicht praktisch alles im Rahmen des Gemeinschaftsrechts abspielen würde, auch für Entscheidung des DSB 
Übertragung von Hoheitsrechten vor. Dieser Anwendungsbefehl kann durch den Gesetzgeber ${ }^{67}$ oder - nach Art. 80 GG - von der Exekutive ${ }^{68}$

der WTO gelten (naturgemäß nur mit dem entsprechenden Inhalt, der im zuletzt genannten Fall letztlich doch begrenzt ist, worauf der EuGH zu Recht hingewiesen hat; siehe Fn. 64).

${ }^{67}$ Unklar ist, ob hier Art. 59 Abs. 2 GG (analog) Anwendung finden kann, wie dies in der Staatspraxis geschieht (siehe etwa die Zustimmungsgesetze zu „regulations“ der WHO: BGBl. 1955 II 1060; 1965 II 1413) und in der Literatur angenommen wird: Rojahn (Fn. 61), Art. 59 Rn. 45a; C. Engel Völkerrecht als Tatbestandsmerkmal deutscher Normen, 1989, 32; Vierheilig (Fn. 9), 187. Ein Ziel der Norm, die Vermeidung völkerrechtlicher Bindung ohne parlamentarische Zustimmung, kann aber in Fällen zuvor bereits eingetretener völkerrechtlicher Verbindlichkeit gar nicht mehr erreicht werden. Die Aussage, dass den auf internationaler Ebene vorgenommenen Änderungen „zugestimmt" werde (so Art. 1 des soeben erwähnten Zustimmungsgesetzes BGBl 1965 II 1413), geht daher ins Leere. Im ersten Fall, 1955, wurde sprachlich und inhaltlich schief sogar vom „Beitritt“ zu den Vorschriften gesprochen; richtig demgegenüber Art. 1 der Verordnung $\mathrm{zu}$ einer zusätzlichen WHO-regulation, BGBI. 66 II 802, wonach die Änderungen (zu ergänzen: innerstaatlich) „in Kraft gesetzt“ werden. Der im Zustimmungsgesetz üblicherweise enthaltene Anwendungsbefehl für den völkerrechtlichen Vertrag im innerstaatlichen Rechtsraum als Völkerrecht (mit der Folge, dass völkerrechtliche, nicht wie bei einer Parallelgesetzgebung innerstaatliche Auslegungsregeln Anwendung finden [dazu etwa $J$. Masing FS Böckenförde, 1995, 51]) wiederum kann durch entsprechenden (in der Regel statischen, dazu bei Fn. 70) Verweis in einem normalen Bundesgesetz erteilt werden ( $P$. Rösgen [Fn. 65], 259). Dies spricht gegen die analoge Anwendung von Art. 59 Abs. 2 GG in Fällen bereits eingetretener Bindung. So kommt diese Norm nach hier vertretener Auffassung nur in Betracht, wenn (zunächst) ein opt-out (dazu Fn. 61) praktiziert wurde.

68 Zum Teil sind die einschlägigen Verordnungsermächtigungen zu pauschal und damit verfassungswidrig. Siehe zu § 6a WHG $R$. Breuer ZfW 1999, 227 ff.; M. Kotulla Wasserhaushaltsgesetz, 2003, §6a Rn. 26;K. Czychowski/M. Reinhardt Wasserhaushaltsgesetz, 8. Aufl. 2003, $\S$ 6a Rn. 5); zur Geltung von Art. 80 GG auch in internationalen Zusammenhängen siehe ferner BVerfG (K) EuGRZ 2007, 231 (233). Anders ist die Lage, wenn sich die Ermächtigung auf die Durchführung bindender Beschlüsse einer bestimmten internationalen Organisation bezieht, wie in einigen einschlägigen Zustimmungsgesetzen der Fall; siehe etwa Art. 2 des Gesetzes zum ATP-Übereinkommen (Fn. 61); Art. 2 des Gesetzes zum Washingtoner Artenschutzabkommen (dazu Fn. 28), BGBl. 1975 II 773 („Änderungen ..., die sich im Rahmen der Ziele des Abkommens halten"); ebenso Art. 2 des Zustimmungsgesetzes zu den internationalen Übereinkommen zum Schutz der Meeresumwelt des Ostseegebietes und des Nordostatlantiks (BGBl. 1994 II 1355). In solchen Fällen können nämlich die einschlägigen vertraglichen Kompetenzbestimmungen zur Konkretisierung herangezogen werden. Deren Auslegung ist allerdings nicht immer unproblematisch. Als Negativbeispiel mag die Entwicklung bei der UNESCO dienen, die zu einem vorübergehenden „Rückzug“ der USA geführt hat (dazu M. Flory AFDI 1985, 653 [666ff.]). Demgegenüber hat der IGH in seinem Gutachten zur Rechtmäßigkeit von Atomwaffen die Grenzen der Kompetenzen der WHO deutlich unterstrichen (ICJ Reports 1996, $66 \$ 20$ ff.): diese dürfe sich nur 
erteilt werden. Er kann insbesondere in Form eines statischen Verweises erfolgen, wie er in einer jüngst ergangenen Kammerentscheidung des BVerfG zu international festgesetzten Altersgrenzen von Piloten eine Rolle spielte. ${ }^{69}$ In den vom BVerfG akzeptierten engen Grenzen ${ }^{70}$ ist aber auch ein dynamischer Verweis möglich. In deren Lichte dürfte etwa die arzneimittelrechtliche Verpflichtung, bei der Bezeichnung von Inhaltsstoffen von Arzneimitteln die internationalen Kurzbezeichnungen der Weltgesundheitsorganisation (WHO) zu verwenden, ${ }^{71}$ unproblematisch sein. ${ }^{72}$ Ein solcher Anwendungsbefehl kann schließlich auch internationale Empfehlungen innerstaatlich zu verbindlichen Vorgaben machen. So kann nach $\S 5$ Abs. 2 BtMG die Erteilung der nach $\S 3$ BtMG für Anbau und Herstellung von sowie Handel mit Betäubungsmitteln erforderlichen Erlaubnis unter anderem verweigert werden, wenn sie „Beschlüssen, Anordnungen oder Empfehlungen zwischenstaatlicher Einrichtungen der Suchtstoffkontrolle entgegensteht“. Auch ohne einen solchen speziellen Anwendungsbefehl aber besteht die Verpflichtung zur völkerrechtskonformen Auslegung des nationalen Rechts. ${ }^{73}$ Verbindliche internationale Sekundärrechtsakte sind daher im Rahmen

mit den Wirkungen, nicht aber mit der Rechtmäßigkeit des Einsatzes von Atomwaffen befassen ( $(21)$; daran ändere auch das ,implied-power“-Prinzip nichts (\$ 25); dazu $M$. Ruffert AVR 38 (2000), $129 \mathrm{ff}$.

${ }^{69}$ BVerfG (K), EuGRZ 2007, 231 (232f.) unter Bezugnahme auf einen Beschluss der Joint Aviation Authorities, ein assoziiertes Organ der European Civil Aviation Conference; dazu Tietje (Fn. 5), 458f. Siehe ferner etwa $\$ 32$ Abs. 3 Tierschutztransportverordnung, BGBI. 1999 I 1337.

70 Siehe etwa BVerfGE 26, 338 (365ff); 78, 32 (35f.); H. Schulze-Fielitz in: Dreier (Fn. 61), Art. 20 R Rn. 144; tendenziell ablehnend dagegen Dreier ebd., Art. 20 D Rn. 122f.; R. Poscher in diesem Band, B II 2.

${ }^{71} \S 10$ Abs. 6 Nr. 1 AMG.

$72 \S 292 \mathrm{a}$ HGB a.F. zur Bilanzierung deutscher Unternehmen nach ,international anerkannten Rechnungslegungsgrundsätzen" (statt nach deutschen) wird zum Teil als eine (unzulässige) dynamische Verweisung angesehen, stellt aber eine (zulässige) Option zur Rechtswahl dar. Dazu bei Fn. 154.

${ }^{73}$ Dazu schon in Fn. 66; ferner C. Tomuschat HStR VII $\$ 172$ Rn. 35; zum völkerrechtlichen Umweltrecht Durner (Fn. 64), 144. Diese Verpflichtung besteht jedoch nicht, soweit es völlig an einem (nach außen wirkenden) innerstaatlichen Rechtsakt fehlt wie etwa im Fall des Übereinkommens zum Schutz des Kultur- und Naturerbes der Welt vom 23. 11. 1972 (BGBl. 1977 II 213), dem die Bundesrepublik nur durch Regierungsbeschluss beigetreten ist. AA insoweit $v$. Bogdandy/Zacharias NVwZ 2007, 527 (531 f.); offen lassend BVerfG (K), EuGRZ 2007, 355 (358). Vielmehr kann die Konvention innerstaatlich nur die Wirkung einer Verwaltungsvorschrift entfalten. $\mathrm{Zu}$ anderenfalls ggf. drohenden Konflikten mit dem Gebot effektiven Rechtsschutzes siehe bei Fn. $161 \mathrm{f}$. 
der Auslegung und Anwendung des innerstaatlichen Rechts heranzuziehen. ${ }^{74}$

Neben völkerrechtlich verbindlichen Beschlüssen stehen Empfehlungen internationaler Organisationen sowie sonstige Absprachen und damit Instrumente spezifisch administrativen Charakters. Ob und inwieweit diese von einer nationalen Verwaltung bei der Nutzung einschlägiger Ermessens-, ggf. aber auch Beurteilungsspielräume herangezogen werden können oder sogar müssen, ist wissenschaftlich kaum geklärt. In der Sache hängt dies vom jeweiligen institutionellen Rahmen, der Art des Zustandekommens und der inhaltlichen Qualität des Standards ab. ${ }^{75}$ Bei der Erteilung der Erlaubnis für das Einleiten von Abwasser nach $\$ 7$ a WHG, wonach die Schadstofffracht des Abwassers so gering wie nach dem Stand der Technik jeweils möglich gehalten werden muss, können also internationale Vorgaben jeglicher Art berücksichtigt werden. ${ }^{76}$

Die insoweit unter Hinweis auf das Demokratieprinzip erhobene Kritik $^{77}$ trägt nicht; Herr Poscher hat das schon gezeigt. Abgesehen davon, dass die hier angesprochenen Sachfragen auch national in aller Regel allein durch die Exekutive geregelt werden, verlangt jeder Beitritt zu einer internationalen Organisation mit Beschlusskompetenz im Gegensatz jedenfalls zur Staatspraxis der fünfziger Jahre eine parlamentarische $\mathrm{Zu}$ stimmung. Soweit nicht Art. 24 Abs. 1 GG einschlägig ist, folgt dies aus Art. 59 Abs. 2 GG. $^{78}$ Damit wird internationale Beschlussfassung prin-

\footnotetext{
${ }^{74}$ Rojahn (Fn. 61), Rn. 45b; Rösgen (Fn. 65), $271 \mathrm{ff}$.

${ }^{75}$ K.M. Meessen NJW 1981, 1131 (1132); P. Kunig FS Doehring 529 (544ff.); vgl. auch Hailbronner (Fn. 41), 352 ff.; J.A. Frowein ZaöRV 36 (1976), 147 (165ff.).

${ }^{76}$ Dazu Tietje (Fn. 5), 421; U. Dieckert Die Bedeutung unverbindlicher Entschließungen internationaler Organisationen für das innerstaatliche Recht der Bundesrepublik Deutschland, 1993, 105. - Siehe ferner etwa zur polizeilichen Generalklausel VG Frankfurt/M, NJW 1988, 3032, mit Kritik bei W. Heusel NJW 1989, 2174; zu §§ 33 f. LMBG Tietje (Fn. 5), 339. Entsprechendes gilt für Verordnungsermächtigungen; siehe etwa § 56 LuftBO (Betriebsordnung für Lufffahrtgerät, BGBl. 1970 I 262); zur Umsetzung der WHO-regulations Vierheilig (Fn. 9), $161 \mathrm{ff}$. Weitere Beispiele bei Dieckert ebd., $102 \mathrm{ff}$.

77 Vgl. Schmidt (Fn. 66), 80; Engel (Fn. 68), 253 ff., 46 ff. Das BVerfG (K, EuGRZ 2007, 231 [233]) stellt in einem solchen Fall allein darauf ab, dass bei der Entscheidungsvorbereitung Art. $20 \mathrm{GG}$ gar nicht maßstäblich sei. Dieses Argument ist aus der Rechtsprechung zwar vertraut (vgl. BVerfGE 47, 253 [273]; 83, 60 [73]; 93, 37 [68]), aber dennoch in seiner Pauschalität zweifelhaft (dazu Röhl [Fn. 17], 342; Voßkuhle HStR III, 3. Aufl. 2005, §43 Rn. 22).

${ }^{78}$ Spätere Beschlüsse, deren Umsetzung inhaltlich dem Gesetzesvorbehalt unterfallen, können nämlich nicht ausgeschlossen werden. Dass diese ggf. noch einer Annahme durch die Mitgliedstaaten bedürfen, ändert wegen der politischen Vorwirkung der Entscheidung auf internationaler Ebene und der fehlenden Möglichkeit, einmal gefasste Be-
} 
zipiell auch verfassungsrechtlich legitimiert. ${ }^{79}$ Vor allem aber kann das geforderte "hinreichende Legitimationsniveau " ${ }^{\text {"80 }}$ nicht ohne Blick auf andere Verfassungsbestimmungen definiert werden, die es als „rechtlich verarbeitete politische Wirklichkeit" ${ }^{* 11}$ nicht erforderlich machen, daneben auch auf die Lebenswirklichkeit im Sinne eines „realistischen Demokratieverständnisses ${ }^{\text {" } 82}$ zurückzugreifen. Indem die Gesetze nach Art. 77 GG vom Bundestag „beschlossen“ werden, weist das Grundgesetz diesem zwar insoweit die Primärverantwortlichkeit zu. Demgegenüber fordert Art. 59 Abs. 2 GG die „Zustimmung“ des Bundestages zu den von der Regierung ausgehandelten Verträgen; damit liegt ganz formal betrachtet die Primärverantwortlichkeit hier bei der Exekutive. ${ }^{83}$

schlüsse noch zu ändern, nichts. Im Regelfall ist auch die Stellung der Bundesrepublik in der Staatengemeinschaft und sind damit die politischen Beziehungen der Bundesrepublik Deutschland (zu dieser Definition BVerfGE 1, 372 [381]) betroffen. Mit Hinweis auf das zweitgenannte Argument ebenso Schmidl-ABmann FS Schmidt (Fn. 5), 156; Tomuschat (Fn. 73) Rn. 39; Kunig (Fn. 65), II Rn. 162; tendenziell auch $R$. Wolfrum Kontrolle der auswärtigen Gewalt, VVDStRL 56 (1997), 38 (51); aA Tietje (Fn. 5), 541. Soweit der Beitritt zu einer internationalen Organisation allein nach einem Regierungsbeschluss erfolgte - etwa zur Weltgesundheitsorganisation 1951 (vgl. Bekanntmachung: BGBl. 1974 II 43), zur Welternährungs- und Landwirtschaftsorganisation 1950 (vgl. BGBl. 1971 II 1033) und zur UNESCO 1951 (vgl. BGBl. 1971 II 471) - war dies daher verfassungswidrig; aus heutiger Sicht kann die demokratische Legitimation nur, aber immerhin durch die lange Zeit der Akzeptanz dieser Mitgliedschaft begründet werden. Änderungen eines Abkommens kann hingegen auch durch Rechtsverordnung der Anwendungsbefehl erteilt werden, wenn das Zustimmungsgesetz die nach Art. 80 GG erforderlichen inhaltlichen Maßgaben enthält. Siehe dazu bei Fn. 68.

79 Vgl. auch Fn. $64 \mathrm{f}$.

${ }^{80}$ Dazu allgemein BVerfGE 83, 66 (72, 81); 89, 155 (182); 93, 37 (66f.); 107, 59 (87).

${ }^{81}$ Dazu C. Hillgruber in diesem Band, I 1.

82 So aber Poscher (Fn. 70), Leitsatz 15 und $178 \mathrm{ff}$.

${ }^{83} \mathrm{Vgl}$. auch C. Tomuschat Der Verfassungsstaat im Geflecht der internationalen Beziehungen, VVDStRL 36 (1977), 8 (27). Mit dem Völkergewohnheitsrecht nach Art. 25 GG gibt es sogar - wenn auch im Rang unter der Verfassung stehend - Bundesrecht, das über gar keine demokratische Legitimation verfügt. Eine Verfassungsänderung mit dem Ziel, Gleiches auch für das Vertragsrecht vorzusehen, verstieße nicht gegen Art. 79 Abs. $3 \mathrm{GG}$; in anderen demokratischen Staaten ist dies ohnehin üblich. Siehe zu den Niederlanden Art. 93 Vf. und dazu Brölmann/Vierdag (Fn. 65), 443f. Ähnlich ist die Rechtslage in Frankreich (Art. 55 Vf. und dazu Decaux u.a. [Fn. 65; 257 f.]) und Spanien (Art. 96 Vf. und dazu Bermejo Garcia u.a. [Fn. 65; 207 f.]), wo für eine innerstaatliche Anwendbarkeit jeweils nur eine (innerstaatliche) Publikation des Vertrages verlangt wird. Wenn das BVerfG in seinem Urteil zum Europäischen Haftbefehl (E 113, 273 [301]) anführt, dass demokratische Legitimation ggf. auch durch die Verweigerung der Umsetzung von völkerrechtlichen Verpflichtungen hergestellt werden könne, greift dies daher etwas kurz. 
Wichtiger noch sind inhaltliche Gesichtspunkte, die auch bei internationaler Kooperation unterhalb der völkerrechtlichen Schwelle Bedeutung entfalten. Diese trägt nämlich zur Sicherung des Friedens bei, dem nach der Präambel des Grundgesetzes das deutsche Volk „als gleichberechtigtes Glied ... dienen" will. ${ }^{84}$ Für den einzelnen verbessert sie die Rechtssicherheit und so die Möglichkeit zur Entfaltung von Freiheit über die Grenze hinweg. ${ }^{85}$ Das Beispiel der Justiz schließlich zeigt, dass die besondere Qualität des Entscheidungsverfahrens Abstriche beim Niveau der demokratischen Legitimation rechtfertigt. ${ }^{86}$ Vorliegend wiederum erschließt die internationale Kooperation einen Erfahrungsschatz, der vielfach - rechtsstaatlich wichtig - der inhaltlichen Qualität einer Entscheidung zugute kommt. ${ }^{87}$

Demokratische Sicherungswirkung können international - im Internet-Zeitalter leicht zu realisierende - Bemühungen um Transparenz entfalten, die ggf. auch Dritten, etwa den sogenannten „Nicht-Regierungsorganisationen", eine Beteiligung an einer Diskussion ermöglichen, ${ }^{88}$ wie dies gerade auch mit Blick auf die gestern diskutierten, oben bereits

${ }^{84} \mathrm{Vgl}$. auch Tomuschat (Fn. 83), 18; V. Röben Außenverfassungsrecht, 2007, $185 \mathrm{ff}$. Demgegenüber ordnet $F$. Schorkopf Grundgesetz und Überstaatlichkeit, 2007, $255 \mathrm{ff}$,, partikulare Handlungsoptionen als Element internationaler Gewaltenbalance ein. Da diese nur unter Bruch des Rechts zu erreichen ist, überzeugt dies nicht.

85 Stoll DVBl. 2007, 1064 (1069); Ohler ebd., 1083 (1086); zum erstgenannten Punkt auch B VerfGE 72, 66 (78f.). Ansprüche gegen ausländische Staaten sind nämlich im Regelfall schwer durchzusetzen: Im Inland steht die Immunität entgegen; in der ausländischen Rechtsordnung ist in zwischenstaatlich streitigen Angelegenheiten (wegen des dort geltenden nationalen Rechts) häufig nicht viel zu erreichen.

${ }^{86}$ Zum Problem A. Voßkuhle/G. Sydow JZ 2002, $673 \mathrm{ff}$.

87 Vgl. auch K.-H. Ladeur in: Möllers/VoBkuhle/Walter (Fn. 5), 375 (389); Tietje (Fn. 5), 625.

88 Dazu Harlow EJIL 17 (2006), 187 (199f.); mit Blick auf die ITU Tegge (Fn. 6) 97f.; vgl. auch Göttsche (Fn. 45), 233 ff.; T. Vesting Die Staatsrechtslehre und die Veränderung ihres Gegenstandes, VVDStRL 63, 42 (56f.); E. Riedel DB, VVDStRL 62 (2003), 108, mit berechtigter Kritik an $M$. Herdegen Informalisierung und Entparlamentarisierung politischer Entscheidungen als Gefährdungen der Verfassung?, ebd., 7 (13). - Eine Parlamentarisierung internationaler Organisationen, wie sie vor allem europäische Institutionen kennen (EG, Europarat, WEU, aber auch die NATO; dazu Schorkopf [Fn. 84], 192), wirft nicht nur die Frage danach auf, ob über-/international überhaupt eine sinnvolle Repräsentation möglich ist. Vor allem kann die zentrale Parlamentsaufgabe der Zusammenführung und Integration heterogener Willen im Rahmen spezialisierter internationaler Organisationen durch entsprechende „Partikularparlamente“ kaum bewältigt werden. So hat denn auch allenfalls die parlamentarische Versammlung des - mit vergleichsweise breiten Kompetenzen ausgestatteten - Europarates ein gewisses eigenständiges Profil entwickelt. 
erwähnten Baseler Standards der Fall gewesen ist. ${ }^{89}$ Auf nationaler Ebene ist - trotz aller gestern geäußerten Bedenken - eine Pflicht der Regierung zur Vorabinformation des Bundestages mit verfassungsrechtlicher Grundlage in der Organtreue anzunehmen ${ }^{90}$ - die ggf. auch bei nur informellen Absprachen besteht.

\section{bb) Rechtsstaatliche Fragen}

Eine Publikation der jeweiligen Standards ist unproblematisch über das Internet möglich. Können von allen Adressaten entsprechende Kenntnisse des Englischen verlangt werden - z. B. bei Piloten -, kann die Veröffentlichung auch durch Verweis auf einen in dieser Sprache verfassten Text erfolgen.91

Einer - inzident möglichen - Nachprüfung internationaler Beschlüsse durch nationale Gerichte sind mehrere Grenzen gesetzt. Nach Völkerrecht kommt bei der Auslegung von Verträgen auch der entsprechenden Praxis Bedeutung zu; ${ }^{92}$ dies begrenzt die Interpretationsfreiheit der Justiz. Ein Gericht kann also nicht ohne weiteres etwa unter Hinweis auf die vermeintliche Missachtung bestimmter internationaler Verfahrensregeln einen Standard für rechtswidrig erklären, wenn deren Handhabung regelmäßiger Praxis entsprach. ${ }^{93}$

Vor allem gilt dies für eine inhaltliche Kontrolle. Schon mit Blick auf nationale Standards, die unter Beteiligung entsprechender Experten erarbeitet worden sind, ist anerkannt, dass sie von Gerichten mangels eigener technischer Beurteilungskompetenz nur in Grenzen in Frage ge-

${ }^{89}$ Dazu speziell v. Aaken (Fn. 16), 248; Barr/Miller. EJIL 17 (2006), 15 (24ff.); $B$. Spenger in: Schuppert (Fn. 17), 101 (107ff.).

${ }^{90}$ T. Stoll DVB1. 2007, 1064 (1072); Kokott (Fn. 34), 33 f.; C. Tomuschat DB, VVDStRL 56 (1997), 114 (115); vgl. auch dens. (Fn. 83), 29; Poscher(Fn. 70), 186 ff. Der Hinweis auf die Mängel bei der Handhabung von Art. 23 Abs. 2 und 3 ist berechtigt, doch sind diese, wie ein Blick auf andere nationale Parlamente zeigt, in - behebbaren Mängeln in der Organisation der entsprechenden Arbeitsabläufe des Bundestages begründet, nicht im Wesen entsprechender Beteiligungsrechte. Dazu Classen (Fn. 64), Art. 23 Rn. 64 Fn. 1. Auch das von Röben (Fn. 84), 100, angesprochene parlamentarische Verhandlungsmandat setzt eine solche vorherige Information voraus.

91 Siehe etwa OVG Lüneburg, OVGE 44, 365 (369), vgl. auch BGHZ 102, 118 (123f.).

92 Art. 31 Abs. 3 lit. b WVRK. Dazu, speziell im Kontext internationaler Organisationen, C.F. Amarasinghe Principles of the Institutional Law of International Organisations, 2. Aufl. 2005, $49 \mathrm{ff}$.

${ }^{93}$ Liegt ein spezifischer nationaler Anwendungsbefehl vor, ist zudem zu prüfen, ob die Frage nach Verfahrensfehlern auf internationaler Ebene nicht ohnehin abgeschnitten ist. 
stellt werden können. ${ }^{94}$ Hier gilt das erst recht: welchem gerichtlich bestellten Sachverständigen kann man zutrauen, ein Problem besser im Blick zu haben als der geballte internationale Sachverstand, der ggf. auch gemeinsam mit der Politik die von Experten nicht zu bewältigenden Abwägungsfragen diskutiert und entschieden hat?95 Ist der Standard völkerrechtlich bindend, sind zudem die vom BVerfG für die innerstaatliche Wirkung von Urteilen des EGMR postulierten Grundsätze auch hier heranzuziehen: sie sind von den Gerichten zu berücksichtigen, das heißt im Regelfall verbindlich, und Abweichungen sind besonders zu begründen. ${ }^{96}$

\section{Individualbezogene Maßnahmen internationaler Verwaltung \\ a) Rechtsstaatliche Standards auf internationaler Ebene}

In Ausnahmefällen beziehen sich internationale Verwaltungsentscheidungen auf ein bestimmtes Individuum. Regelmäßig sind sie dann schon so konkret ausgestaltet, dass rechtsstaatliche Anforderungen wie Anhörung oder Rechtsschutz im Zusammenhang mit der Umsetzung bzw. dem Vollzug auf nationaler Ebene angemessen nicht mehr gewährleistet werden können, ohne dass die einschlägige völkerrechtliche Verpflichtung selbst in Frage gestellt wird. Zunächst gehört die Verwirklichung der Menschenrechte zu den grundlegenden Zielen der UN-Charta (Art. 1 Ziff. 3), die auch für die Arbeit der UN-Organe verbindlich sind. ${ }^{97}$ Wenn aber internationales Recht, vor allem die Menschenrechts-

\footnotetext{
${ }_{94}$ Hier wird traditionell mit der Figur der ,antezipierten Sachverständigengutachten “ argumentiert (BVerwGE 72, 300 [320]; 107, 338 [340ff.]). Die damit verbundene Argumentation greift jedoch zu kurz, da Experten eben keine Abwägung vorzunehmen haben. $\mathrm{Zu}$ verschiedenen Ansätzen im Einzelnen etwa E. Pache Tatbestandliche Abwägung und Beurteilungsspielraum, 2001, insbesondere 479ff.; U. Di Fabio Risikoentscheidung im Rechtsstaat 1994, insbesondere 460; vor allem auf funktionalrechtliche Gesichtspunkte abstellend C.D. Classen Die Europäisierung der Verwaltungsgerichtsbarkeit, 1996, $134 \mathrm{ff}$.

${ }_{95}$ Dazu bereits in Fn. 77.

${ }_{96}$ Dazu bereits in Fn. 66.

${ }^{97}$ Siehe zum Sicherheitsrat Art. 24 Abs. 2 UN-Charta; zu dessen Menschenrechtsbindung E. de Wet/A. Noellkaemper GYIL 45 (2000), 166 (181); A. Reinisch AJIL 95 (2001), 851 (853 ff.); Stahn ZaöRV 51 (2001), 107. (139); T. Schilling ZaöRV 64 (2004), 343 (345ff.); Payandeh ZaöRV 66 (2006) 41 (48); C. Ohler EuR 2006, 848 (857 f); C. Tomuschat EuGRZ 2007, 1 (6). Mit Blick auf den Kosovo sieht die Resolution des Sicherheitsrats 1244 ausdrücklich eine Bindung an die Menschenrechte vor; dazu Stahn ebd., $150 \mathrm{ff}$. Außerdem wurde durch die UN-Verwaltung auch eine Gerichtsbarkeit errichtet; dazu Garcia RGDIP 104 (2000), 61 (68f.); zum Rechtsschutz dort Stahn ebd. $161 \mathrm{ff}$. Zur begrenzten Möglichkeit gerichtlicher Kontrolle siehe noch in Fn. 100 und 101. Ergän-
} 
pakte, von den nationalen Stellen die Einhaltung gewisser Standards, insbesondere die Gewährleistung von Rechtsschutz, verlangt und zudem nationale Stellen - auch Gerichte - nicht durch einseitige Entscheidungen internationale Verpflichtungen in Frage stellen dürfen, kann die Konsequenz nur in der Bindung auch der internationalen Organe an die Menschenrechte liegen. Hierzu gehört im Grundsatz auch die Pflicht zur Rechtsschutzgewähr.98 So hat denn der EGMR im Zusammenhang mit der Frage, ob internationale Verpflichtungen eine Einschränkung der Rechtsschutzgarantie des Art. 6 EMRK rechtfertigen können, als Bedingung hierfür regelmäßig äquivalente anderweitige Schutzmaßnahmen verlangt. ${ }^{99}$ Gewisse, im Rechtsvergleich anerkannte Ausnahmen bei der Pflicht zur Rechtsschutzgewähr etwa im Sicherheitsbereich ${ }^{100}$ oder auch funktionale Grenzen der gerichtlichen Kontrolle ${ }^{101}$ sind aber ggf. auch im vorliegenden Kontext zu berücksichtigen.

zend ist darauf hinzuweisen, dass Berichte an den Menschenrechtsausschuss verfasst wurden, die für diesen Anlass zu Empfehlungen für die Zukunft waren. Im Zusammenhang mit Rechtsschutz für UN-Beamte hat der IGH sogar ausdrücklich auf die ChartaZiele Bezug genommen; siehe ICJ-Reports 1954, 47 (57).

98 Siehe Art. 14 IPbürgR. Zum elementaren Charakter gerade der Rechtsschutzgarantie auch im vorliegenden Kontext Stahn ZaöRV 51 (2001), 107 (137ff.).

99 EGMR, Slg. 1999-I, $393 \$ 67$ ff. - Waite und Kennedy; vgl. auch allgemein EGMR, Slg. 2005-VI - Bosphorus, $\$ \$ 152 \mathrm{ff}$.

100 Siehe zur Sicherheit nur Art. 10 Abs. 2 S. 2, Art. 19 Abs. 4 S. 3 GG sowie (zu Art. 13 EMRK) EGMR, Slg. A $28 \$ 68 \mathrm{ff}$. - Klass; allgemein auch dazu Kingsbury/ Krisch/Stewart Law and Contemporary Problems 68 (2005), 15 (42); ferner zu den

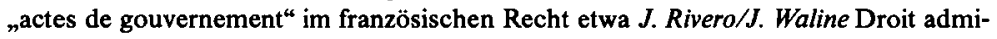
nistratif, 20 Aufl. 2004, Rn. $338 \mathrm{ff}$; G.Lebreton Droit administratif général, 3. Aufl. 2004, Rn. $62 \mathrm{ff}$. Mit Blick auf eine gerichtliche Kontrolle des Sicherheitsrates ist auch die besondere Bedeutung der Aufgabe der Friedenswahrung zu beachten, als deren Teil sich der Menschenrechtsschutz darstellt. Ganz allgemein zu Grenzen der Rechtsschutzgewährleistungen siehe (zu Art. 6 EMRK, konkret im Zusammenhang mit Immunitätsregelungen) EGMR, 2001-XI, 37 - Mc Elhinney, \$§ 39 f. (mit explizitem Hinweis, dass Rechtsschutz im beklagten Staat möglich und zumutbar sei); 79 - Al-Adsani, $\$ \S 53 \mathrm{ff}$.; 157 - Fogarty, $\$ 37$ f.; 2002-X, 389 - Kalgeropoulou, $\$ \S 401$ f.; ferner in vorheriger Fn.

${ }^{101}$ Dies gilt insbesondere mit Blick auf eine gerichtliche Kontrolle des Sicherheitsrates; zurückhaltend zu dieser Nolte (Fn 1), Leitsatz 6 und II 1. Im Rahmen der gerichtlichen Kontrolle von (gemeinschaftlichen) Umsetzungsakten von Resolutionen des UN-Sicherheitsrates nimmt das EuG eine Kontrolle am Maßstab des ius cogens vor, siehe Slg. 2005, II-3533, Rs. T-306/01 - Yusuf, Rn. 277 ff.; für eine Kontrolle am Maßstab der normalen EG-Grundrechte hingegen GA Maduro, R. C-402/05 P, Rn. $17 \mathrm{ff}$. Das entsprechende Urteil des EuGH stand zum Zeitpunkt des Abschlusses dieses Beitrages noch aus. Dem EuG zustimmend Tomuschat EuGRZ 2007, 1 (10). Überzeugend plädiert Payandeh ebd., 58, eher für eine Evidenzkontrolle; zurückhaltend überhaupt 
Bei Beantwortung der Frage, wie stark ein fremdes mit dem eigenen Schutzsystem vergleichbar sein muss, sind die Argumente auch aus der nationalen Diskussion bekannt. Einerseits soll die verfassungsrechtliche Identität gewahrt werden; andererseits sind auch fremde Rechtsordnungen zu respektieren; ${ }^{102}$ das Beharren auf eigenen Standards macht nämlich jegliche internationale Kooperation unmöglich. Die zuletzt genannten Argumente rechtfertigen jedoch nicht ein Abweichen von universell geltenden Menschenrechten.

Konkret sind rechtsstaatliche Standards wie bei der staatenbezogenen Verwaltung zunächst im positiven Recht zu suchen. So sehen etwa das Seerechtsübereinkommen ${ }^{103}$ oder die Rheinschifffahrtsakte ${ }^{104}$ entsprechende Rechtsschutzmöglichkeiten vor. Die einschlägige Rechtsordnung aber ist häufig lückenhafter als das nationale Recht. Die Konsequenz von Fehlern in einem Verwaltungsverfahren ${ }^{105}$ etwa dürfte selten ausdrücklich geregelt sein. Dementsprechend sind auch hier allgemeine Rechtsgrundsätze im Sinne von Art. 38 Abs. 1 lit. c IGH-Statut herauszubilden. ${ }^{106}$ Internationale Gerichte, die individualbezogene Verfahren zu entscheiden haben, haben dies denn auch regelmäßig getan. Zum in-

gegenüber einer Kontrolle an völkerrechtlichen Maßstäben $M$. Nettesheim CMLRev. 44 (2007), 567 (587). Demgegenüber hat der EGMR in einer jüngst ergangenen Entscheidung vom 2. 5. 2007 (verbundene Beschwerden 71412/01 - Behrami - und 78166/01 - Saramati) eine Überprüfung von Entscheidungen von KFOR und UNMIK am Maßstab der EMRK trotz Bindung der beteiligten Staaten abgelehnt, da das Handeln von KFOR und UNMIK der UNO zuzurechnen sei und zudem hier nicht nationale Behörden im eigenen Hoheitsgebiet gehandelt hätten (Rn. $147 \mathrm{ff}$.); dem zustimmend Nolte (Fn. 1), ebd. Zumindest für die KFOR ist die Argumentation zweifelhaft, da deren Angehörige sogar nach Ziff. 2 der einschlägigen regulation 2000/47 der Gerichtsbarkeit ihrer jeweiligen Herkunftsstaaten unterstehen (Stahn, ZaöRV 51 [2001], 107 [163]). Auch wird weder nach äquivalentem Schutz (dazu Fn. 99) durch KFOR und UNMIK als Voraussetzung für eine Beteiligung an diesen gefragt noch geprüft, ob internationale Standards beachtet wurden: was mit diesen nicht zu vereinbaren ist, kann kaum „in einer demokratischen Gesellschaft notwendig“ sein im Sinne der Schranken der EMRK-Garantien.

102 BVerfGE 37, 271 (277ff.); 73, 339 (366ff.); 102, 147 (164ff.); ferner zum internationalen Rechtshilfeverkehr BVerfGE 75, 1 (16f.); 108, 129 (137); 113, 154 (162f.).

${ }^{103}$ Art. 186 SRÜ; XI Abschnitt 5; XI und Annex VI Abschnitt VI; dazu Wolfrum (Fn. 21), $607 \mathrm{fr}$.

${ }^{104}$ Art. 41 lit. c iVm Art. 37.

105 Deren unterschiedliche Gewichtung (dazu sogleich im Text) haben Konsequenzen für deren Entscheidungserheblichkeit; dazu (im EG-Kontext) etwa C.D. Classen Die Verwaltung 31 (1998), 307 (322ff.).

106 Allgemein dazu etwa Kingsbury/Krisch/Stewart Law and Contemporary Problems 468 (2005), 15 (40ff.). 
ternationalen Beamtenrecht ${ }^{107}$ wurden von den internationalen Dienstgerichten ${ }^{108}$ Grundsätze entwickelt wie der Schutz vor Diskriminierung ${ }^{109}$ und, in Form des Schutzes von wohlerworbenen Rechten, ein Äquivalent zum Vertrauensschutz ${ }^{110}$, aber auch Verfahrensstandards ${ }^{111}$. Ähnlich verfahren die Strafgerichtshöfe der UNO für Jugoslawien und Ruanda. ${ }^{112}$

Wegen divergierender Rechts- und Wertvorstellungen ist dies sicherlich kein leichtes Unterfangen. ${ }^{113}$ Allerdings musste und muss schon der EuGH bei der Entwicklung des europäischen Verwaltungsrechts mit beträchtlichen Unterschieden im Rechtsverständnis fertig werden:114 Dem kontinentaleuropäischen Recht liegt vielfach ein kodifikatorischer Gedanke zugrunde; es ist formal von einer besonderen demokratischen Le-

${ }^{107}$ Zur Bedeutung der allgemeinen Rechtsgrundsätze für das internationale Dienstrecht siehe $J$. Kolasa in: R.J. Dupuy (Hrsg.) Manuel sur les organisations internationales, 1988, 210 (225); Schermers/Blokker (Fn. 19), § 539; A. Plantey Droit pratique de la fonction publique internationale, 1977, Rn. $181 \mathrm{ff}$; $M . B$. Akehurst The Law Governing Employment in International Organizations, 1967, 72ff.; H.J. Priess Internationale Verwaltungsgerichte und Beschwerdeausschüsse, 1989, $110 \mathrm{ff}$.; C. Apprill RGDIP 87 (1983), $315 \mathrm{ff}$.

${ }^{108} \mathrm{Zu}$ nennen sind vor allem die Verwaltungsgerichte der UN und der ILO, die jeweils auch für andere UN-Sonderorganisationen tätig sind; dazu und zu weiteren einschlägigen Institutionen Seidl-Hohenveldern/Loibl (Fn. 19), Rn. 1351 ff.; Amerasinghe ICLQ 45 [1996], 773 (778ff.); ausführlich $K$. Wellms Remedies against International Organisations; 1996; Priess (Fn. 107), 11 ff.; A. Pellet RGDIP 85 (1981) 253 ff.; I. SeidlHohenveldern FS Schlochauer, 1981, $615 \mathrm{ff}$.

${ }^{109}$ C.F. Amerasinghe ZaöRV 44 (1984), 439 (442ff., $454 \mathrm{ff}$ ).

${ }^{110}$ Diese begrenzen die Möglichkeit der Internationalen Organisationen, einseitig die jeweiligen Beamtenstatute zu ändern. Siehe dazu etwa Akehurst (Fn. 107), 208 ff.; Priess (Fn. 107), $110 \mathrm{ff}$.

${ }_{111}$ Amerasinghe ICLQ 45 [1996], 773 (788); ders. ZaöRV 44 (1984), 439 (462ff.).

112 Dazu W. Weiss AVR 39 (2001), 394 (420ff.).

${ }^{113}$ Dazu Kingsbury/Krisch/Stewart Law and Contemporary Problems 68 (2005), 15 (29ff., 46f.). Skeptisch daher Harlow EJIL 17 (2006), 187 (207f.). Vgl. auch Schorkopf (Fn. 84), § $14 \mathrm{I}$.

114 Klassisch A.V. Dicey Introduction to the study of the Constitution, 10. Aufl. 1959, Kap. XII (deutsch: in: G. Robbers [Hrsg.] Dicey, Einführung in das Studium des Verfassungsrechts, 2002); Schmidt-ABmann Der Staat 45 (2006), 315 (335). - Ähnlich wie auf internationaler Ebene hat das Beamtenrecht auch in der EG maßgebliche Impulse für die Entwicklung des Verwaltungsrechts insgesamt geliefert; dazu etwa $A$. Hatje Der Rechtsschutz der Stellenbewerber im europäischen Beamtenrecht, 1988. Während in der EG allgemeine Rechtsgrundsätze auch herausgearbeitet werden können, die sich formal nur auf wenige Rechtsordnungen stützen (Weiss AVR 39 [2001] 394 [410]; Beispiel: EuGH, Slg. 1982, 1575, Rs. 155/79 - AM \& S, Rn. 18ff.), setzten internationale Standards eine internationale Anerkennung voraus (Weiss ebd., 405). 
gitimation zumindest grundsätzlicher Rechtssetzung geprägt ${ }^{115}$ und inhaltlich von einem systematischen Ansatz. ${ }^{116}$ Demgegenüber steht in der angloamerikanischen Welt des Common Law die Bewährung des Rechts in der Praxis im Vordergrund. ${ }^{17}$ Das französische Verwaltungsrecht geht von einer prinzipiellen Sonderstellung der öffentlichen Verwaltung im Rechtssystem aus, was im Grundsatz jede Heranziehung bürgerlichrechtlicher Vorschriften ausschließt. ${ }^{118}$ Nach dem Common Law hingegen soll die Verwaltung in einem freiheitlich-rechtsstaatlichen System über möglichst wenige Sonderbefugnisse verfügen.119 Auch wenn es dem deutschen Recht gelungen ist, bestimmte, schon erwähnte materiellrechtliche topoi auf europäischer und internationaler Ebene zu platzieren, muss es sich aber doch auf eine insgesamt stärker verfahrensrechtliche Struktur einstellen, als sie im deutschen Recht üblich ist. ${ }^{120}$

115 Dazu A. v. Bogdandy Gubernative Rechtssetzung, 2000, passim.

116 Dazu E. Schmidt-Aßmann Das allgemeine Verwaltungsrecht als Ordnungsidee, 2. Aufl. 2004, § $1 \mathrm{Rn} .3 \mathrm{ff}$.

117 Instruktiv dazu (mit Blick auf die USA) O. Lepsius, in: H. Schulze-Fielitz (Hrsg.) Staatsrechtslehre als Wissenschaft, 2007, 319, 320ff., ferner D. Melin Gesetzesauslegung in den USA und in Deutschland, 2005; zu USA und Großbritannien R. David/C. Jauffret-Spinosi Les grands systèmes du droit contemporains, 11. Aufl. 2002, Rn. 268ff., 326; U. Zweigert/H. Kötz Einführung in die Rechtsvergleichung, 3. Aufl. $1996250 \mathrm{ff}$.; mit Blick auf England, das allerdings durch die europäische Integration besonderen $\mathrm{Be}-$ dingungen unterliegt, nuancierend $S$. Vogenauer Die Auslegung von Gesetzen in England und auf dem Kontinent, 2001, $1295 \mathrm{ff}$.

118 Das zeigt vor allem das Regime verwaltungsrechlicher Verträge mit Privaten, vor allem über die Erbringung von Dienstleistungen. $\mathrm{Zu}$ den grundsätzlichen Unterschieden zum Zivilrecht, die insbesondere in einem auch unabhängig von konkreten Regelungen bestehenden Recht der Verwaltung zur einseitigen Änderungen vertraglicher Verpflichtungen eines Auftragnehmers bestehen - dieser hat nur ein Recht auf Wahrung des finanziellen Gleichgewichts - siehe Rivero/Waline(Fn. 100), Rn. 439 ff.; Lebreton (Fn. 100), Rn. $187 \mathrm{ff}$. In diesem Kontext ist auch zu sehen die ,conception française de la séparation des pouvoirs“; dazu Conseil constitutionnel, 86-224 DC cons. 15, mit Anmerkung abgedruckt in $L$. Favorew $L$. Philip Les grandes décisions du Conseil constitutionnel, 11. Aufl. 2001, Nr. 41, sowie 89-261 DC cons. 19, nach der die Verwaltung nur in Ausnahmefällen der Kontrolle durch die ordentliche Gerichtsbarkeit unterworfen werden darf.

119 Zum englischen Recht W. Wade/C. Forsyth Administrative Law, 8. Aufl. 2000, 24, $34 \mathrm{ff}$;; vgl. auch (zu den USA) $O$. Lepsius Verwaltungsrecht unter dem Common Law, 1997 , insbesondere $37 \mathrm{ff}$.

$120 \mathrm{Vgl}$. schon oben in Fn. 105. Während etwa in Deutschland diskutiert wird, inwieweit aus materiellen Grundrechten Verfahrensgarantien abgeleitet werden können (klassisch dazu $P$. Häberle Grundrechte im Leistungsstaat, VVDStRL 30, $43 \mathrm{ff}$.), wurden im US-amerikanischen Recht aus der due-process-clause des XIV. Amendments zur USVerfassung materielle Garantien abgeleitet (,substantive due process“); dazu G. Gunther Constitutional Law 12. Aufl. 1991, 432 ff.; J.A. Barron/C.T. Dieners Constitutional Law, 1991, 143 ff.; W. Brugger Einführung in das öffentliche Recht der USA, 2. Aufl. 2001, § 11; 
Wenn der EuGH trotzdem in seinem Aufgabenbereich ein überzeugendes Modell entwickeln konnte, so deshalb, weil er sich weniger mit methodischen Herleitungen als mit konkreten Ergebnissen befasst hat. ${ }^{121}$ Internationale Spruchkörper können ebenso verfahren. 122

\section{b) Rückgriff auf nationale Strukturen}

Teilweise kann auch auf nationale Mechanismen zurückgegriffen werden. Begründet ein Rechtsfehler auf internationaler Ebene im Verhältnis zu einem für ein Individuum verantwortlichen Staat einen Ersatzanspruch, kann dieses ggf. nach nationalem Recht Erstattung verlangen. ${ }^{123}$ Nationales Recht hilft auch, wenn auf internationaler Ebene keine eigenständige Entscheidung getroffen wird. Bei Interpol etwa liegt die Verantwortung für die Richtigkeit der Daten bei den liefernden Staaten; grundsätzlich ist also dort etwa nach Rechtsschutz (und Haftung) zu suchen. ${ }^{124}$ Fehlt es an internationalen Institutionen für die Rechtsschutzgewähr, dürfte diese auch sonst auf nationaler Ebene zu suchen sein, lassen sich doch die entsprechenden Institutionen nicht so einfach wie inhaltliche Standards durch Rechtsvergleichung generieren.125 Auch die menschenrechtlichen Verpflichtungen nehmen wie erwähnt die nationale Ebene in die Pflicht. Nahe liegt in solchen Fällen eine menschenrechtskonforme Auslegung der völkerrechtlichen Verpflichtung. Wenn also die Regelungen über den Hohen Verwalter der UN in Bosnien keine gerichtliche Kontrolle über diesen vorsehen, ist deren Ausübung durch das nationale Verfassungsgericht auch völkerrechtlich nicht zu beanstanden. ${ }^{126}$

zur Diskussion $M$. Schefer Konkretisierung von Grundrechten durch den U.S.-Supreme Court, 1991, $133 \mathrm{ff} ., 139 \mathrm{ff}$.

121 Dies gilt ganz allgemein für eine erfolgreiche Rechtsvergleichung; siehe etwa U. Kischel ZVglRWiss. 104 (2005), $10 \mathrm{ff}$.

122 Friedmann (Fn. 8), 161; vgl. auch schon in Fn. 107 ff., aber auch Fn. 45.

${ }^{123}$ Im Fall des internationalen Beamtenrechts besteht wegen der Unabhängigkeit des Beamten kein Recht auf diplomatischen Schutz, der Voraussetzung ist für die dargestellte Konstruktion; hier kommt nur ein Direktanspruch gegen die Organisation in Betracht.

124 J. Martínez Soria VA 89 (1998), 400 (404f.); zum (fehlenden) Rechtsschutz gegen Interpol vor deutschen Gerichten ebd., 406; Randelzhofer FS Schlochauer, 531 (554). Dagegen kann gegen eine Verwendung oder Weitergabe durch deutsche Behörden von Daten, die von Interpol (zunächst an das BKA; vgl. § 3 Abs. 2 BKAG) übermittelt wurden, vor deutschen Verwaltungsgerichten Klage erhoben werden. Kritisch zum fehlenden Rechtsschutz gegen Interpol selbst C. Eick/A. Trittel EuGRZ 1985, 81 (83).

${ }^{125}$ M. Ruffert in: Möllers/Voßkuhle/Walter (Fn. 5), 395 (409).

126 Siehe das entsprechende Urteil des Verfassungsgerichts von Bosnien-Herzegowina, ZaöRV 51 (2001), 173 ff. Dessen zentrales Argument bestand allerdings in dem 


\section{c) Insbesondere: der Austausch personenbezogener Daten}

Für den internationalen Austausch von personenbezogenen Daten im speziellen ist nach deutschem Recht eine gesetzliche Grundlage erforderlich. ${ }^{127}$ Nach den verschiedenen Regelungen (insbesondere $\S 4 \mathrm{~b}$ BDSG) ${ }^{128}$ hat eine Datenweitergabe im Regelfall zu unterbleiben, wenn ein angemessenes Schutzniveau, insbesondere eine grundsätzliche Zweckbindung ${ }^{129}$, nicht besteht. Durch näher definierte Gegengründe kann diese Hürde aber im Rahmen der Verhältnismäßigkeit überwunden werden. Grundsätzlich ist der Betroffene über eine Datenweitergabe $\mathrm{zu}$ informieren ( $\$ 4 \mathrm{~b}$ Abs. 4) ${ }^{130}$ und kann dann auch klagen; eine Haftung der deutschen Stelle für einen späteren Datenmissbrauch kommt in Betracht, wenn diese nicht alles Zumutbare zu dessen Verhinderung ge$\tan$ hat ( $\$ 7$ BDSG).

Umgekehrt dürfen Daten von ausländischen Stellen nur entgegen genommen und verarbeitet werden, wenn sie unter rechtsstaatlich akzeptabeln Voraussetzungen gewonnen wurden; durch Folter erpresste Aussagen sind daher unverwertbar. ${ }^{131} \mathrm{Zu}$ beachten ist aber auch, dass anders

Hinweis, dass der UN-Verwalter funktional nationale Staatsgewalt ausübe und deswegen das Verfassungsgericht zur Kontrolle berufen sei; ähnlich die Beschreibung der Stellung der UN-Behörden bei $M$. Ruffert ICLQ 50 (2001), 613 (626). Zur konkreten Entscheidung Stahn ZaöRV 51 (2001), 107 (166ff.); Kingsbury/Krisch/Stewart Law and Contemporary Problems 68 (2005), 15 (32); ausführlich Rehs (Fn. 22).

127 M. Baldus Transnationales Polizeirecht, 2001, $191 \mathrm{ff}$;; allgemein dazu auch BVerfGE 65, 1 (44).

128 Daneben stehen bereichsspezifische Bestimmungen; siehe etwa $\$ 14$ BKAG (und dazu Baldus [Fn. 127] 292 ff.), 50b GWB, 77 SGB X sowie die auf Art. 26 des OECDMusterabkommens beruhenden Regelungen in den Doppelbesteuerungsabkommen; Übersicht bei $M$. Engelschalk in: Vogel/Lehner (Fn. 10), Art. 26 Rn. 73.

129 Dazu etwa $\S \S 4$ b Abs. $1 \mathrm{iVm} 15$ Abs. 3 BDSG, und im Einzelnen etwa S. Simitis in: ders. (Hrsg.) BDSG, $\S 4 \mathrm{~b}$ Rn. 52 ff.; groBzügiger P. Gola/R. Schomerus BDSG, 9. Aufl. 2007, § 4b Rn. 12; H.P. Bull in: M. Baldus/M. Soiné (Rechtsprobleme der internationalen polizeilichen Zusammenarbeit, 1999, 9 [15ff.]). Die Verpflichtung zur Information des Empfängers über die Zweckbindung ( $\$ 4 \mathrm{~b}$ Abs. 6 BDSG) entbindet nicht von der materiellen Bindung an diese; Simitis, ebd., Rn. 58; tendenziell großzügiger Ohler (Fn. 12), 306. Zur Datenübermittlung an Interpol kritisch wegen unzureichender Datenschutzstandards Baldus (Fn. 127), 319f. Als spezialgesetzliche Regelungen siehe etwa $\$$ 50b Abs. 2 GWB und 78 SGB X sowie im zwischenstaatlichen Kontext zum Doppelbesteuerungsrecht Art. 26 des OECD-Musterabkommens und dazu Engelschalk (Fn. 128), Art. 26 Rn. 82. Zum verfassungsrechtlichen Rahmen Baldus ebd., $206 \mathrm{ff}$; $T$ Tomuschat (Fn. 73), Rn. 62.

130 Dazu Simitis (Fn. 129), $\$ 4 b$ Rn. 85 ff.

131 Schmidt-ABmann Der Staat 45 (2006), 315 (334); im Ergebnis ähnlich (Art. 25 GG als Maßstab) Baldus (Fn. 127), 229ff., 326ff. Die Verwertung ausländischer Vernehmungsprotokolle in Strafverfahren ist grundsätzlich möglich, wenn das ausländische 
als im innerstaatlichen Recht zu hohe Standards nicht durchsetzbar sind, sondern dazu führen, dass Informationen gar nicht verwertet werden können bzw. dürfen. ${ }^{132}$

\section{Horizontale Internationalisierung der Verwaltung}

\section{Anknüpfungen und Verweisungen \\ a) Anknüpfung an ausländische Sachverhalte}

Bei der Herausbildung des heutigen Verwaltungsrechts in der zweiten Hälfte des 19. Jahrhunderts stand der Gebietsbezug klar im Vordergrund. ${ }^{133}$ Heute hingegen ist der Blick über die Grenze selbstverständlich geworden: Ausländische Einkünfte sind bei der Entscheidung über die Gewährung von Sozialleistungen im Inland zu berücksichtigen, ${ }^{134}$ ausländisches Verhalten bei der Entscheidung über im Inland wirkende Wettbewerbsbeschränkungen ( $\$ 130$ Abs. 2 GWB). Bei der Feststellung einer Asylberechtigung nach Art. 16a GG hat sich zumeist sogar der gesamte Sachverhalt im Ausland abgespielt.

Deutsche Behörden können aber auch zum Schutz ausländischer und internationaler Rechtsgüter tätig werden. Verfassungsrechtlich verpflichtend wirkt Art. 26 Abs. 1 Satz 1 GG, wonach jede Störung des friedlichen

Recht beachtet und wesentliche Grundsätze rechtsstaatlichen Verfahrens eingehalten wurden (BGH StV 1982, 153 [154]; NStZ 1983, 181). Weiterhin muss sich der deutsche Richter darum bemühen, dass das ausländische Gericht soweit möglich auch deutschen Standards Rechnung trägt (BGH, StV 1988, 563); ist eine Nachholung einer Verfahrenshandlung in Deutschland möglich (konkret: die Belehrung der Verlobten eines Angeklagten über das ihr nur nach deutschem Recht zustehende Zeugnisverweigerungsrecht), muss dies geschehen (BGH, NStZ 1993, 394). Enger, für eine grundsätzliche Bindung an die inländischen Vorgaben (nur Mittel und Ziele, die auch in Deutschland rechtmäßig sind) Simitis (Fn. 129), Rn. 99. Zur Unverwertbarkeit von Folteraussagen auch House of Lords, EuGRZ 2006, 63.

132 Sind die Maßstäbe für die Erhebung von Informationen großzügiger als in Deutschland, sind ggf. aber auch besondere ausländische Schutzvorschriften etwa zu den Rechtsfolgen unzulässig gewonnener Beweismittel mit zu beachten, die deutsche Recht nicht kennt. Ein Beispiel bildet das besonders strenge Beweisverwertungsverbot des US-amerikanischen Strafprozessrechts (dazu C. Roxin Strafverfahrensrecht, 25. Aufl. 1998, § $25 \mathrm{Rn} .42$ ).

${ }_{133}$ Siehe etwa O. Mayer(Fn. 8), 453. Im Weiteren werden allerdings auch Ausnahmen anerkannt.

$134 \S 2$ der VO zu $\S 82$ SGB XII verweist auf den Einkunftsbegriff des EStG; dieser wiederum erfasst das Welteinkommen; siehe S.F. Seeger in L. Schmidt, EStG, 22. Aufl. 2003, § 2 Rn. 4. 
Zusammenlebens der Völker verfassungswidrig ist. Art. 1 Abs. 2 GG macht deutlich: hier geht es nicht nur um die Verhinderung eines Krieges; auch sonstige elementare Regeln menschlichen Zusammenlebens werden erfasst. ${ }^{135}$ Die verwaltungsrechtliche Bedeutung dieser Bestimmung ist bislang noch nicht ausgeleuchtet. Völkerrechtliche Verpflichtungen enthalten die nach Art. 25 GG auch innerstaatlich beachtlichen gewohnheitsrechtlichen Regeln über die Ordnungsaufgabe des Staates ${ }^{136}$ sowie das Nachbarrecht ${ }^{137}$, ferner zahlreiche Verträge ${ }^{138}$. Ein ausdrücklicher gesetzlicher Hinweis auf den Einschluss ausländischer und internationaler Rechtsgüter ist in all diesen Fällen nicht erforderlich. ${ }^{139} \mathrm{Zu}$ Recht hat daher das BVerwG die Klage eines in den Niederlanden ansässigen Bürgers gegen die Genehmigung eines Kernkraftwerkes, das etwa $25 \mathrm{Ki}$ lometer von seinem Wohnsitz entfernt, aber in Deutschland errichtet werden sollte, als zulässig angesehen. ${ }^{140}$ Wird hingegen der Anwendungs-

135 Für einen weiten Friedensbegriff auch Stoll DVBl. 2007, 1064 (1070); Pernice (Fn. 61), Art. 26 Rn. 15; zurückhaltend U. Fink in: v. Mangoldt/Klein/Starck (Fn. 61), Art. 26 Rn. 20f; ausführlich zum Friedensbegriff des Grundgesetzes $N$. Bringmann Völkerfriede durch Strafbewehrung nach dem Grundgesetz für die Bundesrepublik Deutschland, 2005; S. Schiedermair Der internationale Frieden und das Grundgesetz, 2005.

$136 \mathrm{Zu}$ dieser etwa IGH, ICJ Reports 1949, 4 (23) - Korfu-Kanal. Zu den Konsequenzen für das Polizeirecht wie hier Ohler (Fn. 12), 310; Mokros (Fn. 31), Rn. O 47; weitergehend $K$. Vogel Der räumliche Anwendungsbereich der Verwaltungsrechtsnorm, 1965, der (unter insoweit wohl nicht tragender Berufung auf den Grundsatz der offenen Staatlichkeit) im Bereich der „öffentlichen Sicherheit und Ordnung“ grundsätzlich die gesamte Rechtsordnung ausländischer Staaten ins Auge fassen will und dies nur anders bewertet, wenn es nicht um die äußere Ordnung, sondern um die Sozialgestaltung geht (419).

${ }^{137}$ Zum völkerrechtlichen Umweltrecht etwa $R$. Wolfrum, DVB1. 1984, 493 (499f.); M. Bothe, UPR 1983, $1 \mathrm{ff}$; Durner (Fn. 64), $149 \mathrm{ff}$.

138 Siehe etwa zum Seerecht Art. 218 SRÜ und dazu BVerfGE 112, 1 (26f.).

${ }^{139}$ R. Wahl in: F. Schoch/R. Pietzner/E. Schmidt-ABmann (Hrsg.) VwGO, $\$ 42$ Abs. 2 Rn. 223: Seine entsprechende Kritik am einschlägigen Urteil des BVerwG (nachf. Fn.) findet im Urteil keine Grundlage; dort wird $\$ 1$ Nr. 4 AtG zwar mit seinem Verweis auf das Völkerrecht herangezogen, aber nur zur „Bestätigung“ des zuvor bereits gefundenen Ergebnisses. AA Dieckert (Fn. 76), $197 \mathrm{ff}$., $207 \mathrm{ff}$.; T. Oppermann/M. Kilian, Gleichstellung ausländischer Grenznachbarn in deutschen Verwaltungsverfahren, 1981. - Daneben sind ggf. völkerrechtliche Schranken zu beachten, etwa aus Verträgen wie dem WTO-Recht. So dürfen Maßnahmen zum Schutz von Tieren außerhalb der eigenen Jurisdiktion nicht ohne weiteres ohne internationale Abstimmung getroffen werden; siehe die Entscheidung des DSB im die USA betreffenden shrimps-Fall (Fn. 45).

140 BVerwGE 75, 285 (288f.). Das erstinstanzlich entscheidende VG Oldenburg (DVBl. 1985, 802) hatte unter Hinweis auf das völkerrechtliche Territorialitätsprinzip gegenteilig entschieden: deutsches Recht gelte nicht im Ausland. Zumindest in der Begründung war das verfehlt: Das Völkerrecht verbietet zwar im Grundsatz die Vornahme 
bereich der verfassungsrechtlichen und der in Deutschland anwendbaren völkerrechtlichen Regeln verlassen, muss der Gesetzgeber die Relevanz ausländischer Rechtsgüter ausdrücklich anordnen.

\section{b) Anknüpfung an und Verweisung auf ausländische Rechtslagen}

Daneben knüpft das deutsche Recht in verschiedenen Zusammenhängen an bestimmte ausländische, im Regelfall durch Einzelentscheidung fixierte Rechtslagen an. ${ }^{141}$ Klassischer Fall ist das Recht der Doppelbesteuerung - der deutsche Staat erhebt auf bestimmte, meist ausländische Einkünfte keine oder niedrigere Steuern, weil diese bereits im Ausland versteuert wurden.142 Die Anerkennung ausländischer Führerscheine ${ }^{143}$ bildet ein weiteres Beispiel. ${ }^{144}$ Voraussetzung sind eine ge-

von Hoheitsakten auf fremdem Staatsgebiet, die ,jurisdiction to enforce". Demgegenüber darf bei der Vornahme von Hoheitsakten auf eigenem Staatsgebiet grundsätzlich auch an Tatbestände angeknüpft werden, die außerhalb des eigenen Hoheitsgebietes verwirklicht werden - njurisdiction to prescribe“. Zum konkreten Fall Wahl (Fn. 139), Rn. 222, allgemein dazu etwa $\operatorname{Ohler}$ (Fn. 12), 327 ff. Zu (nur über das Völkerrecht lösbaren) Folgeproblemen, etwa der Möglichkeit einer Präklusion ausländischer Bürger auch, wenn an deren Wohnsitz keine öffentliche Bekanntmachung des entsprechenden Vorhabens erfolgte, siehe Durner (Fn. 64), 158.

${ }_{141} \mathrm{Zu}$ Einzelheiten (am Bespiel des Doppelbesteuerungsrechts) ausführlich Reimer (Fn. 10), $199 \mathrm{ff}$.

${ }^{142}$ Als Methoden stehen die Freistellung ausländischer Einkünfte und die Anrechnung ausländischer Steuern zur Verfügung; siehe dazu einerseits die Doppelbesteuerungsabkommen mit anderen Staaten und dort die auf Art. $23 \mathrm{~A}$ oder Art. B des OECD-Musterabkommens zur Doppelbesteuerung beruhenden Bestimmungen, andererseits § 34c EStG. „Negative“ Konflikte - kein Staat will besteuern - sind demgegenüber grundsätzlich unproblematisch, weil es jedem Staat grundsätzlich frei steht, die Reichweite seiner Besteuerung zu bestimmen; dazu $K$. Vogel in: ders./Lehner (Fn. 10), Art. 1 Rn. 11 ff.; tendenziell aA Reimer (Fn. 10), 183. Bei Konflikten im Einzelfall - wegen unterschiedlicher Auslegung eines Doppelbesteuerungsabkommens will kein Staat die an sich vorgesehene Besteuerung vornehmen - geht man ggf. vom an sich vorgesehenen Freistellungs- (nur dort stellt sich das Problem) zum Anrechnungsverfahren über; siehe Art. 23 A Abs. 4 OECD-Musterabkommen. Siehe dazu S. Grotherr Grundlagen, Rn. 50, sowie Art. 23 A/23 B Rn. 162, in: H. Becker/H.D. Höppner/S, Grotherr/H.K. Kroppen (Hrsg.) DBA-Kommentar, Loseblattsammlung.

${ }^{143} \S 4$ der Verordnung vom 13. 11. 1934 über den internationalen Kraftfahrzeugverkehr.

144 Wird an einen ausländischen Verwaltungsakt angeknüpft, spricht man zum Teil von "transnationalem Verwaltungsakt" (ausführlich dazu M. Ruffert Die Verwaltung 34 [2001], $453 \mathrm{ff}$.). Soweit sich die grenzüberschreitende Wirkung nicht aus supranationalem, insbesondere Gemeinschaftsrecht ergibt, kann sie sich nur aus dem Recht des Staates ergeben, in dem die Wirkung erzielt werden soll (Ohler [Fn. 12], 50). In solchen Fällen ist der Begriff zumindest missverständlich. - Siehe etwa auch BGHZ 95, 256 zur Konkurseröffnung im Ausland. 
setzliche Regelung und sachliche Gründe; Art. 3 GG und verfassungsrechtliche Schutzpflichten bilden die Grenze. Soweit auf deutscher Seite nicht wie bei den Führerscheinen der Normgeber gehandelt hat, sondern wie im Steuerrecht durch Verwaltungsakt entschieden wurde, ist Rechtsschutz als solches problemlos möglich. Eine inzidente Überprüfung der ausländischen Rechtslage aber kommt im Regelfall nicht in Betracht. Schon bei rein nationalen Fällen schiede diese - Stichwort: Tatbestandswirkung - grundsätzlich aus. Bei Fällen mit Auslandsbezug kommt hinzu, dass inländischer Rechtsschutz die ausländische Rechtslage nicht zu verändern vermag; das regelmäßig durch eine solche $\mathrm{Re}-$ gelung verfolgte Ziel paralleler Behandlung ${ }^{145}$ würde verfehlt. ${ }^{146}$

Ein kollisionsrechtlicher Verweis auf ausländisches Recht mit dem Ziel dessen unmittelbarer Anwendung durch deutsche Behörden ${ }^{147}$ ist dagegen grundsätzlich unzulässig. ${ }^{148}$ Die staatliche Verwaltung darf re-

${ }_{145} \mathrm{Zu}$ dieser „Entscheidungsharmonie“ etwa $K$. Vogel in: ders./Lehner (Fn. 10), Einleitung, Rn $113 \mathrm{ff}$.

146 Konsequenz: Rechtsschutz ist im Staat zu suchen, dessen Behörden den Akt erlassen haben. So auch Ruffert (Fn. 125), 476; Ohler (Fn. 12), 154; Hatje (Fn. 4), 212. Ggf. möglich bleibt eine Verfassungsbeschwerde. Mit ihr erreicht werden kann immerhin eine (ggf. sinnvolle) Beschränkung der Wirkung ausländischer Entscheidungen im Inland (Ohler ebd.).

${ }^{147}$ Zur Frage, ob kollisionsrechtliche Verweise zu einer Geltung oder nur zu einer Anwendbarkeit oder Geltung einer (ausländischen) Norm führen, siehe (im erstgenannten Sinne) M. Glaser in: Möllers/Voßkuhle/Walter (Fn. 5), 73 (108ff.); B. Schinkels Normsatzstruktur des IPR, 2007, 112ff.; aA Ohler (Fn. 12), insbesondere $139 \mathrm{ff}$.; C. v. Bar/P. Mankowski Internationales Privatrecht, Band I, 2. Aufl. 2003, § 4 Rn. 68; siehe ferner zum Streit Niedobitek (Fn. 43), 349 ff. Der Streit ist sinnvollerweise nicht durch Verwendung von Begriffen, sondern durch Erörterung konkreter Problemlagen, die sich mit den jeweiligen Auffassungen verbinden (und ggf. differenziert zu beantworten sind), zu entscheiden. Auch hier liegt eine Herausforderung für die Wissenschaft.

148 v. Bar/Mankowski (Fn. 147), § 4 Rn. 53; differenzierend $R$. Franck RabelsZ 34 (1970), $56 \mathrm{ff}$; A. Grof in: R. Mellinghoff/H.H. Trute (Hrsg.) Die Leistungsfähigkeit des Rechts, 1987, 313ff.; ferner Ohler (Fn. 12), der zwar sieht, dass bei der Verweisung auf ausländisches Recht zu unterscheiden ist zwischen der Einräumung der Befugnis an ausländische Behörden, auf der Grundlage ihres Rechts im Inland zu handeln, und des Verweises auf ausländisches Recht als maBgebliches Recht für die Tätigkeit deutscher Behörden (123f.), aber nicht (hinreichend deutlich) zwischen beiden bei der Erörterung von Sinn ( $96 \mathrm{ff}$.) und Grenze (107ff.) unterscheidet. Immerhin gesteht auch Ohler (ganz im Sinne des hier für richtig Gehaltenen) zu, dass es für die zweite Konstellation kein praktisches Beispiel gibt (123). Nationales öffentlich-rechtliches Kollisionsrecht ist daher regelmäßig „einseitig“, beruft also nur deutsches Recht zur Anwendung. Siehe dazu Vogel (Fn. 136), 200ff.; Steindorff(Fn. 12), 581; C. Feldmüller, Die Rechtsstellung fremder Staaten und sonstiger juristischer Personen des ausländischen öffentlichen Rechts im deutschen Verwaltungsprozess, 1999, 91 ff.; zurückhaltend Ohler ebd., 87 ff.; abl. un- 
gelmäßig nur im Rahmen des von den Organen der Bundesrepublik, zumindest unter ihrer Mitwirkung definierten öffentlichen Interesses tätig werden; öffentliches Recht ist grundsätzlich nur auf die Anwendung durch nationale Behörden bezogen. ${ }^{149}$ So ist zwar im Privatrecht die Anwendung ausländischen materiellen Rechts selbstverständlich. Aus gu-

ter Hinweis auf eine von ihm angenommene ungeschriebene Kollisionsnorm $A$. Dutta Die Durchsetzung öffentlich-rechtlicher Forderungen ausländischer Staaten, 2006, $394 \mathrm{ff}$. Die Legitimation zur Entwicklung dieser Norm begründet dieser mit einer anderenfalls bestehenden Regelungslücke; der von ihm kritisierte Grundsatz der Nichtdurchsetzbarkeit ausländischer öffentlich-rechtlicher Forderungen (dazu $143 \mathrm{ff}$.) werde nämlich anderenfalls doch beachtet. Damit liegt aber ein Zirkelschluss vor. Für diese unterschiedliche Behandlung von Privat- und öffentlichem Recht hat Vogel ausführlich dargelegt, dass die für das Privatrecht (zumindest früher) angenommene "Gleichwertigkeit der Privatrechtsordnungen“ (und die damit legitimierte Anwendung fremden Privatrechts; dazu insbesondere F.C. Savigny System des heutigen römischen Rechts VIII, 1849, 27 f., 108, 128 und heute v. Bar/Mankowsky, ebd., § 3 Rn. 6) jedenfalls im öffentlichen Recht nicht besteht (Fn. 136, $215 \mathrm{ff}$., $235 \mathrm{ff}$ ); so auch Ohler ebd. 132; Schinkels (Fn. 147), $35 \mathrm{ff}$. So ist auch nicht zu verkennen, dass das Grundanliegen von v. Savigny (Die „Rechtsverhältnisse [haben], in Fällen der Collision der Gesetze, dieselbe Beurtheilung zu erwarten ..., ohne Unterschied, ob in diesem oder jenem Staat das Urtheil gesprochen wird.“, ebd., 27) in einem gewissen „Grundkonflikt“ mit dem modernen Erfordernis demokratischer Legitimation richterlicher Tätigkeit steht. Zur heutigen verfassungsrechtlichen Legitimation des IPR siehe etwa Tomuschat (Fn. 73), Rn. 56; Ohler ebd. 95; ausführlich (mit manchen kritischen Konsequenzen für das geltende Recht) Schinkels (Fn. 147), $205 \mathrm{ff}$. Das BVerfG hat das Problem bislang nicht einmal ins Blickfeld genommen; siehe E 31, 58 (73); 92, 26 (48, 52) Die angesprochene Problematik zeigt sich insbesondere in der Diskussion um die Bedeutung sogenannter „Eingriffsnormen “ im IPR (dazu J. Kropholler Internationales Privatrecht, 4. Aufl. 2001, 23; aus der Rechtsprechung BGHZ 31, 367 [371]; 32, 97 [101]; 39, 220 [227]; 43, 162 [165]; aber auch 34, 269 [175ff.]; 59, 82 [85ff]]). - Wenn vielfach schon die internationale Zuständigkeit deutscher Gerichte für auf ausländisches öffentliches Recht gestützte Klagen abgelehnt wird (BGH, WM 2005, 2274 [2275f.] - zu Gebührenforderungen; BSGE 54, 250 [251] - zu sozialrechtlichen Rückforderungsansprüchen; D. Ehlers in: Schoch/ Pietzner/Schmidt-ABmann [Fn. 139], vor $\$ 40$ Rn. 65; zur Diskussion auch Feldmüller, ebd., 82ff.), liegt dies nach richtiger Auffassung daran, dass keine Prozessordnung insoweit eine Zuständigkeit eröffnet $(\S 40$ VwGO bezieht sich nur auf deutsches öffentliches Recht) und mangels „Recht“ und damit mangels dieses voraussetzender Justizgewährleistungspflicht auch nicht eröffnen muss (deutlich dazu v. Bar/Mankowski ebd., § 5 R. 77: das Recht konstituiert die Gerichte, ihre Aufgabe und ihre Macht; dazu auch C.D. Classen VA 96 [2005] 464 [469 ff.]). - Völkervertragliche Regelungen kollisionsrechtlichen Inhalts, die bestimmen, welches nationale Recht, angewandt durch die jeweiligen Behörden, jeweils auf einen Fall angewendet werden soll, sind problemlos möglich. Beispiele bilden Doppelbesteuerungsabkommen oder auch Verträge zu sozialrechtlichen Fragen bei grenzüberschreitend Beschäftigten. Dazu Glaser (Fn. 147), 82 ff.

${ }^{149}$ Dazu Niedobitek (Fn. 43), 369f. 
ten Gründen aber bleibt das Verfahrensrecht national. ${ }^{150} \mathrm{Im}$ öffentlichen Recht sind nun beide untrennbar miteinander verbunden. ${ }^{151}$ Nur in gesetzlich definierten ${ }^{152}$ Sonderkonstellationen kann auf ausländisches Recht verwiesen werden. Dies ist vor allem möglich, wenn es nicht um einseitige Ausübung von Hoheitsgewalt geht. Beispiele sind grenzüberschreitende Zweckverbände ${ }^{153}$ oder - im Gegensatz zur Annahme von Herrn Poscher - die einem einzelnen eingeräumte Befugnis, bestimmte öffentlich-rechtliche Pflichten - Beispiel Buchführungspflicht von Gesellschaften - bei internationaler Tätigkeit statt nach deutschem nach

150 Zur Anwendbarkeit inländischen Verfahrensrechts auch bei Anwendung ausländischen materiellen Rechts durch deutsche Gerichte siehe BGH NJW 1985, 553; 1992 , 438; deutlich v. Bar/Mankowski (Fn. 147), \& 5 Rn. 77 („Kernbereich der Souveränität“); ferner $M$. Radtke Der Grundsatz der lex fori und die Anwendbarkeit ausländischen Verfahrensrechts, 1982.

151 So auch Ohler (Fn. 12), 126. Die Heranziehung ausländischen Verwaltungsverfahrensrechts (dazu ebd., 127f.) wirft immerhin in aller Regel geringere Probleme auf als im Prozessrecht, da letzteres wesentlich mehr (im Ausland unerfüllbare) organisatorische Anforderungen (etwa: Anforderungen an die Richterbank; dazu v. Bar/Mankowski [Fn. 147], § 4 Rn. 76, 78) stellt als ersteres.

152 Ein Beispiel ist auch im deutsch-schweizerischen Polizeivertrag verankert; dazu bei Fn. 184. Ohler (Fn. 12) führt für dieses Ergebnis das Demokratieprinzip sowie die Grundrechte an (314f.). Es resultiert aber schon daraus, dass anderenfalls an sich einschlägige gesetzliche Regelungen des deutschen Rechts nicht eingehalten, bei Ermessensentscheidungen die diese leitenden Gesichtspunkte nicht dem an sich verbindlichen (Art. 20 Abs. 3 GG) deutschen Recht entnommen werden.

${ }^{153}$ Die Bildung öffentlich-rechtlicher Zweckverbände durch kommunale Körperschaften ist etwa vorgesehen im Anholter Abkommen der Bundesrepublik sowie der Länder Nordrhein-Westfalen und Niedersachsen mit den Niederlanden vom 23. 5.1991 (BGBl. 1993 II 843) sowie im - mit diesem in vielen Punkten wortgleichen - Mainzer Abkommen der Länder Nordrhein-Westfalen und Rheinland-Pfalz mit belgischen Körperschaften vom 8. 3. 1996 (GVBl. NRW 1996, 255), ferner im Karlsruher Abkommen zwischen Deutschland, Luxemburg, Frankreich und der Schweiz vom 23. 1. 1996 (BGB1. 1997 II 1159); dazu Niedobitek (Fn. 43), 107 ff., zum letztgenannten Abkommen auch G. Halmes DÖv 1996, 933 ff. In den Abkommen von Anholt und Mainz werden ausdrücklich ausgeschlossen Maßnahmen eines solchen Zweckverbandes gegenüber Dritten (jeweils Art. 5); diese behalten alle Ansprüche gegen den für sie „zuständigen“ Vertragspartner und können auf dieser Grundlage den entsprechenden innerstaatlichen Rechtsweg beschreiten (jeweils Art. 10 Abs. 1). Daneben haftet auch der Zweckverband (Art. 10 Abs. 2). Im Übrigen wird für den Rechtsweg auf die Vorschriften des Staates verwiesen, in dem der Beklagte seinen Sitz hat; der Vertrag mit den Niederlanden sieht darüber hinaus die Möglichkeit einer Schiedsvereinbarung vor (jeweils Art. 11). Demgegenüber enthält das Karlsruher Abkommen keine entsprechenden Begrenzungen. Siehe ferner unten bei Fn. $173 \mathrm{ff}$. 
ausländischem Recht zu erfüllen - im Sinne einer nach Internationalem Privatrecht auch sonst regelmäßig zulässigen Rechtswahl. ${ }^{154}$

\section{Behördenkooperation}

\section{a) Abstimmung zwischen Behörden verschiedener Staaten}

Die gestiegene Mobilität der Menschen hat vor allem die Polizeibehörden veranlasst, ausländische Verbindungsbeamte in Entscheidungsprozesse einzubeziehen. Hiergegen bestehen auch ohne ausdrückliche Regelung ebenso wenig Bedenken wie gegen die informelle Einbeziehung von Privatpersonen in staatliche Entscheidungsprozesse auf nationaler Ebene, wenn und soweit die Verantwortung für das jeweilige Handeln voll bei den deutschen Stellen liegt.155 Bei divergierenden Ein-

\footnotetext{
154 Als Beispiel siehe § 292a HGB a.F., in Geltung von 1998 bis 2004. Danach konnte eine Gesellschaft alternativ zu deutschen Vorgaben nach (von privaten Standardsettern erarbeiteten, aber von der US-amerikanischen Börsenaufsicht geprüften) „international anerkannten Rechnungslegungsgrundsätzen" bilanzieren. Wie hier M. Henssler/C. Slota NZG 1999, 1133 (1137); B. Großfeld NZG 1999, 1143 (1144); ausführlich J. Adolphsen RabelsZ 68 (2004), 154 (165, 174ff.); aA - Bewertung als verfassungswidrige dynamische Verweisung - neben Poscher (Fn. 70), 167; P. Hommelhoff, in: Großkommentar zum HGB III/2, 4. Aufl. 2002, § 292a Rn. 26; L. Michael in: H. Bauer/P.M. Huber/ K.-P. Sommermann (Hrsg.) Demokratie in Europa, 2005, $431 \mathrm{ff}$; P. Kirchhof ZGR 2000, 681 ff.; zurückhaltend auch Herdegen (Fn. 88), 23. Angesichts der Tatsache, dass das IPR dem Einzelnen im Vertragsrecht eine weitgehende Freiheit zur Rechtswahl überlässt (Art. 27 EGBGB) und etwa bei einer EheschlieBung mit Beteiligung eines ausländischen Partners grundsätzlich auf ausländisches Eherecht verweist (Art. 13 EGBGB), erscheint die Kritik zu pauschal. - $\$ 292$ a HGB wurde mittlerweile durch $\$ 315$ a HGB abgelöst, der auf Gemeinschaftsrecht verweist, das die Standards ausdrücklich rezipiert; damit stellt sich das Legitimationsproblem nicht mehr. - Eine weitere denkbare Konstellationen wäre eine gesetzliche Regelung zur Durchsetzbarkeit ausländischer öffentlich-rechtlicher Geldforderungen vor deutschen Gerichten (zu praktischen Beispielen siehe die in Fn. 148 zitierten Urteile; zur sachlichen Berechtigung eines solchen Vorhabens Dutta [Fn. 148], 7 ff.; Franck RabelsZ 34 (1970) 56 [70ff.]). Allgemein dazu Vogel (Fn. 136), 238. Amts- und Rechtshilfe hingegen stellen keine Ausnahmefälle dar, weil hier die Behörden letztlich nicht ausländisches Recht unmittelbar anwenden, sondern dieses lediglich als Vorfrage heranziehen; anders wohl Steindorff(Fn. 12), 582.

155 OLG Karlsruhe, NJW 1992, 642 (643f.); Schmidt-ABmann Der Staat 45 (2006), 315 (318). Nach Auffassung des OLG Karlsruhe kann bei Exzessen ausländischer Beamter ggf. die Rechtswidrigkeit der Duldung durch deutsche Beamte festgestellt werden. Im Übrigen stellen diese dann praktisch Verwaltungshelfer dar. Siehe J. Hecker AöR 127 (2002), 291 (301); C. Gramm DVB1. 1999, 1237 (1242). - Ein Beispiel bilden auch die deutsch-französischen Zentren der Polizei- und Zollzusammenarbeit (Vertrag vom 9. 10. 1997, BGB1. 1998 II 2479); dazu E. Hetger in: Baldus/Soiné (Fn. 129), 145 ff. Die Zusammenarbeit in gemeinsamen Zentren (Art. $4 \mathrm{ff}$.) soll unter Berücksichtigung
} 
schätzungen treffen deutsche Behörden ggf. Entscheidungen, die weniger von ihrer eigenen Lageanalyse bestimmt sind als vielmehr einen diplomatischen Eklat vermeiden sollen. Ist polizeiliches Handeln dann trotzdem gerechtfertigt? Nun wird die öffentliche Sicherheit auch beeinträchtigt, wenn der Staat an der Durchführung einer von ihm geplanten Veranstaltung gehindert wird. ${ }^{156} \mathrm{Da}$ ausländischen Gästen eine eigene Beurteilung der Sicherheitslage nicht verwehrt werden kann, ${ }^{157}$ werden nationale Entscheidungen insoweit - in Grenzen - durch ausländische Einschätzungen mit bedingt.

Rechtlich verankert ist eine abgestimmte Entscheidungsfindung von verschiedenen nationalen Behörden etwa im Doppelbesteuerungsrecht (Art. 25 OECD-Musterabkommen). ${ }^{158}$ Teilweise wird die Legitimität eines solchen Verfahrens für die Fälle in Zweifel gezogen, in denen die deutsche Behörde von der gefundenen Lösung nicht voll überzeugt ist. ${ }^{159}$ Es handelt sich jedoch um eine parlamentarisch gebilligte, sachgerechte, verfahrensmäßige Konsequenz des Willens, Doppelbesteuerung real zu vermeiden. Allerdings muss die Auslegung des betreffenden Abkommens vertretbar sein. ${ }^{160}$ Gerichte sind schon aus grund- und menschenrechtlichen Gründen ${ }^{161}$ nicht formal an eine solche Verständigung zwischen den Behörden gebunden, wohl aber an das in den Abkommen enthaltene Ziel der Entscheidungsharmonie. ${ }^{162}$ Richtigerweise hat auch

der jeweiligen Zuständigkeiten der Beteiligten erfolgen (Art. 3). Zur Polizeizusammenarbeit im Grenzbereich Sachsen/Polen/Tschechische Republik $U$. Bormann ebd., $157 \mathrm{ff}$.

156 BVerfG (K), NJW 2007, 2167 (2169); ferner BVerfG (K), NJW 2007, 2172 (2173). Ausführlich dazu W. Bolewski DVBl. 2007, $789 \mathrm{ff}$.

${ }^{157}$ Rechtlich unerheblich ist hingegen eine besondere Empfindlichkeit ausländischer Stellen gegenüber Kritik; dazu BVerfG (K), NJW 2007, 2167 (2169).

${ }^{158}$ Diese kann einen Einzelfall (Art. 25 Abs. 2) oder generelle Fragen (Abs. 3) betreffen. Völkerrechtliche Verträge können auch einen Vertragsschluss zwischen Behörden vorsehen. Ohne Grundlage im Völker- (oder im Gemeinschaftsrecht; dazu Niedobitek [Fn. 43], 144ff., 290ff.) kann ein verwaltungsrechtlicher grenzüberschreitender Vertrag (etwa zur rechtlichen Absicherung einer gemeinsamen Raumplanung) kaum zustande kommen. Er muss seine Grundlage in einer Rechtsordnung finden, und zumindest die deutsche sieht den Abschluss von Verträgen nach ausländischem öffentlichen Recht nicht vor; Niedobitek ebd., $324 \mathrm{ff} ., 414$.

159 Dazu M. Lehner in: Vogel/Lehner (Fn. 10), Art. 25 Rn 74f., 128.

160 Fastenrath (Fn. 61), 238; Engel (Fn. 68), 121.

${ }^{161}$ Dies ergibt sich schon aus Art. 19 Abs. 4 GG und Art. 6 EMRK; siehe dazu EGMR, Urteil vom 24. 11. 1994, 15287/89 - Beaumartin, §38; Urteil vom 13. 2. 2003, 49636/99 - Chevrol, $\$ \S 76 \mathrm{ff}$.

${ }_{162}$ Für individuelle Regelungen nach Art. 25 Abs. 2; Lehner(Fn. 159), Art. 25 Rn 75, 132; für generelle Verständigungen nach Abs. 3 ebd., Art. 25 Rn. 154; zum Sozialrecht BSGE 85, 240 (244); Glaser(Fn. 147), 102. Zum gleichen topos aus Sicht des IPR Schinkels (Fn. 147), $227 \mathrm{f}$. 
ein einzelner einen - gerichtlich überprüfbaren - Anspruch auf Einleitung eines solchen Verständigungsverfahrens. Zwar können außenpolitische Ermessensentscheidungen funktional kaum von Gerichten überprüft, es kann aber zumindest ein Ermessensmissbrauch ausgeschlossen werden. ${ }^{163}$

Umgekehrt wirft die Entsendung deutscher Verbindungsbeamter ins Ausland (vgl. etwa $\S 65$ Abs. 2 BPolG) zunächst die zu verneinende ${ }^{164}$ Frage nach der Erforderlichkeit einer gesetzlichen Grundlage auf. Eine Betätigung darf aber nur im Rahmen von nach deutschem Recht zulässigen Zwecken und unter Beachtung der Kernvorgaben des deutschen Rechts erfolgen.

\section{b) Amts- und Vollstreckungshilfe}

Nach der überzeugenden Entscheidung des BVerfG zum deutschösterreichischen Rechtshilfevertrag ${ }^{165}$, die unmittelbar die Vollstreckungshilfe betriff, aber für die Amtshilfe in gleicher Weise gilt, sind beide auch zugunsten des Auslands ${ }^{166}$ zulässig. Sie verlangt aber formal eine spezifische gesetzliche Regelung und materiell die Beachtung der Grundrechte. Für die deutsche Amtshandlung gelten diese in vollem Umfang. Mit Blick auf das ausländische Recht kann man dies, will man internationale Kooperation nicht unmöglich machen, nicht verlangen. $\mathrm{Zu}$ Recht lässt das BVerfG daher die Beachtung des deutschen ordre public, zu verstehen als Beachtung verfassungsrechtlicher Elementargarantien, ausreichen. ${ }^{167}$ Die sachliche Rechtfertigung findet sich regelmäßig

163 Dazu BFH, BStBI 1982 II 583 (586), unter Hinweis auf die bestehende, aber sachlich begrenzte Möglichkeit der gerichtlichen Kontrolle der Ausübung diplomatischen Schutzes (dazu BVerfGE 55, 349 [364] mit Kritik als unzureichend bei $K$. Hailbronner Kontrolle der auswärtigen Gewalt, VVDStRL 56 [1997], 7 [23]); Lehner (Fn. 159), Art. $25 \mathrm{Rn} .89 \mathrm{ff}$.

164 So auch Ohler (Fn. 12), 316.

165 B VerfGE 63, 363 (366). Grundsätzlich a.A. H.-J. Papier/B.-D. Olschewski DVB1. $1976,475 \mathrm{ff}$.

166 Wie bei der inländischen Amtshilfe ist zu unterscheiden zwischen der Ermächtigung zur Leistung (im Außenverhältnis), etwa einer Datenübermittlung ins Ausland, und zur Vornahme der Amtshandlung (im Innenverhältnis), etwa der Datenerhebung. Dazu W. Schomburg/O. Lagodny/S. Gleß/T. Hackner Internationale Rechtshilfe in Strafsachen, 4. Auflage 2006, Einleitung, Rn. $92 \mathrm{f}$.

${ }^{167}$ Im Zusammenhang mit auszuliefernden Ausländern wird sogar nur verlangt, dass im dortigen Staat rechtsstaatliche Elementargarantien eingehaiten werden; BVerfGE 63 , 332 (337); 75, 1 (16f.); 108, 129 (136); 113, 154 (162). Ganz in diesem Sinne überprüft auch der EGMR bei Ausweisungsentscheidungen nur, ob im anderen Staat eine mit Art. 3 EMRK als Fundamentalnorm unvereinbare Behandlung droht; die übrigen Garantien der EMRK spielen insoweit keine Rolle. Siehe grundlegend EGMR, Slg. A 161 
im Prinzip der Gegenseitigkeit, doch können im Einzelfall auch sonstige Erwägungen Amts- und Vollstreckungshilfe rechtfertigen. ${ }^{168}$ Zum bereits angesprochenen Datenaustausch bestehen verbreitet dem entsprechende Regelungen. ${ }^{169}$ Innerstaatlich ist die Vornahme einer Amtshandlung, je nach gesetzlicher Regelung direkt oder inzident auch die Leistung der Amtshilfe gerichtlich anfechtbar. ${ }^{170}$

Für die Vollstreckungshilfe gilt gleiches. Hinzu kommt die Frage, ob im Rahmen unproblematisch möglichen deutschen Rechtsschutzes gegen nationale Vollstreckungsakte inzident ausländische Rechtsakte am Maßstab des erwähnten ordre public überprüft werden können müssen. Weist die ausländische Rechtsordnung, vor allem der Rechtsschutz generell die notwendige Qualität auf, was bei den meisten Vertragsstaaten der EMRK in der Regel gegeben sein dürfte, ist dies verzichtbar. ${ }^{171}$ Deutsche Bitten um Amts- und Vollstreckungshilfe ins Ausland schließlich sind rechtlich unproblematisch, fordern insbesondere kein Gesetz. ${ }^{172}$

\section{Grenzüberschreitende Aktivitäten}

\section{a) Tätigkeit ausländischer Behörden im Inland}

In bemerkenswertem Umfang üben ausländische Behörden in Deutschland Hoheitsgewalt aus. So darf die Schweizer Polizei nach dem deutsch-schweizerischen Polizeiabkommen in Deutschland eine in der

\footnotetext{
$\S 86 \mathrm{ff}$. - Soering. Sind Personen betroffen, die dauerhaft unter dem Schutz der deutschen Rechtsordnung stehen, reicht das aber nicht aus; siehe Ohler (Fn. 12), 165.

${ }_{168}$ Im Recht der Doppelbesteuerung wird regelmäßig ein wechselseitiger Informationsaustausch auch insoweit vereinbart, wie es nicht nur um die Vermeidung von Doppelbesteuerung geht, sondern allein um die normale Steuererhebung. Dazu Engelschalk (Fn. 128), Art. 26 Rn. 4, 37; ferner Reimer (Fn. 10), 191. Zur Möglichkeit einer Klageerhebung gegen eine Datenweitergabe (allgemein dazu bei Fn. 130) Engelschalk, ebd., Rn. 74, 140. Die innerstaatliche Grundlage für die Datenerhebung (Vornahme) findet sich in $\S 117 \mathrm{AO}$, dort in Abs. 3 auch für die vertragslose Amtshilfe. Zu den verfassungsrechtlichen Grenzen siehe $S$. Eilers Das deutsche Steuergeheimnis als Grenze internationalen Auskunftsverkehrs 1987. Siehe ferner etwa $\$ 59$ IRG (i.V.m. $\$ 77$ als Verweis auf die Befugnisnormen für rein innerstaatliches Handeln), 44a Abs. 2 KWG, 19 Abs. 3 WpHG.

${ }^{169}$ Siehe Fn. $128 \mathrm{f}$.

${ }^{170}$ Dazu L. Harings Grenzüberschreitende Zusammenarbeit der Polizei- und Zollverwaltungen und Rechtsschutz in Deutschland, 1998, $217 \mathrm{ff}$., $222 \mathrm{f}$.

171 Siehe auch Art. 27 Abs. 8 OECD-Musterabkommen DBA und dazu Engelschalk (Fn. 128), Art. 26 Rn. 123.

172 Ohler (Fn. 12), 307. Rechtsschutz ist grundsätzlich nicht möglich; siehe BVerfGE 57, 9 (23); dazu Harings (Fn. 170), 211 ff, 216, 221 f. mwN.
} 
Schweiz begonnene Observation fortsetzen und Verdächtigen nacheilen, und zwar in dringenden Fällen auch ohne vorherige deutsche Zustimmung. ${ }^{173}$ Auf der Grundlage eines Abkommens zwischen RheinlandPfalz und Luxemburg ${ }^{174}$ haben Gemeinden beider Länder insgesamt drei öffentlich-rechtliche Wasserverbände gebildet, die gemeinsamer Aufsicht der beiden Länder unterstehen; Rechtsstreitigkeiten - mit Aufsichtsbehörden wie mit Bürgern - werden von einem gemeinsamen Schiedsgericht entschieden. ${ }^{175}$ Die Literatur hält trotzdem die Übertragung von Hoheitsrechten auf ausländische Staaten überwiegend für unzulässig; ${ }^{176}$ die einschlägige, zum Teil traditionsreiche Praxis - genannt seien auch gemeinsame Grenz- und Zollabfertigungsstellen - wird dann meist dadurch gerechtfertigt, dass bestimmte Fallkonstellationen nicht als Übertragung von Hoheitsrechten angesehen werden. ${ }^{177}$ Ehrlicher ist die gegenteilige Annahme; der Sperrwirkung von Art. 24 Abs. 1 GG ist durch enge Zulässigkeitsvoraussetzungen Rechnung zu tragen: beson-

173 BGBl. 2001 II 946, Art. 16 (Nacheile) und Art. 14f. (Observation); siehe ferner zur verdeckten Ermittlung Art. 17f. Möglichst vor oder bei Grenzübertritt hat jedoch eine entsprechende Mitteilung zu erfolgen. Auf Verlangen der Bundesrepublik ist der Einsatz einzustellen, Anweisungen sind zu befolgen. Ausführlich dazu Cremer ZaöRV 60 (2000), $103 \mathrm{ff}$.

174 Vertrag vom 7. Februar 1975, GVBl. Rheinland-Pfalz 1975, 54. Nach Angaben des Ministeriums für Umwelt, Forsten und Verbraucherschutz (Schreiben vom 11. 7. 2007) wurden auf dieser Grundlage die drei „Internationalen Abwasserklärwerke“ „Echternach-Weilerbach", „Rosport-Trier-Land" und „Mompach-Trier-Land" gegründet. Aus heutiger Sicht liegt hier wohl ein Fall von Art. 24 Abs. 1a GG vor; vgl. noch bei Fn. 181.

175 Zur rechtlichen Problematik sogleich bei Fn. 180f. - Einen weiteren, prominenten Fall bildet die Luftüberwachung im deutschen Südwesten durch die privatisierte Schweizer Flugverkehrsleitung Skyguide AG, vom LG Konstanz in seinem Urteil vom 27. 7. 2006 zum Flugzeugunglück in Überlingen am 1. 7.2002 alternativ als Fall der Beleihung oder der Unterbeleihung qualifiziert (zwischengeschaltet die Deutsche Flugsicherung); zudem hafte die Bundesrepublik für Fehlverhalten der Skyguide, da es Aufgabe der Bundesrepublik war, den deutschen Luftraum zu sichern. Dazu kritisch, mit entsprechenden Nachweisen, H.H. Rupp JZ 2006, 1033 ff.

176 Mosler HStR VII, § 175 Rn. 39; Randelzhofer in T. Maunz/G. Dürig, Grundgesetz, Art. 24 Abs. 1 Rn. 53; Rojahn (Fn. 61), Art. 24 Rn. 18; Ohler (Fn. 12), 143; Gramm DVB1. 1999, 1237; H.D. Jarass in: ders./B. Pieroth Grundgesetz, 9. Aufl. 2007, Art. 24 Rn. 7. Unproblematisch möglich ist dies aber im Anwendungsbereich des adressatenoffen formulierten Art. 23 GG.

177 Siehe M. Baldus Die Verwaltung 32 (1999), 481 (503): traditionell übliche Formen; ebenso Gramm ebd., 1238), und ähnlich Hecker AöR 127 (2002), 291 (311): Ausnahme für Marginalfälle; ferner ders., 305, sowie Niedobitek (Fn. 43), 431 f.: eine Hoheitsrechtsübertragung liege nur vor bei ausschließlichem Geltungsanspruch fremder Hoheitsakte, der vor allem bei faktischem Handeln ausländischer Behörden nicht vorliege; im Ergebnis ähnlich Cremer ebd., $133 \mathrm{f}$.: die Duldung fremder Hoheitsakte sei zulässig. 
dere Gründe müssen die Regelungen verlangen, die übertragenen Befugnisse müssen eng begrenzt sein. ${ }^{178}$

Zulässig ist es danach im Regelfall etwa, ausländischen Hoheitsträgern Rechte und Möglichkeiten einzuräumen, über die der Sache nach auch einfache Bürger verfügen. ${ }^{179}$ Dies gilt etwa für den Polizeivertrag: Observieren kann jedermann; für die Nacheile ist auf das allgemeine Festnahmerecht nach $\S 127$ StPO hinzuweisen. Die Wasserversorgung ist ohnehin nicht zwingend hoheitlich auszugestalten. ${ }^{180}$ Die Rechtsschutzgewähr schließlich ist elementare Konsequenz der Übertragung von Hoheitsrechten und kann kaum ohne ausländische Beteiligung realisiert werden. ${ }^{181}$

${ }^{178}$ Siehe Classen (Fn. 64), Art. 24 Rn. 65 ff.; ebenso Schmidt-ABmann FS R. Schmidt, 2006, 149 (161).

${ }^{179}$ Am hoheitlichen Charakter der entsprechenden Betätigung ändert dies aber nichts; dazu Baldus (Fn. 127), 496; Gramm DVBI. 1999, 1237 (1240). Ganz in diesem Sinne schreibt - für den umgekehrten Fall einer hoheitlichen Betätigung deutscher Stellen im Ausland - Art. 10 Abs. 2 des deutsch-niederländischen Zusatzabkommens zum NATO-Truppenstatut (BGBl. 1998 II 2407) vor, dass die deutschen Behörden Bestimmungen über den Schusswaffengebrauch durch deutsche Soldaten (nach deutschem Recht: nach $\$ 15 \mathrm{ff}$. UZwGBw) erlassen, die sich im Rahmen des niederländischen Notwehrrechts bewegen; dazu Ohler (Fn. 12), 80.

${ }^{180}$ Vergleichbar unproblematisch ist auch die Eröffnung der Möglichkeit, Zustellungen aus dem Ausland im Inland vorzunehmen. Siehe Art. 16 Abs. 2 des Abkommens über Soziale Sicherheit zwischen der Bundesrepublik Deutschland und Japan, BGBl. 1999 II 876, sowie Art. 16 Abs. 1 des entsprechenden Abkommens zwischen Deutschland und Korea, BGBl. 2001 II 915.

${ }^{181}$ In seiner Eurocontrol-Entscheidung problematisiert das BVerfG nicht einmal, dass hinsichtlich der gerichtlichen Kontrolle von Eurocontrol der entsprechende Vertrag (BGBl. 1962 II 2273) eine Zuständigkeit der belgischen Gerichte vorsah (BVerfGE 58, 1 [42]), obwohl insoweit gleichfalls eine Übertragung von Hoheitsrechten an einen ausländischen Staat vorgesehen war. In der Literatur wird die Arbeit von Eurocontrol insoweit (nach damaligem Recht) zum Teil als privatrechtlich qualifiziert mit der Folge, dass die entsprechenden Bestimmungen nur eine Regelung der internationalen Zuständigkeit enthielten und Art. 24 GG gar nicht einschlägig war (so C. Tomuschat BK, GG, Art. 24 Rn. 112). - Zur Möglichkeit des Rechtsschutzes durch ausländische Gerichte auch Kotzur (Fn. 43), 551, 555. Im Anwendungsbereich von Art. 24 Abs. 1a GG ist danach eine entsprechende Regelung auch durch Landesgesetz möglich, wie in dem bei Fn. 174 erwähnten, vor Schaffung dieser Norm abgeschlossenen (und daher nach h.M., nicht nach hier vertretener Auffassung wohl als verfassungswidrig anzusehenden) Abkommen zwischen Luxemburg und Rheinland-Pfalz der Fall; tendenziell aA $U$. Beyerlin ZaöRV 44 (1994), 587 (598); an der Zulässigkeit dieser Rechtsschutzregelung zweifelnd auch $H$. Heberlein DöV 1996, 100 (106). In diesem Lichte lassen sich wohl auch die von mir früher (Fn. 64, Art. 24, Rn. 75) skizzierten Probleme der Rechtsschutzgewähr im Anwendungsbereich von Art. 24 Abs. 1a GG bewältigen. 
Bei der Tätigkeit im Inland sind ausländische Behörden im Regelfall an das „allgemeine“ inländische Recht gebunden. ${ }^{182}$ Eine Bindung an das inländische öffentliche Recht wirft die bereits erwähnten Probleme bei der Trennbarkeit von materiellem und Verfahrens-, vor allem Organisationsrecht auf ${ }^{183}$ und sollte daher die Ausnahme bleiben. ${ }^{184}$ Die Gewährleistung von Rechtsschutz ist rechtsstaatlich zwingend, sollte aber aus dem soeben genannten Grund durch Gerichte des Herkunftsstaates erfolgen. ${ }^{185}$ Hierfür spricht auch, dass die gerichtliche Kontrolle der Verwaltung in ein von Staat zu Staat divergierendes Verständnis von Gewaltenteilung eingebettet ist. ${ }^{186}$ Etwas einfacher sind die Dinge bei der Haf-

182 Ohler (Fn. 12), 69 ff.

183 Siehe oben bei Fn. 151.

184 Ohler (Fn. 12), 72 ff. Der dt.-schweiz. Polizeivertrag sieht demgegenüber eine Bindung grenzüberschreitender Aktivitäten auch an das Recht des Staates, auf dessen Gebiet die Beamten tätig werden, vor (Art. 14 Abs. 3 Nr. 1, Art. 16 Abs. 4 Nr. 1). Die Bindung an das Recht des Herkunftsstaates bleibt davon unberührt (Harings [Fn. 170] 227f.; aA Baldus [Fn. 127] 338).

185 So auch für den dt.-schweiz. Polizeivertrag Cremer ZaöRV 60 (2000), 103 (138). In der in der vorigen Fn. genannten Konstellation müssen die Schweizer Gerichte dann aber auch deutsches Recht anwenden (Harings [Fn. 170], 283). Da es hier grundsätzlich um Realakte geht - die jeweiligen Entscheidungen der Verwaltungen finden, da es jeweils nur um die Fortsetzung von Observation und Nacheile geht, im Ausland statt -, verbindet sich damit immerhin nicht die Notwendigkeit, Rechtsakte am Maßstab ausländischen Rechts zu messen, bei der die erwähnten Probleme wohl größere Bedeutung haben als bei faktischem Handeln. - Daneben kann in Deutschland gegen die deutschen Stellen geklagt werden auf Ausübung des jeweils vorgesehenen Weisungsrechts nach den in Fn. 173 genannten Normen; Harings ebd., 289ff. In einigen, aber (bis heute) nicht allen Bundesländern wird das Handeln ausländischer Polizeibeamter der zuständigen inländischen Polizeidienstelle zugerechnet; dazu Harings ebd., 283.

186 Besonders deutlich zeigt sich dies bei der unterschiedlichen Dichte der gerichtlichen Kontrolle der Verwaltung; zu unterschiedlichen Konzeptionen im internationalen Vergleich etwa $J$. A. Frowein (Hrsg.) Die Kontrolldichte bei der gerichtlichen Überprüfung der Verwaltung, 1993; Classen (Fn. 94), 119ff.; vgl. auch Nolte (Fn. 1), III 3; aA Dutta (Fn. 148), 411 f. Konkret: Nach Art. 34 Abs. 2 des erwähnten deutsch-niederländischen Zusatzabkommens (Fn. 179) können auf Ersuchen statt der an sich anwendbaren niederländischen auch deutsche Vorschriften Anwendung finden, ,soweit diese auf dem Gebiet der öffentlichen Sicherheit und Ordnung im Einzelfall höhere Anforderungen stellen als die niederländischen Vorschriften." Wenn Streit um die Frage entsteht, ob die Voraussetzungen der Norm einschlägig sind und auf wessen Beurteilung es ankommt, ist unklar, ob die Frage, ob und inwieweit das deutsche Baurecht Beurteilungsspielräume zugunsten der Verwaltung kennt, im hier relevanten Sinne materiellrechtlichen Charakter hat - dann gelten deutsche Maßstäbe, nach denen das Gericht entscheidet - oder prozessualen Charakter - dann gelten niederländische Maßstäbe mit der Folge, dass eventuell die Einschätzung der Verwaltung kaum hinterfragt werden kann. Die Beantwortung der Frage hängt eng mit dem Verständnis des Beurteilungsspielraums insgesamt zusammen. 
tung; hier wirft eine Zurechnung ausländischen Handelns an einen deutschen Hoheitsträger weniger Probleme auf. ${ }^{187}$

\section{b) Tätigkeit inländischer Behörden im Ausland}

SchlieBlich nehmen auch umgekehrt deutsche Stellen als solche Aufgaben im Ausland wahr. Spiegelbildlich zum vorstehend Dargestellten kommt dies vor, wenn deutsche Behörden in Nachbarstaaten tätig werden, ferner im Rahmen internationaler Aufbauhilfe (vgl. auch $\S 8,64$ Abs. 4 BPolG). Dabei überzeugt es nicht, nach der territorialen Reichweite der entsprechenden Befugnisnormen zu fragen. Entscheidend sind die Aufgaben. Decken diese einen Einsatz im Ausland ab, gelten für diesen, soweit nichts anderes geregelt ${ }^{188}$, die normalen Befugnisnormen..$^{189}$ Diese sollen ja die Erfüllung (aller) Aufgaben ermöglichen. Deckt die Aufgabenbeschreibung einen Auslandseinsatz nicht ab, erfordert ein solcher schon wegen der Bindung der Verwaltung an das Gesetz eine besondere gesetzliche Grundlage. Diese ist ggf. im Zustimmungsgesetz zu einem entsprechenden Vertrag zu sehen. Die Bindung an einschlägige internationale Menschenrechte bleibt ohnehin im Grundsatz unberührt. 190

\section{Die Aufgabe der Rechtswissenschaft}

Die zahlreichen Beispiele aus den unterschiedlichen Bereichen der Verwaltung haben das Ausmaß und die Formenvielfalt ihrer Internationalisierung deutlich gemacht. Gestern wurde gefragt, ob die Globalisie-

187 So etwa auch Art. 32 Abs. 4 des erwähnten dt-schweiz. Polizeivertrages.

188 Baldus (Fn. 127), 240 ff.

189 D. Kugelmann Die Kriminalpolizei 2007, 8 (12f.); Baldus (Fn. 127), 243, 334; Schorkopf(Fn. 84), § 7 II 2; vgl. auch Ohler (Fn. 12), $78 \mathrm{ff}$, $316 \mathrm{ff}$. - Eine (unmittelbare) Anwendung ausländischen Rechts durch deutsche Behörden stößt auf die gleichen Probleme wie die Anwenđung deutschen Rechts durch ausländische Behörden (dazu bei Fn. $147 \mathrm{ff}$.) und dürfte daher im wesentlichen nur in Betracht kommen, wenn deutsche Stellen - etwa im Rahmen von Aufbauhilfe und Ähnlichem - zeitlich begrenzt funktional als ausländische Behörden tätig werden.

190 Demgegenüber ist nach Auffassung des EGMR die EMRK als Instrument regionalen Menschenrechtsschutzes schon nach ihrem Selbstverständnis nicht auf MaBnahmen anwendbar, die ihre rechtliche Grundlage in Entscheidungen des UN-Sicherheitsrates finden. Siehe dazu (mit entsprechender Kritik) in Fn. 101. Ggf. ist auch die begrenzte Wirkungskraft von Grundrechten bei Sachverhalten mit Auslandsberührung zu bedenken; dazu mit unterschiedlichen Akzenten Nolte (Fn. 1), II 3; Poscher (Fn. 70), $190 \mathrm{ff}$; P. Badura in: D. Merten/H.-J. Papier (Hrsg.) Handbuch der Grundrechte, Band II, $2006 \S 47$ mwN.; abl. Baldus (Fn. 127), $234 \mathrm{ff}$. 
rung ein neues Phänomen ist; offene Grenzen hat es ja auch schon in der zweiten Hälfte des 19. und zu Beginn des 20. Jahrhunderts gegeben. Heute aber sind wesentlich mehr Lebensbereiche als damals stark verrechtlicht, so dass heute auch die Rechtsordnung und die sie ausführende Verwaltung wesentlich intensiver als früher von der Internationalisierung betroffen sind.

Im Einzelnen konnten nur einige grobe Strukturen angesprochen werden. Die Rechtswissenschaft ist aufgerufen, im Lichte der Erfahrungen, die in bestimmten Rechtsgebieten wie traditionell vor allem im Steuerrecht oder in den letzten Jahren verstärkt im Polizeirecht gemacht wurden, für andere Rechtsgebiete - selbstverständlich unter Berücksichtigung der jeweiligen Besonderheiten - Lösungen zu erarbeiten; der hier entfaltete Problemkatalog kann dafür eine Grundlage bilden.

Für individualbezogene internationale Entscheidungen, mit Vorsicht auch für die verfahrensmäßige Stellung der Staaten gegenüber internationaler Verwaltung müssen diese Lösungen vor allem auf internationaler Ebene gefunden werden. Insoweit ist die Wissenschaft in Art. 38 Abs. 1 lit. d IGH-Statut als „Hilfsmittel zur Feststellung von Völkerrechtssätzen“ sogar ausdrücklich anerkannt. Wichtig ist sie vor allem bei der Herausbildung von allgemeinen Rechtsgrundsätzen, da sich diese auf rechtsvergleichende Arbeiten stützen muss. Zudem kann die Wissenschaft die in der Vielzahl von Organisationen gemachten Erfahrungen zusammenführen und damit den Beitrag zur Einheitlichkeit (und damit Rationalität) der Rechtsanwendung leisten, ${ }^{191}$ den auf nationaler Ebene oberste Gerichte bewirken können. Allgemeine Rechtsgrundsätze dürfen jedoch „nicht als Einbruchstelle für zwar Wünschenswertes, aber eben nicht Wirkliches dienen."192 Ihre Heranziehung setzt die methodengerechte Feststellung von Lücken im Völkerrecht ${ }^{193}$ voraus.

Im Übrigen, vor allem mit Blick auf die Übernahme internationaler Standards und Empfehlungen sowie die zwischenstaatlichen Kooperationen, ist die Internationalisierung der Verwaltung anders als deren $\mathrm{Eu}-$ ropäisierung formal stark vom nationalen Recht abhängig. ${ }^{194}$ Dieses

191 Dazu auch $M$. Sørensen ICLQ 32 (1983), 559 (563); Schermers/Blokker (Fn. 19), 3 27; Amerasinghe ICLQ 45 (1996), 773 (778). Zur Rolle der (Staatsrechts-)Wissenschaft in diesem Zusammenhang (bezogen auf das BVerfG) $H$. Schulze-Fielizz in: ders. (Fn. 117), 11 (29).

192 W. Graf Vitzthum in ders. (Hrsg.), Völkerrecht, 3. Aufl. 2004, 1. Abschn. Rn. 143 Fn. 358. In der 4. Auft. (Fn. 65) ist die gleiche Aussage ebd., jetzt Fn. 359, etwas knapper gefasst.

${ }^{193}$ Dazu U. Fastenrath Lücken im Völkerrecht, 1991, insbesondere $212 \mathrm{ff}$.

194 Der Einfluss der EMRK und damit des EGMR auf die Entwicklung des Verwaltungsrechts ist zwar nicht unerheblich; er beschränkt sich aber im Wesentlichen auf 
aber wird inhaltlich nicht selten von ausländischen oder internationalen Rechtsgütern oder gar Hoheitsakten mitbestimmt. Daher sind rechtsvergleichende Untersuchungen auch hier durchaus hilfreich. ${ }^{195}$ Angesichts der Tatsache, dass völkerrechtliche Verpflichtungen regelmäßig ergebnisbezogenen Charakter aufweisen, nationale verwaltungsrechtliche Normen aber regelmäßig handlungsorientierten Charakter aufweisen, können ggf. auch die heute Vormittag angestellten Überlegungen dazu beitragen, im Rahmen der gebotenen völkerrechtskonformen Auslegung des nationalen Rechts sicherzustellen, dass die eingegangenen Verpflichtungen auch tatsächlich erfüllt werden. Nationale Instrumente können ggf. auch Kompensationsfunktion übernehmen. In der Sache muss auf mehreren Ebenen ein Ausgleich gefunden werden: institutionell zwischen der demokratischen Eigenständigkeit staatlicher Entscheidung 196 und der Eingliederung des Staates in die Völkerrechtsgemeinschaft, materiell- und verfahrensrechtlich zwischen den eigenen Standards und dem Respekt vor fremden Rechtskulturen. Dabei ist zu beachten, dass ausländische und internationale Rechtslagen nicht zur Disposition des deutschen Gesetzgebers stehen; der berühmte „schonende" Ausgleich kann insoweit also nur in Grenzen praktiziert werden. Auch ist ein Unterschied zwischen vertikaler und horizontaler Internationalisierung zu beachten: Erstere ist durch eine wechselseitige institutionelle und materiellrechtliche Verflechtung gekennzeichnet. Bei letzterer hingegen muss es zwar eine wechselseitige Abstimmung und Koordination geben; zumindest bei außenwirksamem Handeln aber müssen Rechtsordnung und Akteure ihren eigenständigen Charakter behalten.

Möglicherweise liegen die eigentlichen Probleme aber ohnehin vor allem beim gestern diskutierten verfassungsrechtlichen Rahmen. Hinsichtlich der verwaltungsrechtlichen Instrumente kann man nämlich

rechtsstaatliche Verfahrensgarantien und bleibt daher gegenständlich begrenzt. Einen Ausnahmefall bildet das Ausländerrecht; dazu etwa J. Bast in: Möllers/VoBkuhle/Walter (Fn. 5), 277 (297ff.).

${ }^{195}$ Dies gilt insbesondere dann, wenn - notwendigerweise - offene verfassungsrechtliche Vorgaben wie Demokratie- und Rechtsstaatsprinzip sowie die Grundrechte zu konkretisieren sind; dazu allgemein I. Pernice in: H. Schulze-Fielitz (Fn. 117), 225 (239).

196 Zur Einbeziehung der "offenen Staatlichkeit" in das Demokratieverständnis BVerfGE 63, 343 (370); Cremer ZaöRV 60 (2000), 103 (138f.); Hecker AöR 127 (2002), 291 (319ff.); Schinkels (Fn. 147), $205 \mathrm{ff}$. In Erinnerung ist insbesondere zu rufen, dass die im Rahmen des IPR erfolgende, vom BVerfG (E 31, 58 [77]) implizit gebilligte Heranziehung ausländischen Rechts unzulässig wäre, wollte man die für inländisches Recht geltenden, aus dem Demokratieprinzip abgeleiteten Anforderungen hier in gleicher Weise anwenden. 
vielleicht auf Antworten zurückgreifen, die für die Landesverwaltungen im Rahmen der föderalen Struktur der deutschen Verwaltung gefunden wurden: hier müssen Vorgaben des Bundes verarbeitet, es muss mit Kooperationswünschen und Verwaltungsakten anderer Länder umgegangen werden. ${ }^{197}$ Ein wirklich ,internationales“ Verwaltungsrecht jedenfalls kann es nur in gewissen Grenzen geben. ${ }^{198}$

197 So auch die Einschätzung von $M$. Ruffert Die Globalisierung als Herausforderung an das Öffentliche Recht 2004, 68.

198 Soweit es um die erwähnte Beeinflussung des nationalen Rechts geht, ist der Begriff der "Internationalisierung“ vielleicht eher angebracht. 
Leitsätze des 1. Berichterstatters über:

\section{Die Entwicklung eines Internationalen Verwaltungsrechts als Aufgabe der Rechtswissenschaft}

\section{Einführung}

1. Die Internationalisierung der Verwaltung, deren rechtliche Rahmenbedingungen hier als „Internationales Verwaltungsrecht" verstanden werden, wirft insbesondere Fragen auf nach Legitimation und rechtsstaatlicher Qualität des Verwaltungshandelns, nach Rechtsschutz und Haftung.

\section{II. „Vertikale“ Internationalisierung der Verwaltung}

2. Internationale Organisationen und sonstige Institutionen (einschließlich informeller Gremien) nehmen Verwaltungsaufgaben (neben der internen Personalverwaltung) vor allem wahr:

- bei territorial definierten Sonderregimen;

- bei der Bewirtschaftung knapper Ressourcen;

- bei der Informationssammlung und -auswertung (vor allem im Zusammenhang mit einschlägigen materiellen Verpflichtungen);

- bei der Gewinnung von Sachverstand und darauf aufbauend der Erarbeitung von Handlungsempfehlungen, insbesondere von - rechtlich verbindlichen oder zumindest praktisch stark durchsetzungsmächtigen Standards; damit wird nicht zuletzt ein Ausgleich für die mit der Globalisierung verbundene Öffnung von Grenzen geschaffen.

3. Für die Stellung der Staaten gegenüber internationaler Verwaltung sind (nur als ius cogens denkbare) verbindliche rechtsstaatliche Standards kaum zu entwickeln. Es lassen sich aber allgemeine Rechtsgrundsätze (Art. 38 Abs. 1 lit. c IGH-Statut) herausbilden, vor allem als Leitlinien für das Verfahrensrecht, soweit dies für eine sachgerechte Rechtsanwendung geboten ist.

4. Eine Außenvertretung in internationalen Institutionen durch nachgeordnete Behörden verlangt entsprechende interne Koordinationsmechanismen. Innerhalb der Bundesregierung kommt das Ministerialprinzip an Grenzen. 
5. Von Internationalen Organisationen beschlossene, verbindliche Sekundärrechtsakte sind bei der Auslegung und Anwendung deutschen Rechts zu berücksichtigen. Empfehlungen sollen dabei in Abhängigkeit vom jeweiligen institutionellen Rahmen, der Art ihres Zustandekommens und ihrer inhaltlichen Qualität herangezogen werden, bei sonstigen Absprachen ist dies möglich. Unmittelbare Rechte und Pflichten für den Einzelnen können nur durch einen besonderen Anwendungsbefehl begründet werden. In den bekannten engen Grenzen ist auch ein dynamischer Verweis zulässig.

6. Die Beeinflussung deutschen Verwaltungshandelns durch nicht-deutsche Stellen stellt im Lichte des Demokratieprinzips kein prinzipielles Problem dar. Die Exekutivlastigkeit internationaler Kooperation ist verfassungsrechtlich verankert. Vor allem aber dient die Öffnung des Staates inhaltlich verfassungsrechtlich verankerten Zielen: dem Frieden, rechtlich gesicherter grenzüberschreitender Freiheitsentfaltung und der Verbesserung der Qualität staatlicher Entscheidungen. Zudem wirken ggf. auf nationaler Ebene zu erfüllende Informationspfichten kompensatorisch.

7. Bei Verweisungen reicht eine internationale Veröffentlichung. Diese kann in Englisch erfolgen, wenn von den Adressaten dessen Kenntnis verlangt wird.

8. Einer gerichtlichen Kontrolle internationaler Standards sind Grenzen gesetzt: Nach den völkerrechtlichen Auslegungsregeln kommt auch der einschlägigen Staatenpraxis Bedeutung zu. Inhaltlich sind funktionalrechtliche Grenzen der gerichtlichen Kontrolle einschlägiger Regelwerke sowie ggf. die Pflicht zur Berücksichtigung völkerrechtlicher Verpflichtungen (These 5) zu beachten.

9. Bei individualbezogenem Handeln internationaler Organisationen sind die Menschenrechte als Gewohnheitsrecht und - Lücken füllend - allgemeine Rechtsgrundsätze (Art. 38 Abs. 1 lit. c IGH-Statut) als Maßstäbe zu beachten.

10. Soweit international kein Rechtsschutz bereitsteht, stehen die völkerrechtlichen Verpflichtungen ggf. unter einem menschenrechtlich begründeten Rechtsschutzvorbehalt zugunsten zuständiger nationaler Gerichte.

11. Der Informationsaustausch mit internationalen Stellen verlangt eine gesetzliche Regelung. Voraussetzung ist vergleichbarer Datenschutz oder die Gefährdung von Elementargütern (vgl. auch § 4 b BDSG). Die Nutzung von im Ausland gewonnenen Informationen setzt deren rechtsstaatliche Qualität voraus. 


\section{Horizontale Internationalisierung der Verwaltung}

12. Die Einbeziehung ausländischer Schutzgüter in deutsche Normen wird teilweise von Art. 26 (iVm Art. 1 Abs. 2) GG und dem Völkerrecht verlangt; im Übrigen ist es kraft gesetzgeberischer Entscheidung zulässig.

13. Deutsche Normen können auch an ausländische Rechtslagen anknüpfen. Im Rahmen von Rechtsstreitigkeiten vor deutschen Gerichten können diese idR nicht inzident überprüft werden. Ausländisches Recht kann grundsätzlich keinen Maßstab für das Handeln deutscher Behörden darstellen.

14. Die Abstimmung von deutschen Verwaltungsentscheidungen mit ausländischen Stellen setzt grundsätzlich die volle Überzeugung der deutschen Behörden von deren Rechtmäßigkeit oder eine entsprechende gesetzliche/ vertragliche Regelung voraus; eine Bindung der Gerichte wird in keinem Fall herbeigeführt.

15. Amts-/Vollstreckungshilfe zugunsten ausländischer Staaten ist aufgesetzlicher Grundlage zulässig, aber an einen am ordre public ausgerichteten Rechtsschutz durch deutsche Gerichte gekoppelt, soweit dieser im Ausland nicht adäquat gewährleistet ist.

16. Die Übertragung von Hoheitsrechten auf ausländische Staaten ist in begrenztem Ausmaß zulässig (etwa die Observations- und Nacheilerechte nach dem dt.-schweiz. Polizeivertrag). Handeln ausländische Behörden am Maßstab des Heimatrechts, ist eine angemessene gerichtliche Kontrolle nur durch die Heimatgerichte möglich; bei der Haftung wirft eine Zurechnung an nationale Stellen keine Probleme auf.

17. Beim Handeln deutscher Stellen im Ausland kommt für den Binnenbereich deutsches Recht uneingeschränkt zur Anwendung, im Außenverhältnis nur, soweit der zugrunde liegende Auftrag nichts anderes vorgibt. Internationale Menschenrechte bleiben im Kern verbindlich.

\section{Die Aufgabe der Rechtswissenschaft}

18. Im Lichte bereichsspezifischer Erfahrungen muss die Rechtswissenschaft Lösungen für die aus der Internationalisierung der Verwaltung resultierenden Probleme entwickeln.

19. Diese sind für die Stellung der Staaten gegenüber internationaler Verwaltung sowie für internationale individualbezogene Entscheidungen vorwiegend im Völkerrecht zu suchen. Die Wissenschaft wirkt, vor allem im Zusammenhang mit der hierfür notwendigen Rechtsvergleichung, „als Hilfsmittel zur Feststellung von Rechtsnormen " (Art. 38 Abs. I lit. d IGH-Statut).

20. Im Übrigen ist nationales Recht maßgeblich, das auch eine Kompensationsfunktion übernehmen kann. Dessen Inhalte sind jedoch ggf. auslän- 
disch/international mitgeprägt. Institutionell muss vor allem ein Ausgleich zwischen der demokratischen Eigenständigkeit staatlicher Entscheidung und der Eingliederung des Staates in die Völkerrechtsgemeinschaft gefunden werden, materiell- und verfahrensrechtlich der zwischen den eigenen Standards und dem Respekt vor fremden Rechtskulturen. 
Vierter Beratungsgegenstand:

\section{Die Entwicklung eines Internationalen Verwaltungsrechts als Aufgabe der Rechtswissenschaft}

2. Bericht von Professor Dr. Giovanni Biaggini, Zürich

Inhalt

Seite

I. „Internationales Verwaltungsrecht“ als Phänomen . . . . . 414

1. Ausgangspunkte und Herangehensweisen . . . . . . 414

2. Begriffliche Klärungen . . . . . . . . . . . . 416

a. Überkommener Begriffsgebrauch . . . . . . . . . 416

b. Problemorientierter Ansatz . . . . . . . . . . 418

II. Grundprobleme eines „Internationalen Verwaltungsrechts“ . 421

1. Suche nach einem Ordnungsrahmen . . . . . . . . 421

2. Strukturen der Rechtsverwirklichung im Bereich des

Verwaltungsrechts . . . . . . . . . . . . 424

3. Folgerungen und Folgefragen . . . . . . . . . . . 427

III. Grundfragen eines „Internationalen Verwaltungsrechts“ . . 427

1. Zentrale und dezentrale Rechtsverwirklichung als

Grundmodelle . . . . . . . . . . . . . . . . 427

2. Gemeinsamkeiten und Unterschiede . . . . . . . . . 429

3. Ansätze zum Ausgleich des strukturellen Defizits in der völkerrechtlichen Praxis . . . . . . . . . . . . . 433

IV. Perspektiven . . . . . . . . . . . . . . 437

1. Aufgaben der Rechtswissenschaft . . . . . . . . 437

2. Zur Einordnung des „Internationalen Verwaltungsrechts" . . . . . . . . . . . . 438 


\section{I. „Internationales Verwaltungsrecht“ als Phänomen}

\section{Ausgangspunkte und Herangehensweisen}

Die staatliche Verwaltung und das Verwaltungsrecht haben sich schon immer mit grenzüberschreitenden Fragen und Vorgängen beschäftigt. ${ }^{1}$ Die Bedeutung der internationalen Dimension des Verwaltens nimmt jedoch in jüngerer Zeit, jedenfalls in Europa, beträchtlich zu, dies weit über das traditionell international geprägte Wirtschaftsrecht hinaus ${ }^{2}$ und - wie man aus Schweizer Sicht ergänzen kann - unabhängig davon, ob EU-Mitgliedstaat oder nicht. ${ }^{3}$ Die Gründe dafür sind vielfailtig, die Auswirkungen nicht minder. In der Literatur spricht man bildhaft von einem Zusammenrücken von Völkerrecht und Verwaltungsrecht. ${ }^{4}$ In der Tat: Das Völkerrecht zeigt inhaltlich vermehrt verwaltungsrechtliche Züge, das Verwaltungsrecht hat zunehmend völkerrechtliche Hinter-

\footnotetext{
${ }^{1}$ Vgl. Lorenz von Stein Einige Bemerkungen über das internationale Verwaltungsrecht, in: Jahrbuch für Gesetzgebung, Verwaltung und Volkswirtschaft im Deutschen Reich 6 (1882), 395; Johann Caspar Bluntschli Die Organisation des europäischen Staatenvereins, in: ders., Gesammelte kleine Schriften, Band II, 1881, 279 ff.; Ernst Isay Internationales Verwaltungsrecht, in: Fritz Stier-Somlo/Alexander Elster (Hrsg.), Handwörterbuch der Rechtswissenschaft, Band 3, 1928, 344; Eberhard Menzel Nationale und internationale Verwaltung, DÖV 1969, 1. Weitere Hinweise bei Christian Tietje Internationalisiertes Verwaltungshandeln, 2001, $116 \mathrm{ff}$;; Miloš Vec Recht und Normierung in der Industriellen Revolution, 2006, $126 \mathrm{ff}$.

2 Vgl. den Beitrag von Claus Dieter Classen in diesem Band sowie (aus verwaltungsbzw. politikwissenschaftlicher Sicht) Klaus König Öffentliche Verwaltung und Globalisierung, Verwaltungsarchiv 92 (2001), 475; Wolfgang Wessels Die Öffnung des Staates Modelle und Wirklichkeit grenzüberschreitender Verwaltungspraxis (1960-1995), 2000; Laurent Goetschel u.a., Schweizerische Aussenpolitik, 2002, $67 \mathrm{ff}$.

${ }^{3}$ Vgl. etwa Jean-Bernard Auby La globalisation, le droit et l'Etat, 2003; Sabino Cassese Lo spazio giuridico globale, 2003; Heinrich Koller Globalisierung und Internationalisierung des Wirtschaftsrechts, Zeitschrift für Schweizerisches Recht 119 (2000) II, $313 \mathrm{ff}$; Matthias Ruffert Die Globalisierung als Herausforderung an das Öffentliche Recht, 2004.

4 Vgl. Eberhard Schmidt-ABmann Die Herausforderung der Verwaltungsrechtswissenschaft durch die Internationalisierung der Verwaltungsbeziehungen, Der Staat 45 (2006), 315 (316); vgl. auch Nico Krisch/Benedict Kingsbury Global Governance and Global Administrative Law in the International Legal Order, European Journal of International Law 17 (2006), 1 (11); Christoph Möllers Internationales Verwaltungsrecht, in: ders./Andreas Voßkuhle/Christian Walter (Hrsg.), Internationales Verwaltungsrecht, 2007, 1 (2) („Annäherungen von Völkerrecht und Verwaltungsrecht“); Christian Tietje The Changing Legal Structure of International Treaties as an Aspect of an Emerging Global Governance Architecture, in: German Yearbook of International Law 42 (2000), 26 (35).
} 
gründe - ein Umstand, der im EU-Raum mitunter durch das Gemeinschaftsrecht verdeckt wird. ${ }^{5}$

Es wäre gewiss reizvoll, das Internationalisierungsphänomen und seine Konsequenzen aus Schweizer Sicht zu beleuchten - in Parallele zum Referat von Herrn Classen. Ich möchte indes anders vorgehen und den Schwerpunkt nicht bei den vielfältigen Einzelfragen der „vertikalen“ und ,horizontalen“ Internationalisierung setzen, sondern mich in erster Linie den internationalen Aspekten des Verwaltungs rechts - als rechtswissenschaftliche Disziplin - zuwenden. Dabei rücken konzeptionelle und strukturelle Fragen in den Vordergrund. Auf die im Rahmen der Tagung bereits mehrfach angesprochenen Themen Rechtsschutz und demokratische Legitimation werde ich nicht vertieft eingehen.

An Beschreibungen des Internationalisierungsphänomens herrscht heute kein Mangel. Das Thema war in jüngerer Zeit, nicht nur im deutschen Sprachraum, Gegenstand von Monografien und Aufsätzen, Symposien und Werkstattgesprächen und daraus hervorgehenden Veröffentlichungen. ${ }^{6}$ Zwei der vier für den Schweizerischen Juristentag 2006 verfassten Berichte standen unter dem Titel „Internationales Verwaltungsrecht $\%$,"Droit administratif international ".

Eine weitere Beschreibung des Phänomens wird im Folgenden nicht angestrebt. Die Aufmerksamkeit soll vielmehr der Frage gelten, wie die Rechtswissenschaft auf das Phänomen reagieren soll. In Betracht kommen zwei Möglichkeiten. Man kann die internationale Dimension des Verwaltens entweder in bestehende rechtswissenschaftliche Disziplinen integrieren (allenfalls als neue Unterdisziplin ${ }^{8}$ ) oder aber verselbstständi-

${ }^{5}$ Zum "Gemeinschaftsrecht als Medium der Internationalisierung“ vgl. Reiner Schmidt Die Internationalisierung des öffentlichen Wirtschaftsrechts, in: Die Wissenschaft vom Verwaltungsrecht, Die Verwaltung, Beiheft 2, 1999, 165 (166).

6 Vgl. Stefano Battini Amministrazioni senza stato. Profili di diritto amministrativo internazionale, 2003; Franz C. Mayer Die Internationalisierung des Verwaltungsrechts, Hab.schr., 2005 (i.E.); Christoph Ohler Die Kollisionsordnung des Allgemeinen Verwaltungsrechts - Strukturen des Internationalen Verwaltungsrechts, 2005; Christian Tietje Internationalisiertes Verwaltungshandeln, 2001; Benedict Kingsbury/Nico Krisch/Richard B. Stewart/Jonathan B. Wiener (eds.) The Emergence of Global Administrative Law, Law and Contemporary Problems 68 (2005), Nr. 3-4; Nico Krisch/Benedict Kingsbury (eds.) Symposium: Global Governance and Global Administrative Law in the International Legal Order, European Journal of International Law 17 (2006) Nr. 1; Christoph Möllers/ Andreas VoBkuhle/Christian Walter (Hrsg.) Internationales Verwaltungsrecht, 2007.

${ }^{7}$ Christine Breining-Kaufmann Internationales Verwaltungsrecht, Zeitschrift für Schweizerisches Recht 125 (2006) II, 5 ff.; Minh Son Nguyen Droit administratif international, Zeitschrift für Schweizerisches Recht 125 (2006) II, $75 \mathrm{ff}$.

${ }^{8}$ Beispiele: Internationales Steuerrecht als Teildisziplin des Steuerrechts, Internationales Sozial(verwaltungs)recht als Unterdisziplin des Sozialrechts. 
gen, wie dies etwa beim Internationalen Wirtschaftsrecht geschehen ist. ${ }^{9}$ In der Literatur deuten sich Verselbstständigungstendenzen an..$^{10}$

$\mathrm{Da}$ es unserem Berufsstand nicht vergönnt ist, durch grosse Erfindungen oder Entdeckungen in die Annalen der Wissenschaft einzugehen, mag der Reiz umso grösser sein, eine Pionierrolle bei der Lancierung und Etablierung eines neuen Rechtsgebiets zu spielen. Ist das „Internationale Verwaltungsrecht" ein taugliches Objekt dafür? Auf die Frage nach Einordnung und Stellenwert des „Internationalen Verwaltungsrechts “ im Gefüge der öffentlich-rechtlichen Disziplinen wird zurückzukommen sein (IV.).

\section{Begriffliche Klärungen \\ a. Überkommener Begriffsgebrauch}

Zuvor sind einige begriffliche Klärungen erforderlich. Die internationale Dimension des Verwaltens ist nicht nur der Sache nach, sondern auch dem Begriff nach im rechtswissenschaftlichen Diskurs schon länger präsent. Mehr als andere Rechtssprachen sieht sich die deutsche Rechtssprache beim „Internationalen Verwaltungsrecht" mit Mehrdeutigkeiten konfrontiert." In der italienischen Rechtssprache etwa stehen zwei Begriffe zur Verfügung: diritto amministrativo internazionale und (mit umgekehrter Adjektiv-Abfolge) diritto internazionale amministrativo - wobei man sich über die Tragweite der Wortfolge nicht ganz einig ist. ${ }^{12}$ Diese Möglichkeit der Differenzierung besteht auch in der franzö-

9 Vgl. z. B. Matthias Herdegen Internationales Wirtschaftsrecht, 6. Aufl. 2007.

${ }^{10} \mathrm{Vgl}$. z.B. Benedict Kingsbury/Nico Krisch/Richard B. Stewart The Emergence of Global Administrative Law, Law and Contemporary Problems 68 (2005), 15; Matthias Ruffert Perspektiven des Internationalen Verwaltungsrechts, in: Möllers/VoBkuhle/Walter (Hrsg.) (Fn. 6), 395 (,Internationales Verwaltungsrecht im Werden“); Breining-Kaufmann Internationales Verwaltungsrecht (Fn. 7), 72 („Rechtsgebiet sui generis“); vgl. auch bereits Thomas Fleiner-Gerster Grundzüge des allgemeinen und schweizerischen Verwaltungsrechts, 2. Aufl. 1980, § 10 Rn. 9 („In der Tat kann man bereits heute von einem eigenständigen internationalen Verwaltungsrecht sprechen.").

11 Vgl. z.B. Ohler Kollisionsordnung (Fn. 6) 2 ff. (mit weiteren Hinweisen); Matthias Ruffert Rechtsquellen und Rechtsschichten des Verwaltungsrechts, in: Wolfgang Hoffmann-Riem/Eberhard Schmidt-ABmann/Andreas Voßkuhle (Hrsg.), Grundlagen des Verwaltungsrechts (GVwR), Band I, 2006, § 17, Rz. 148 ff.; Werner Meng Extraterritoriale Jurisdiktion im öffentlichen Wirtschaftsrecht, 1994, $187 \mathrm{f}$.

12 Vgl. Donato Donati I trattati internazionali nel diritto costituzionale I, 1906, 430 ff., sowie die Hinweise bei Klaus Vogel Administrative Law, International Aspects, in: Rudolf Bernhardt (ed.), Encyclopedia of Public International Law, Volume I, 1992, 22 (23f.). 
sischen Sprache ${ }^{13}$, fehlt jedoch in der deutschen Sprache. Der Begriff "Internationales Verwaltungsrecht" bezeichnete hier lange Zeit vor allem das auf Sachverhalte mit Auslandsbezug gemünzte Verwaltungskollisionsrecht $t^{14}$ - in umstrittener begrifflicher Parallele zum (ebenfalls weitgehend national geregelten) „Internationalen Privatrecht“ (IPR). ${ }^{15}$ Noch im Jahre 1999 wurde konstatiert, dass dieses kollisionsrechtliche Begriffsverständnis das geläufige sei. ${ }^{16}$ Als „Internationales Verwaltungsrecht" wurde und wird daneben auch das „Binnenrecht" von Internationalen Organisationen (insb. das internationale Beamtenrecht) bezeichnet $^{17}$, weiter auch das in Gebieten, die unter UNO-Verwaltung stehen, zur Anwendung kommende „Recht der internationalen Verwaltung“.18 Darauf wird im Folgenden nicht näher eingegangen.

Neuerdings wird unter dem Titel „Internationales Verwaltungsrecht" (oder „Recht der internationalen Verwaltungsbeziehungen“"19) die ein-

13 Droit administratif international bzw. droit international administratif. Dazu Nguyen Droit administratif international (Fn. 7), 80; José Gascon y Marin Les transformations du droit international administratif, in: Recueil des Cours (RdC) 34 (1930 IV), 1 (21 ff.); vgl. auch ders. Tratado de Derecho administrativo II, 12. Aufl. 1952, 7 f.; (für die englische Rechtssprache) Vogel Administrative Law, International Aspects (Fn. 12), 23 (international administrative law bzw. administrative international law). - Zum konkurrierenden Begriff global administrative law vgl. Fn. 25.

${ }^{14}$ Grundlegend Karl Neumeyer Internationales Verwaltungsrecht, 4 Bände, 19101936. Vg1. auch Giovanni Biscottini, Diritto amministrativo internazionale, 2 Bände, 1964/66; Gerhard Hoffmann Internationales Verwaltungsrecht, in: Ingo von Münch (Hrsg.), Besonderes Verwaltungsrecht, 7. Aufl. 1985, $851 \mathrm{ff}$. (in späteren Auflagen nicht mehr enthalten); aus jüngerer Zeit Breining-Kaufmann Internationales Verwaltungsrecht (Fn. 7), $18 \mathrm{ff}$;; Christine Linke Europäisches Internationales Verwaltungsrecht, 2001; Meng Extraterritoriale Jurisdiktion (Fn. 11), $186 \mathrm{ff}$.; Nguyen Droit administratif international (Fn. 7), 85 ff.; Ohler Kollisionsordnung (Fn. 6). - Gegen ein spezielles Kollisionsrecht im öffentlichen Recht sprechen sich aus: Ulrich Häfelin/Georg Müller/Felix Uhlmann Allgemeines Verwaltungsrecht, 5. Aufl. 2006, Rn. 357.

is Zur Kontroverse vgl. Ohler Kollisionsordnung (Fn. 6), 3 (mit Hinweisen); aus der älteren Literatur Klaus Vogel Der räumliche Anwendungsbereich der Verwaltungsrechtsnorm, 1965, insb. $298 \mathrm{ff}$.; Franz Matscher Gibt es ein Internationales Verwaltungsrecht?, FS Günther Beitzke, 1979, 641.

${ }^{16}$ So Stefan Kadelbach Allgemeines Verwaltungsrecht unter europäischem Einfluss, 1999, 3 (mit Fn. 18). Vgl. auch Schmidt-Aßmann Herausforderung (Fn. 4), $335 \mathrm{f}$.

${ }_{17}$ Vgl. z.B. C.F. Amerasinghe International Administrative Law in the Twenty-First Century, FS Christopher Weeramantry 1998, 477 ff.; Battini Amministrazioni (Fn. 6), $47 \mathrm{ff}$.; Ruffert Rechtsquellen, in: Hoffmann-Riem/Schmidt-ABmann/Voßkuhle (Hrsg.), GVwR I (Fn. 11), § 17, Rz. 149 f.

${ }^{18} \mathrm{Vgl}$. Ruffert Rechtsquellen, in: Hoffmann-Riem/Schmidt-Aßmann/Voßkuhle (Hrsg.), GVwR I (Fn. 11), §17, Rz. 152; Verena Meyer Die internationale Verwaltung durch die Vereinten Nationen, 2006.

19 Begriff bei Schmidt-ABmann Herausforderung (Fn. 4), 317. 
gangs angesprochene Annäherung von Völkerrecht und Verwaltungsrecht erörtert. Man könnte dies als eine Rückkehr zu einem älteren, im 19. Jahrhundert wurzelnden Begriffsverständnis deuten. ${ }^{20}$ Man sollte jedoch nicht vergessen, dass sich der völkerrechtliche wie der verfassungsrechtliche Rahmen seither grundlegend gewandelt haben.

\section{b. Problemorientierter Ansatz}

Wie soll man den Begriff „Internationales Verwaltungsrecht" sinnvollerweise verstehen und einsetzen? Mir scheint es zweckmässig, vom zuletzt genannten neueren Begriffsverständnis auszugehen ${ }^{21}$, auch wenn es sich hierbei nicht um eine ideale juristische Begriffsbildung handelt, zumal sie mit der kollisionsrechtlichen Begriffstradition kollidiert. ${ }^{22}$ Doch für das Kollisionsrecht gibt es gute begriffliche Alternativen. ${ }^{23}$ Und der Begriff „Internationales Verwaltungsrecht" eignet sich besser als andere Begriffe, um die zunehmenden Wechselwirkungen zwischen Völkerrecht und Verwaltungsrecht kurz und prägnant zu bezeichnen. Dies verdeutlicht ein Blick auf einige potenzielle Alternativen. ${ }^{24}$

20 Zur Verwendung des Begriffs "Internationales Verwaltungsrecht" in der zweiten Hälfte des 19. Jahrhunderts, namentlich im Zusammenhang mit neu entstehenden „Verwaltungsunionen“ (wie der Internationalen Telegraphen-Union, 1865, heute Internationale Fernmeldeunion, oder dem Weltpostverein, 1875) vgl. Vec Recht und Normierung (Fn. 1), $147 \mathrm{ff}$ - Zur Rolle der Lehren Robert von Mohls und Lorenz von Steins vgl. Tietje Internationalisiertes Verwaltungshandeln (Fn. 1), 50 ff. Zur institutionellen und funktionalen "Verzahnung internationaler und nationaler Verwaltung“ vgl. auch Jost Delbrück Internationale und nationale Verwaltung - Inhaltliche und institutionelle Aspekte, in: Kurt G. A. Jeserich/Hans Pohl/Georg-Christoph von Unruh (Hrsg.), Deutsche Verwaltungsgeschichte, Band 5, 1987, 386 (396ff.).

${ }^{21}$ Für eine entsprechende Neudefinition des "Internationalen Verwaltungsrechts" Eberhard Schmidt-ABmann Verfassungsprinzipien für den europäischen Verwaltungsverbund, in: Hoffmann-Riem/Schmidt-Aßmann/Voßkuhle (Hrsg.), GVwR I (Fn. 11), 5 , Rn. 47.

22 Dies heisst nicht, dass das Verwaltungskollisionsrecht aus dem Begriff des "Internationalen Verwaltungsrechts" ausgeklammert werden müsste (Kollisionsrecht als Teil eines weit verstandenen „Internationalen Verwaltungsrechts ${ }^{4}$ ). Für eine Zusammenführung des „internationalisierten Verwaltungsrechts“ und des kollisionsrechtlichen Ansatzes Christoph Ohler Die Entwicklung eines Internationalen Verwaltungsrechts als Aufgabe der Rechtswissenschaft, DVB1 2007, 1083 (1087).

23 Vgl. Ohler Kollisionsordnung (Fn. 6), 3 („öffentliches Kollisionsrecht“ statt „Internationales Verwaltungsrecht").

$24 \mathrm{Vgl}$. auch Franz C. Mayer Internationalisierung des Verwaltungsrechts?, in: Möllers/Voßkuhle/Walter (Hrsg.) (Fn. 6), 54 ff. 
Der Begriff "globales Verwaltungsrecht" 25 - ein sehr weit gespannter Begriff - ist für unser Phänomen paradoxerweise zu eng, denn er grenzt unnötigerweise regionale und grenznachbarliche Aspekte aus ${ }^{26}$, die jedenfalls in Europa, insbesondere für die Schweiz, hoch bedeutsam sind.27 Der Begriff „transnationales Verwaltungsrecht ${ }^{28}$ orientiert sich $\mathrm{zu}$ sehr „zur Seite hin“ und vernachlässigt damit, tendenziell, die vertikale Komponente unseres Phänomens. Mit Begriffen wie „Völkerverwaltungsrecht" 29 oder „Verwaltungsvölkerrecht" rückt zu sehr eine bestimmte Rechtsschicht bzw. Rechtsquelle - das Völkerrecht - in den Vorder-

${ }^{25}$ Vgl. z. B. Sabino Cassese Il diritto amministrativo globale, Rivista trimestrale di diritto pubblico 2005, 331 (englische Fassung in: N.Y.U. Journal of International Law and Politics 37, 2005, 663); Lorenzo Casini Diritto amministrativo globale, in: Sabino Cassese (Hrsg.), Dizionario di diritto pubblico, 2006; Daniel C. Esty Good Governance at the Supranational Scale: Globalizing Administrative Law, Yale Law Journal 115 (2006), 1490; Kingsbury/Krisch/Stewart Emergence (Fn. 10), 15; Nico Krisch The Pluralism of Global Administrative Law, European Journal of International Law 17 (2006), 247; Ruffert Perspektiven, in: Möllers/Voßkuhle/Walter (Hrsg.) (Fn. 6), [403 ff.].

26 Ähnlich Schmidt-ABmann Herausforderung (Fn. 4), 317. - Bemerkenswert ist (aus schweizerisch-deutscher Sicht) der Vertrag vom 23. November 1964 über die Einbeziehung der Gemeinde Büsingen am Hochrhein in das schweizerische Zollgebiet (Systematische Rechtssammlung, SR, 0.631.112.136). In Art. 2 des Vertrags werden (in Form eines dynamischen Verweises) zahlreiche Gesetzgebungsbereiche aufgelistet, in denen - Sonderregelungen vorbehalten - in Büsingen „die schweizerischen (eidgenössischen und kantonalen) Rechts- und Verwaltungsvorschriften Anwendung ${ }^{\star}$ finden, unter anderem „d.) aus dem Bereich des Gesundheitswesens: [...] 6.) Verkehr mit Giften“, so dass in Büsingen auch das eidgenössische Giftgesetz vom 21. März 1969 (Kürzel: GG) zur Anwendung kam, bis dieses im Jahr 2005 durch das Chemikaliengesetz abgelöst wurde (vgl. Ziffer I des Anhangs zum Bundesgesetz vom 15. Dezember 2000 über den Schutz vor gefährlichen Stoffen und Zubereitungen, ChemG; SR 813.1).

27 Vgl. Daniel Thürer u.a. (Hrsg.) Bilaterale Verträge I und II Schweiz-EU. Handbuch, 2. Aufl. 2007. Dem Versuch, mittels sektorieller Abkommen Binnenmarktverhältnisse herzustellen, sind Grenzen gesetzt. Was aus deutscher oder österreichischer Sicht an der Schnittstelle zwischen Gemeinschaftsrecht (inkl. Verträge mit Drittstaaten) und nationalem Recht liegt (und als "Europäisierung“ des Verwaltungsrechts wahrgenommen wird), liegt aus schweizerischer Sicht an einer Schnittstelle zwischen Völkerrecht und Landesrecht („Internationalisierung“).

${ }^{28}$ Zum (uneinheitlichen) Begriffsgebrauch vgl. etwa Manfred Baldus Transnationales Polizeirecht, 2001, $47 \mathrm{ff}$; Mayer Internationalisierung des Verwaltungsrechts?, in: Möllers/VoBkuhle/Walter (Hrsg.) (Fn. 6), 54 ff. Vgl. auch Philip C. Jessup Transnational Law, 1956.

${ }^{29}$ So z. B. Christoph Möllers Transnationale Behördenkooperation - Verfassungs- und völkerrechtliche Probleme transnationaler administrativer Standardsetzung, ZaöRV 65 (2005), 351 (384); Breining-Kaufmann Internationales Verwaltungsrecht (Fn. 7), 68. Vgl. auch Alfred Verdross Völkerrecht, 5. Aufl. 1964, 590 („völkerrechtliches Verwaltungsrecht ${ }^{\text {)}}$ ). 
grund. Zwar geht es beim hier interessierenden Phänomen im Wesentlichen um „im Völkerrecht gegründete(s) Verwaltungsrecht““.30 Man sollte jedoch die starke nationale Komponente nicht vorschnell ausblenden. Einzubeziehen sind auch informelle Abstimmungsprozesse, die ohne förmliche völkerrechtliche Bindungen auskommen (Stichwort: Empfehlungen internationaler Organisationen oder Gremien ${ }^{31}$ ). Zu dieser Kategorie der informellen Abstimmungsprozesse gehört auch der in der Schweiz verbreitet praktizierte sog. „autonome Nachvollzug“ von Gemeinschaftsrecht. ${ }^{32}$ Dieser „Nachvollzug“ wird oft belächelt, und er ist nicht frei von Skurrilitäten, wie ein Blick in die schweizerische Lebensmittelgesetzgebung offenbart mit ihren am Recht der EG geschulten Legaldefinitionen für derart identitätsstiftende Produkte wie Emmentaler Käse oder Milchschokolade. ${ }^{33}$ Näher betrachtet (besonders aus der Sicht der Exportwirtschaft) ist der „autonome Nachvollzug“ eine durchaus rationale Angelegenheit und in der heute mehrheitlich integrationskritischen Schweiz bezeichnenderweise politisch nur wenig umstritten. ${ }^{34}$

${ }^{30}$ Schmidt-ABmann Verfassungsprinzipien, in: Hoffmann-Riem/Schmidt-Aßmann/ VoBkuhle (Hrsg.), GVwR I (Fn. 11), §5, Rn. 48.

${ }^{31}$ Ein oft zitiertes Beispiel sind die Empfehlungen des sog. Basler Ausschusses für Bankenaufsicht (Basel Committee on Banking Supervision). Vgl. Anne van Aaken Transnationales Kooperationsrecht nationaler Aufsichtsbehörden als Antwort auf die Herausforderung globalisierter Finanzmärkte, in: Möllers/Voßkuhle/Walter (Fn. 6), 219 (227ff.); Michael S. Barr/Geoffrey P. Miller Global Administrative Law: The View from Basel, European Journal of International Law 17 (2006), 15.

32 Vgl. Tobias Jaag Europarecht - Die europäischen Institutionen aus schweizerischer Sicht, 2003, $395 \mathrm{ff}$. Zur „Europaverträglichkeit" als Gesetzgebungsmaxime vgl. Martin Philipp Wyss Europakompatibiliät und Gesetzgebungsverfahren im Bund, Aktuelle Juristische Praxis 2007, 717.

$33 \mathrm{Vgl}$. Art. 39 Abs. 3 sowie Anhang 4 der Verordnung des Eidgenössischen Departements des Innern (EDI) vom 23. November 2005 über Lebensmittel tierischer Herkunft (SR 817.022.108) sowie Art. 36 der Verordnung des EDI vom 23. November 2005 über Zuckerarten, süsse Lebensmittel und Kakaoerzeugnisse (SR 817.022.101). Kein direktes Vorbild im Gemeinschaftsrecht scheint die Legaldefinition in Art. 40 (Satz 1) derselben Verordnung zu haben: „Milchschokoladestreusel oder Milchschokoladeflocken sind Milchschokoladen in Form von Streuseln oder Flocken."

${ }^{34}$ Ein gewisser Widerstand erwächst jetzt allerdings in der Schweiz dem Vorhaben, im Verhältnis zu den Staaten des Europäischen Wirtschaftsraums (EU, EFTA) einseitig das Herkunftsortsprinzip (Cassis-de-Dijon-Prinzip) einzuführen (vgl. Vorentwurf vom 29. November 2006 für eine Teilrevision des Bundesgesetzes vom 6. Oktober 1995 über die technischen Handelshemmnisse, THG; SR 946.51). 
Der Begriff „Internationalisierung des Verwaltungsrechts“35 schliesslich - gebildet in Analogie zur „Europäisierung“ des Verwaltungsrechts ${ }^{36}$ - ist $\mathrm{zu}$ defensiv und zu einseitig angelegt. Das hier interessierende Phänomen umfasst neben Einwirkungen des internationalen Rechts auf das nationale Recht - von „oben nach unten“ („Internationalisierung") - auch bedeutsame (und nicht weniger interessante) Einwirkungen in der Gegenrichtung - von „unten nach oben“. Man könnte von einer "Ver-Verwaltungsrechtlichung des internationalen Rechts" sprechen (wenn dies nicht so ein „Zungenbrecher" wäre). ${ }^{37}$

\section{Grundprobleme eines „Internationalen Verwaltungsrechts“}

\section{Suche nach einem Ordnungsrahmen}

Mit diesen begrifflichen Klärungen hat zugleich der Untersuchungsgegenstand etwas klarere Konturen gewonnen, ohne dass allerdings das Phänomen selbst schon voll erfasst oder durchdrungen wäre. Was soll das „Internationale Verwaltungsrecht“ leisten? Welche Leistungen kann und soll die Rechtswissenschaft bei der „Entwicklung eines Internationalen Verwaltungsrechts" erbringen? In der Literatur geht man auf unterschiedliche Weise an das Phänomen heran. Ohne Anspruch auf Vollständigkeit seien hier einige Ansätze genannt.

Verbreitet sind Fallstudien zu einzelnen - besonders prädestinierten Rechtsgebieten, etwa zum Finanzmarktaufsichtsrecht, zum Sozial(verwaltungs)recht, zum Umweltrecht, zum Gesundheits(verwaltungs)recht oder zum technischen Sicherheitsrecht. ${ }^{38}$ So wichtig derartige Fallstu-

${ }^{35} \mathrm{Vgl}$. z.B. Eberhard Schmidt-Aßmann Internationalisierung des Verwaltungsrechts, FS Reiner Schmidt, 2006, 149 ff.; Mayer Internationalisierung (Fn. 6); Karl-Heinz Ladeur Die Internationalisierung des Verwaltungsrechts, in: Möllers/Voßkuhle/Walter (Hrsg.) (Fn. 6), $375 \mathrm{ff}$.

$36 \mathrm{Vgl}$. (statt vieler) Eberhard Schmidt-Aßmann Das Allgemeine Verwaltungsrecht als Ordnungsidee, 2. Aufl. 2004, 377 ff.; Jürgen Schwarze Europäisches Verwaltungsrecht, 2. Aufl. 2005; Manfred Zuleeg/Hans-Werner Rengeling Deutsches und europäisches Verwaltungsrecht, VVDStRL 53 (1994), 154 bzw. 202.

37 In Parallele zur „Konstitutionalisierung des Völkerrechts“ - verstanden als Durchdringung des internationalen Rechts mit Elementen der Verfassungsidee - kann man die „Ver-Verwaltungsrechtlichung des internationalen Rechts“ auffassen als ein Bemühen um Durchdringung des Völkerrechts mit der "Idee des Verwaltungsrechts".

38 Vgl. Möllers/Voßkuhle/Walter (Hrsg.) (Fn. 6) mit Beiträgen zum Entwicklungsverwaltungsrecht (Philipp Dann, Franz C. Mayer), zum Internationalen Sozialverwaltungsrecht (Markus Glaser), zum Internationalen Umweltverwaltungsrecht (Wolfgang Durner, 
dien sind, so klar ist doch zugleich - auch den jeweiligen Autoren -, dass es eines sachgebietsübergreifenden Bezugs- oder Ordnungsrahmens bedarf, um aus der Fülle des Materials gebietsübergreifende Erkenntnisse gewinnen zu können. ${ }^{39}$ Andere Untersuchungen stellen Querschnittsphänomene wie die Verwaltungskooperation oder die Bildung von (Behörden-) Netzwerken ins Zentrum. ${ }^{40}$ Wieder andere befassen sich mit Rechtsschutz- oder Legitimationsfragen ${ }^{41}$ oder mit Einzelphänomenen wie etwa dem transnationalen Verwaltungsakt ${ }^{42}$. Eine beträchtliche Resonanz hat die (adressaten- bzw. tätigkeitsbezogene) Untergliederung des „Internationalen Verwaltungsrechts“ in die drei Funktionskreise „Aktionsrecht" (für Verwaltungsinstanzen auf internationaler Ebene), "Determinationsrecht" (für die nationalen Verwaltungsrechtsordnungen) und „Kooperationsrecht" erlangt (Schmidt-A $\beta$ mann).$^{43}$ Wieder andere Untersuchungen widmen sich der Frage nach

Matthias Rossi), zum Transnationalen Steuerrecht (Ekkehart Reimer, Christian Tietje), zum Finanzmarktaufsichtsrecht (Anne van Aaken, Christoph Ohler), zum Migrationsverwaltungsrecht (Jürgen Bast, Nico Krisch), zur Internationalen Standardsetzung (Hans Christian Röhl, Oliver Lepsius); Kingsbury/Krisch/Stewart/Wiener (Fn. 6); Krisch/Kingsbury (Fn. 6). Mayer Internationalisierung (Fn. 6) behandelt das Ausländerrecht und das Internetverwaltungsrecht, Tietje Internationalisiertes Verwaltungshandeln (Fn. 1) das Gesundheitsverwaltungsrecht, das Umweltverwaltungsrecht sowie das Kommunikations- und Transportverwaltungsrecht. - Zur Unterschiedlichkeit der Erfahrungen mit dem Internationalisierungs-Phänomen in verschiedenen Rechtsgebieten Ladeur Internationalisierung, in: Möllers/Voßkuhle/Walter (Hrsg.) (Fn. 6), $379 \mathrm{ff}$.

39 Vgl. Möllers Internationales Verwaltungsrecht, in: ders./Voßkuhle/Walter (Hrsg.) (Fn. 6), 4; Gernot Sydow Verwaltungskooperation in der Europäischen Union, 2004, $120 \mathrm{ff}$.

${ }^{40}$ Vgl. z.B. Anne-Marie Slaughter A New World Order, 2004. - Zu Phänomen und Begriff vgl. (kritisch) Möllers Behördenkooperation (Fn. 29), 380ff.; Sydow Verwaltungskooperation (Fn. 39), $78 \mathrm{ff}$. Vgl. auch Sigrid von Boysen u.a. (Hrsg.) Netzwerke, 2007; Gunnar Folke Schuppert Verwaltungsorganisation als Steuerungsfaktor, in: Hoffmann-Riem/Schmidt-Aßmann/Voßkuhle (Hrsg.), GVwR I (Fn. 11), § 16, Rn. $167 \mathrm{ff}$. („Von Internationalisierung zu Transnationalisierung“).

${ }^{41}$ Vgl. Stefan Burbaum Rechtsschutz gegen transnationales Verwaltungshandeln, 2003; Oliver Lepsius Standardsetzung und Legitimation, in: Möllers/Voßkuhle/Walter (Hrsg.) (Fn. 6), 345; Ruffert Perspektiven, in: Möllers/Voßkuhle/Walter (Hrsg.) (Fn. 6), 395 (406ff.).

42 Vgl. Volker Nessler Der transnationale Verwaltungsakt, NVwZ 1995, 864; Bernhard Raschauer Transnationale Verwaltungsakte, FS Theo Öhlinger, 2004, 661; Matthias Ruffert Der transnationale Verwaltungsakt, Die Verwaltung 34 (2001), $453 \mathrm{ff}$.

${ }^{43}$ Schmidt-Aßmann Herausforderung (Fn. 4), $336 \mathrm{ff}$. Vgl. auch die Beiträge von Dann, Glaser, Reimer, Tietje und Ruffert in: Möllers/Voßkuhle/Walter (Fn. 6), 41, 77, 189f., 210 ff. bzw. 405. 
den Möglichkeiten eines Transfers von Rechtsfiguren des nationalen Verwaltungsrechts auf die völkerrechtliche Ebene. ${ }^{44}$

Diesen und weiteren Ansätzen verdanken wir sehr wertvolle Beiträge zum besseren Verständnis des hier interessierenden Phänomens. Allerdings fällt es nach wie vor nicht leicht, die dabei gewonnenen Erkenntnisse einzuordnen. Einprägsame Bilder und Schlagworte - wie „globaler Verwaltungsraum “45 oder (Behörden-),,Netzwerk ${ }^{\text {“46 } ~-~ k o ̈ n n e n, ~ b e i ~ a l l e n ~}$ Vorbehalten, Wichtiges leisten, indem sie die Aufmerksamkeit auf bisher zu wenig beachtete Aspekte und Zusammenhänge lenken. Der Beitrag zur Problemdurchdringung bleibt indes gering. ${ }^{47}$

Was bei der „Entwicklung eines Internationalen Verwaltungsrechts“ vorangetrieben werden sollte, ist heute weniger die Suche nach weiteren Einzelphänomenen, Schlagworten oder Referenzgebieten als vielmehr die Suche nach einem geeigneten Bezugs- und Ordnungsrahmen. ${ }^{48}$

Wie und wo könnte man einen solchen Bezugsrahmen finden? Einen Weg weist die (an das Tagungsmotto anknüpfende) Frage, worin denn die Leistung besteht, die man vom Verwaltungsrecht und "seiner" Wissenschaft erwarten darf. Die zentrale Aufgabe des Verwaltungsrechts im modernen Verfassungsstaat ist die Gewährleistung eines rechtsstaatlich einwandfreien, demokratisch abgestützten Handelns der staatlichen Verwaltung. ${ }^{49}$ Das Aufgabenspektrum reicht aber darüber hinaus. Dies offenbart etwa ein Blick auf die Kriterien der Verwaltungskontrolle, wie sie

44 Vgl. z. B. Esty Globalizing Administrative Law (Fn. 25), 1523 ff. (wo eine „basic global administrative law toolbox“ präsentiert wird); Richard B. Stewart U.S. Administrative Law: A Model for Global Administrative Law?, Law and Contemporary Problems 68 (2005), 63 (76ff.); Carol Harlow Global Administrative Law: The Quest for Principles and Values, European Journal of International Law 17 (2006), 187 (198ff.). Es zeigt sich hier eine gewisse Parallele zur „Konstitutionalisierung“ des Völkerrechts. Vgl. vorne Fn. 37.

45 Vgl. Kingsbury/Krisch/Stewart Emergence (Fn. 10), 15 (26): „global administrative space“. - Zum schwer fassbaren Begriff ,global governance“ vgl. Ruffert Globalisierung (Fn. 3), $24 \mathrm{ff}$.

46 Vgl. vorne Fn. 40.

47 Dies gilt auch für das Schlagwort der „Entterritorialisierung“ des Verwaltens bzw. des Verwaltungsrechts (vgl. z.B. Ladeur Internationalisierung, in: Möllers/VoBkuhle/ Walter (Hrsg.) (Fn. 6), 376, 393). Auch der inzwischen geläufige Begriff „Verwaltungsverbund“ bleibt in seiner Leistungsfähigkeit begrenzt.

$48 \mathrm{Vgl}$. Möllers Internationales Verwaltungsrecht, in: ders./Voßkuhle/Walter (Hrsg.) (Fn. 6), $4 \mathrm{f}$.

${ }^{49} \mathrm{Zu}$ den rechtsstaatlich-demokratischen Grundfunktionen des Verwaltungsrechts vgl. (statt vieler) Eberhard Schmidt-Aßmann Das allgemeine Verwaltungsrecht als Ordnungsidee, 2. Aufl. 2004, 43 ff.; Pierre Moor Droit administratif, Vol. I: Les fondements généraux, 2. Aufl. 1994, chiffres 3 ff. 
in der schweizerischen Gesetzgebung im Jahr 1990 kodifiziert (und davor schon praktiziert) wurden. Die staatliche Verwaltung soll rechtmässig, zweckmässig, gleichmässig, wirksam und wirtschaftlich handeln. ${ }^{50}$ Diese Kriterien stehen natürlich nicht unverbunden nebeneinander, und sie überschneiden sich mit den rechtsstaatlich-demokratischen Anforderungen. Es ist Aufgabe des Verwaltungsrechts, den Prozess der verwaltungsmässigen Rechtsverwirklichung so zu organisieren - d.h. Strukturen, Instrumente und Abläufe so zu gestalten -, dass die (demokratisch verantworteten) Regelungen vorgabengetreu vollzogen werden. Verwaltungsrecht ist, so gesehen, in weiten Teilen Rechtsverwirklichungsrecht. Dies gilt nicht nur für das Allgemeine Verwaltungsrecht, sondern auch für viele Regelungen des Besonderen Verwaltungsrechts. ${ }^{51}$

Für die „Entwicklung eines Internationalen Verwaltungsrechts“ empfiehlt es sich, an die Grundmuster und Grundanforderungen des Rechtsverwirklichungsprozesses anzuknüpfen. Worum geht es? Es geht darum, Regeln zu bilden, Regeln verwaltungsmässig umzusetzen und anzuwenden rechtmässig, zweckmässig, gleichmässig usw. (wobei „gleichmässig“ nicht bedeutet: zwingend immer und überall genau gleich, sondern: innerhalb jener Bandbreite, auf die man sich bei der Festlegung der Vorgaben, national oder international, verständigt hat).

\section{Strukturen der Rechtsverwirklichung im Bereich des Verwaltungsrechts}

Die administrative Verwirklichung rechtlicher Regelungen ist eine anspruchsvolle Aufgabe. Schon im denkbar einfach gelagerten Fall der kleinen, überschaubaren, gut durchorganisierten Verwaltung - mit klaren Weisungshierarchien und kurzen Wegen zwischen Verwaltungsspitze und Vollzugspersonal - ergeben sich Schwierigkeiten unter-

so In Anlehnung an die Kriterien parlamentarischer Verwaltungskontrolle gemäss Art. 26 Abs. 3 des Bundesgesetzes vom 13. Dezember 2002 über die Bundesversammlung (Parlamentsgesetz, ParlG; SR 171.10): „Die Bundesversammlung übt die Oberaufsicht nach den folgenden Kriterien aus: a. Rechtmässigkeit; b. Ordnungsmässigkeit; c. Zweckmässigkeit; d. Wirksamkeit; e. Wirtschaftlichkeit.“ Vgl. auch Philippe Mastronardi Kriterien der demokratischen Verwaltungskontrolle, 1991.

s1 Zum Verhältnis von Allgemeinem Verwaltungsrecht und Fachverwaltungsrecht vgl. Martin Burgi Rechtsregime, in: Hoffmann-Riem/Schmidt-ABmann/Voßkuhle (Hrsg.), GVwR I (Fn. 11), § 18, Rn. 96ff. (mit weiteren Hinweisen). - In Titeln von Lehr- oder Handbüchern französischer oder italienischer Sprache ist das Adjektiv ,allgemein" eher unüblich (vgl. immerhin René Chapus Droit administratif général, tome 1, 15. Aufl. 2001; Sabino Cassese Trattato di diritto amministrativo. Diritto amministrativo generale, 2 Bände, 2. Aufl. 2003); die Unterscheidung besteht der Sache nach jedoch auch hier. 
schiedlichster Art. ${ }^{52}$ Es genügt daher nicht, materielle Regeln vorzugeben. Um eine ordnungsgemässe Aufgabenerfüllung im Sinne der demokratisch verantworteten Vorgaben sicherzustellen, ist ein ganzes Arsenal an Instrumenten und Sicherungen erforderlich. Stichworte dazu: allgemeine und einzelfallbezogene Weisungen, organisatorischverfahrensmässige Vorkehrungen, interne und externe Kontrollen usw.

Die Schwierigkeiten nehmen erheblich zu, wenn die Rechtsverwirklichung gemeinwesenübergreifend organisiert ist - wie dies beim Verwaltungsvollzug des Bundesrechts in Deutschland, in Österreich und in der Schweiz in unterschiedlichen Variationen der Fall ist. ${ }^{53}$ Das Ziel ist stets dasselbe: ein rechtmässiger, gleichmässiger, wirksamer usw. Vollzug. In diesem „vollzugsföderalistischen" Modell der Verwaltungsrechtsverwirklichung sind die Einwirkungsmöglichkeiten des Bundes jedoch begrenzt, weil - aus föderalistischen Rücksichten - die herkömmlichen Instrumente und Mechanismen der Verwaltungsaufsicht hier nicht oder nur in abgeschwächter Form zur Verfügung stehen ${ }^{54}$ (Bundesaufsicht statt Verwaltungsaufsicht).

Noch komplexer ist die Lage beim Vollzug des Gemeinschaftsrechts durch die Verwaltungen der EU-Mitgliedstaaten. ${ }^{55}$ Es bestehen 2 war auch hier gewisse Aufsichtsmittel wie etwa die Inspektion ${ }^{56}$, und es ist nicht ausgeschlossen, dass man selbst in Drittstaaten europäischen In-

52 Dieses an einheitsstaatlichen Strukturen orientierte Modell spielte und spielt für die Entwicklung der Allgemeinen Lehren des Verwaltungsrechts eine zentrale Rolle. Zu den Folgen gehört eine gewisse „Föderalismusblindheit“ des Verwaltungsrechts. Vgl. Giovanni Biaggini Theorie und Praxis des Verwaltungsrechts im Bundesstaat, 1996, $263 \mathrm{ff}$., $308 \mathrm{ff}$. - Zur Bedeutung des Hierarchieprinzips vgl. Horst Dreier Hierarchische Verwaltung im demokratischen Staat, 1991; Pierre Moor Droit administratif, Vol. III, 1992, chiffre 1.2.1.

53 Vgl. (statt vieler) Peter Badura Staatsrecht, 3. Aufl. 2003, 609 ff.; Ulrich Häfelin/Walter Haller Schweizerisches Bundesstaatsrecht, 6. Aufl. 2006, 315f,, 325ff.; Peter Pernthaler Österreichisches Bundesstaatsrecht, 2004, $371 \mathrm{ff}$. - Weitere Fälle des gemeinwesenübergreifenden Verwaltungsvollzugs sind die verwaltungsmässige Verwirklichung (glied)staatlichen Rechts durch Gemeinden oder der Vollzug von rechtsetzenden Verträgen zwischen Gliedstaaten durch die Verwaltungen der beteiligten Gliedstaaten (in der Schweiz: interkantonale Verträge; vgl. Art. 48 BV.

54 Dazu (mit rechtsvergleichenden Hinweisen) Thomas Groß Verantwortung und Effizienz in der Mehrebenenverwaltung, VVDStRL 66 (2007), 152 (163 ff.); Biaggini Theorie und Praxis (Fn. 52), $143 \mathrm{ff} ., 172 \mathrm{ff}$.

55 Zum dezentralen Vollzug und zu strukturellen Veränderungen in jüngerer Zeit vgl. Schwarze Europäisches Verwaltungsrecht (Fn. 36), CI ff., 33 ff.; Sydow Verwaltungskooperation (Fn. 39), $70 \mathrm{ff} ., 126 \mathrm{ff}$.

56 Zum rechtlichen Rahmen vgl. Antje David Inspektionen im Europäischen Verwaltungsrecht, 2003, $150 \mathrm{ff}$. 
spektoren begegnet (etwa auf Kontrollbesuch in der Schweiz). ${ }^{57}$ Das traditionelle verwaltungsrechtliche Arsenal ist hier aber nur sehr bedingt verfügbar. Dafür trifft man auf verschiedene europarechtliche „Ersatzstrategien“, etwa in Gestalt von Vorgaben an das mitgliedstaatliche Verwaltungsverfahrensrecht oder in Gestalt finanzieller Anreiz- oder Sanktionsmechanismen. ${ }^{58}$

Besonders komplex sind die Verhältnisse bei der verwaltungsmässigen Umsetzung von völkerrechtlichen oder aus Völkerrecht abgeleiteten Vorgaben. Auch hier sind die Ziele prinzipiell dieselben ${ }^{59}$ (immer vorausgesetzt, dass man es beim Festsetzen gemeinsamer völkerrechtlicher Vorgaben ernst gemeint hat ${ }^{60}$ ). Es besteht hier nicht selten sogar ein besonders ausgeprägtes Bedürfnis nach gleichgerichteter Auslegung und Handhabung der gemeinsamen Regeln, dies vor allem in wirtschaftsnahen Rechtsgebieten ${ }^{61}$, wo es oft auch um Fragen des internationalen (Standort-) Wettbewerbs geht. Die Festlegung von Mindeststandards und die Angleichung von Regeln verfehlen ihren Zweck, wenn Auslegung und Anwendung dieser Normen national divergieren. 62

Im völkerrechtlichen Verhältnis erfolgt die verwaltungsmässige Rechtsverwirklichung unter besonders erschwerten Bedingungen. Eine Aufsicht im herkömmlichen Sinn fehlt. Die Distanz zwischen Regelungsebene (international) und Vollzugsebene (national, lokal) ist hier besonders gross, das rechtliche Band ist sehr lose. Eine der Kernfragen

57 Vgl. etwa Anhang 7 (Handel mit Weinbauerzeugnissen) des Abkommens vom 21. Juni 1999 zwischen der Schweizerischen Eidgenossenschaft und der Europäischen Gemeinschaft über den Handel mit landwirtschaftlichen Erzeugnissen (SR 0.916.026.81).

$58 \mathrm{Vgl}$. Schwarze Europäisches Verwaltungsrecht (Fn. 36), CII ff.; Eberhard Schmidt$A \beta$ mann Der Europäische Verwaltungsverbund und die Rolle des Europäischen Verwaltungsrechts, in: ders./Bettina Schöndorf-Haubold (Hrsg.) Der europäische Verwaltungsverbund, 2005, 1 (20ff.). - Der Kohärenzsicherung im Binnenmarkt dienen auch Massnahmen auf dem Gebiet der Verwaltungsorganisation bzw. -kooperation. Vgl. z. B. Hans-Heinrich Trute Der europäische Regulierungsverbund in der Telekommunikation ein neues Modell europäisierter Verwaltung, FS Peter Selmer, 2004, 565 (570ff.).

s9 Vgl. auch Kingsbury/Krisch/Stewart Emergence (Fn. 10), 15 (16).

60 In der Praxis zeigt sich leider, dass dies nicht immer für alle Beteiligten zutrifft. Vgl. Helen Keller/Daniela Thurnherr Taking International Law Seriously, 2005.

61 Von grosser praktischer Bedeutung ist die einheitliche Handhabung von Mindeststandards etwa im Finanzmarktrecht, im technischen Sicherheitsrecht oder im Lebensmittelrecht. - Einen interessanten Ansatz zur Vermeidung von Auslegungsdivergenzen verkörpert der im Schoss der OECD entstandene Experten-Kommentar zum OECD-Musterabkommen zur Vermeidung der Doppelbesteuerung. Vgl. Ekkehart Reimer Transnationales Steuerrecht, in: Möllers/Voßkuhle/Walter (Hrsg.) (Fn. 6), 181 (187).

$62 \mathrm{Vgl}$. Schmidt-Aßmann Herausforderung (Fn. 4), 319. 
eines „Internationalen Verwaltungsrechts“ lautet daher: Wie kann man sicherstellen, dass Regelungen, auf die man sich international verständigt hat, gemäss den Intentionen der Urheber - und unter Wahrung rechtsstaatlich-demokratischer Standards - verwirklicht werden?

\section{Folgerungen und Folgefragen}

In einer kurzen Zwischenbilanz kann festgehalten werden: Die drei ebenenübergreifenden Vollzugskonstellationen (bundesstaatlich, gemeinschaftsrechtlich, völkerrechtlich) unterscheiden sich stark in Bezug auf die rechtlichen Rahmenbedingungen. Die verfolgten Ziele aber sind grundsätzlich dieselben (rechtmässiger, gleichmässiger Vollzug), und die zu lösenden Probleme sind strukturell ähnlich. Dies legt es nahe, bei der „Entwicklung eines Internationalen Verwaltungsrechts“ bundesstaatliche und gemeinschaftsrechtliche Erfahrungen fruchtbar zu machen. ${ }^{63}$ Aus der Fülle möglicher Folgefragen sollen hier einige besonders wichtige herausgegriffen werden (III.), bevor in einer Schlussbetrachtung (IV.) die Aufgaben der Rechtswissenschaft und die Einordnung und Beschaffenheit des "Internationalen Verwaltungsrechts“ zur Sprache kommen.

\section{Grundfragen eines „Internationalen Verwaltungsrechts“}

\section{Zentrale und dezentrale Rechtsverwirklichung als Grundmodelle}

Für die verwaltungsmässige Verwirklichung gemeinsamer Regelungen (ob vereinbart, ob gesetzt ${ }^{64}$ ) kommen zwei Grundmodelle in Betracht (je mit Variationsmöglichkeiten), nämlich der zentrale Vollzug durch eine Instanz auf völkerrechtlicher Ebene oder aber der dezentrale Vollzug durch eine Instanz der nationalen Ebene (allenfalls der lokalen oder gliedstaatlichen Ebene). ${ }^{65}$

${ }^{63}$ In diesem Sinne auch (mit Blick auf die Verwirklichung des Gemeinschaftsrechts) Groß Mehrebenenverwaltung (Fn. 54), 155; Hans Christian Röhl Akkreditierung und Zertifizierung im Produktesicherheitsrecht, 2000, 45.

64 Zur Rechtsetzung internationaler Organisationen vgl. z. B. José E. Alvarez International Organizations as Law-makers, 2005, 109 ff.; Jurij Daniel Aston Sekundärgesetzgebung internationaler Organisationen zwischen mitgliedstaatlicher Souveränität und Gemeinschaftsdisziplin, 2005.

65 Bereits Otto Mayer (Deutsches Verwaltungsrecht, Band II, 1. Aufl. 1895, 472 ff.) hat darauf aufmerksam gemacht, dass der Übergang zwischen direktem und delegiertem Vollzug fliessend ist. Zu Zwischenformen im Recht der Europäischen Union illustrativ Sydow Verwaltungskooperation (Fn. 39), 126ff. - Zum (umstrittenen) Mischverwal- 
Im ersten Grundmodell (zentraler Vollzug) entwickelt sich das Verwaltungsrecht internationaler Organisationen, eine der einleitend erwähnten Kategorien des „Internationalen Verwaltungsrechts“. Dieses Modell wird uns nicht weiter beschäftigen. Hier interessiert das unter dem Aspekt der Rechtsverwirklichung anspruchsvollere zweite Grundmodell - man könnte von „,ölkerrechtlichem Vollzugsföderalismus“ sprechen. Es handelt sich um eine besonders gelagerte Variante der Mehrebenenkonstellation, einem Thema der letztjährigen Tagung in Rostock. In den Rostocker Referaten über „Verantwortung und Effizienz in der Mehrebenenverwaltung" standen - im besagten Kontext mit gutem Grund - die Gemeinsamkeiten verschiedener Mehrebenenkonstellationen im Vordergrund. ${ }^{66}$

Mit Blick auf die „Entwicklung eines Internationalen Verwaltungsrechts" - und mit Blick speziell auf die zentrale Frage, wie man bundesstaatliche bzw. gemeinschaftsrechtliche Erfahrungen fruchtbar machen kann - tritt die Frage nach den Unterschieden in den Vordergrund. Näher betrachtet interessieren hier zwei Fragestellungen. Zum einen: Inwieweit sind der bundesstaatliche bzw. gemeinschaftsrechtliche Vollzugsföderalismus einerseits, die völkerrechtliche Konstellation andererseits vergleichbar, inwieweit unterscheiden sie sich? Zum zweiten: Wie geht man im bundesstaatlichen bzw. gemeinschaftsrechtlichen Vollzugsföderalismus vor, um das im Vergleich zum Modell des ,zentralen Verwaltungsvollzugs" bestehende strukturelle Defizit auszugleichen (Stichwort: Fehlen einer eigentlichen Verwaltungsaufsicht).

Zunächst zum zweiten Punkt: Das Bundesstaatsrecht hat, unterstützt durch die Rechtslehre, mit einigem Erfindungsgeist verschiedene Methoden und Instrumente entwickelt, die das strukturelle Defizit auszugleichen helfen. Um nur einige Ersatzstrategien zu nennen: Vorgaben an das gliedstaatliche Organisations- und Verfahrensrecht; Weisungen im Verhältnis Bund-Gliedstaaten ${ }^{67}$; Instrumentalisierung des Rechtsschutzsystems für Zwecke der Bundesrechtsdurchsetzung, dies etwa durch Aktivierung der Bürgerinnen und Bürger ${ }^{68}$ (die gewissermassen zu „Advokaten“ des Bundesrechts werden) oder auch - in der Schweiz

tungs-Verbot im deutschen Bundesstaatsrecht vgl. (statt vieler) Gross Mehrebenenverwaltung (Fn. 54), 162 (mit Hinweisen).

${ }^{66}$ Eckhard Pache/Thomas Groß Verantwortung und Effizienz in der Mehrebenenverwaltung, VVDStRL 66 (2007), 106 bzw. 152 (insb. 163 ff.). - Zum „Mehrebenen“-Begriff vgl. auch Christoph Möllers Gewaltengliederung, 2005, $210 \mathrm{ff}$.

${ }^{67}$ Siehe (mit rechtsvergleichenden Hinweisen) Gross Mehrebenenverwaltung (Fn. 54), 165, 168.

68 Vgl. Johannes Masing Die Mobilisierung des Bürgers für die Durchsetzung des Rechts, 1997. 
besonders ausgeprägt - durch Verleihung von besonderen Beschwerderechten an bestimmte private Verbände oder nationale Aufsichtsbehörden. ${ }^{69}$ Auf vergleichbare Strategien trifft man im Gemeinschaftsrecht. ${ }^{70}$

Diese Ansätze eignen sich als Ausgangsbasis für die Lösung der strukturell ähnlich gelagerten Probleme im völkerrechtlichen Kontext. Es wäre unklug, wenn man nicht versuchen würde, diesen reichen Erfahrungsschatz für die „Entwicklung eines Internationalen Verwaltungsrechts" zu nutzen. Vorschnelle Analogien allerdings führen nicht zum Ziel. $^{71}$ Vielmehr ist eine sorgfältige Analyse der Gemeinsamkeiten und der Unterschiede erforderlich.

\section{Gemeinsamkeiten und Unterschiede}

Vergleicht man die drei hier interessierenden Konstellationen - bundesstaatlich, gemeinschaftsrechtlich, völkerrechtlich - miteinander, so zeigen sich mehrere Gemeinsamkeiten. Beim rechtsebenenübergreifenden Verwaltungsvollzug ist das hierarchische Gefüge mehr oder weniger aufgebrochen. Angesichts der „Distanz“ zwischen rechtsetzenden und vollziehenden Instanzen ist mit erheblichen Spannungen und Interessengegensätzen zu rechnen, welche die Zielerreichung - Rechtmässigkeit, Gleichmässigkeit, Wirksamkeit des Verwaltungsvollzugs - gefährden können.72 Es besteht Bedarf nach besonderen Kontroll- bzw. Ausgleichsmechanismen. Hinzu kommt, dass im dezentralen Modell die mit dem Vollzug betrauten Stellen meist nicht nur Recht der oberen Ebene (d.h. des Bundesstaates, der EU oder der völkerrechtlichen Ebene) umzusetzen haben, sondern daneben auch für den Vollzug ,heimischen" (d.h. gliedstaatlichen, mitgliedstaatlichen oder staatlichen) Rechts zuständig sind. In Anlehnung an den französischen Völkerrechtler Georges Scelle kann man von einem „dédoublement fonctionnel“73

${ }^{69}$ Vgl. Art. 89 des Bundesgesetzes über das Bundesgericht (Bundesgerichtsgesetz, BGG; SR 173.110). Zur Instrumentalisierung des Rechtsschutzsystems für Zwecke der Bundesaufsicht vgl. Biaggini Theorie und Praxis (Fn. 52), $207 \mathrm{ff}$.

${ }^{70}$ Zum Instrumentarium der "vertikalen Koordination“ Gross Mehrebenenverwaltung (Fn. 54), 163 ff. (mit weiteren Hinweisen).

71 Vgl. Kingsbury/Krisch/Stewart Emergence (Fn. 10), 28.

72 Diese Gegensätze drohen aus dem Blickfeld zu geraten, wenn man die Rechtsverwirklichung primär unter dem Blickwinkel des „Kooperationsbedürfnisses“ analysiert (vgl. z.B. Pache Mehrebenenverwaltung (Fn. 66), 116) oder die ganz unterschiedlichen Zwecke der „Verwaltungskooperation“ (bei der es sich nicht um eine einheitliche, festgefügte Rechtsfigur handelt) ausblendet.

${ }^{73}$ Vgl. Georges Scelle Précis de droit des gens, Band 1, 1932, 69. - Zum Problem der „doppelten Loyalität" der (nationalen) Verwaltungen gegenüber dem nationalen und 
sprechen. Aus dieser Doppelrolle der staatlichen Verwaltung, die sich in allen drei Formen des Vollzugsföderalismus in ganz ähnlicher Weise zeigt, resultieren spezifische Interessen- und Loyalitätskonflikte, die man im Modell des zentralen Vollzugs nicht (oder nicht in dieser Form) kennt und die im traditionellen Allgemeinen Verwaltungsrecht nicht zureichend verarbeitet sind..$^{74}$

Im Zusammenhang mit diesen potenziellen Interessen- und Loyalitätskonflikten spielt die grundlegende Kategorie des Vertrauens ${ }^{75}$ eine zentrale Rolle - konkret: des Vertrauens in die Fähigkeit und Bereitschaft, nicht nur das jeweils „eigene“, sondern auch höherstufiges Recht vorgabengetreu zu vollziehen. Vertrauen ist nicht etwas fest Vorgegebenes, sondern eine veränderliche, dynamische Grösse. So war es z.B. im jungen schweizerischen Bundesstaat um die Beachtung der Bundesvorgaben durch die Kantone (bzw. ihre Verwaltungen) noch nicht so gut bestellt wie heute. Entsprechend gab es im 19. Jahrhundert eine (im Vergleich zu heute) recht lebhafte Praxis der Bundesaufsicht. ${ }^{76}$ Nach dem Ende der bundesstaatlichen „Flegeljahre“ konnte man die Zügel nach und nach lockern. Heute ist das Vertrauen intakt und die Bundesaufsicht kaum mehr sichtbar7 ${ }^{77}$ dies mit der - auf den ersten Blick paradox anmutenden - Konsequenz, dass man heute in der Europäischen Union in etlichen Bereichen die Mitgliedstaaten viel schärfer kontrolliert als es in der Schweiz im Rahmen der Bundesaufsicht die Kantone zu gewärtigen haben, obwohl man doch, ganz im Gegenteil, erwarten würde, dass die Aufsicht in einem Bundesstaat intensiver ist als die Aufsicht im Rahmen der Europäischen Union.

Hier kündigt sich eine Grundschwierigkeit des dezentralen Vollzugs im völkerrechtlichen Verhältnis an. Solange das gegenseitige Vertrauen in die Fähigkeit und die Bereitschaft, die gemeinsamen Regeln vorgabengetreu zu vollziehen, fehlt oder nur schwach ausgebildet ist, besteht ein erhöhter Bedarf nach rigorosen Kontrollmöglichkeiten und -mitteln.

dem europäischen Recht Eberhard Schmidt-Aßmann Allgemeines Verwaltungsrecht in europäischer Perspektive, Zeitschrift für Öffentliches Recht 55 (2000), 159 (168). Vgl. auch Kadelbach Allgemeines Verwaltungsrecht (Fn. 16), 17; Sydow Verwaltungskooperation (Fn. 39), 2f. (je mit Hinweisen).

74 Vgl. vorne Fn. 52.

75 Vgl. Röhl Akkreditierung (Fn. 63), 45 ff. (mit Hinweis auf Art. IV Sec. 1 der USVerfassung).

${ }^{76}$ Vgl. Walther Burckhardt Kommentar der schweizerischen Bundesverfassung vom 29. Mai 1874, 3. Aufl. 1931, 684 ff. (mit weiteren Hinweisen).

${ }^{7}$ Vgl. Giovanni Biaggini Bundesverfassung der Schweizerischen Eidgenossenschaft. Kommentar, 2007, Rn. 15 ff. zu Art. 49. 
Doch wird sich für deren Bereitstellung der nötige Konsens auf internationaler Ebene nicht so leicht finden lassen.

Umso mehr sind Praxis und Rechtswissenschaft herausgefordert und aufgerufen, nach Möglichkeiten zu suchen, wie man im internationalen Verhältnis die Qualität und Verlässlichkeit des nationalen Vollzugs verbessern und das Vertrauen in die nationalen Vollzugsstrukturen stärken kann. Einen Beitrag zur Vertrauensbildung können internationale „Behörden-Netzwerke“ leisten. Diese Figur wird zu Recht vielfach als problematisch eingestuft ${ }^{78}$, sie sollte jedoch - im Lichte entsprechender bundesstaatlicher und europäischer Erfahrungen - nicht pauschal abgelehnt werden.

Der Vertrauensbildung dient auch die Schaffung möglichst günstiger structures d'accueil (wie man in der französischsprachigen Schweiz sagt ${ }^{79}$ oder „Empfangsstrukturen“ für das Völkerrecht im nationalen Bereich. Eine dem Monismus verpflichtete Rechtsordnung (wie die schweizerische oder jene der EG ${ }^{80}$ ) ist dem Völkerrecht gegenüber offener und empfänglicher als ein dualistisches System. ${ }^{81}$ Räumt eine Rechtsordnung dem Völkerrecht grundsätzlich Vorrang vor dem Landesrecht ein - in diesem Sinn das Grundsatzbekenntnis in Art. 5 der schweizerischen Bundesverfassung ${ }^{82}$-, so bildet sich leichter Vertrauen. Eine spezifische demokratische Abstützung von völkerrechtlichen Verträgen, wie sie in der Schweiz durch das fakultative Staatsvertragsreferendum bewirkt wird (Art. $141 \mathrm{BV})^{83}$, stärkt die innerstaatliche Stellung des Völkerrechts beträchtlich und damit auch dessen Vollzug.

\footnotetext{
${ }^{78}$ Für einen Überblick über mögliche Einwände vgl. Slaughter New World Order (Fn. 40), $217 \mathrm{ff}$.

${ }^{79}$ Näher Olivier Jacot-Guillarmod Le juge national face au droit européen, 1993, insb. $194 \mathrm{ff}$.

${ }_{80}$ Vgl. etwa Roland Bieber/Astrid Epiney/Marcel Haag Die Europäische Union, 7. Aufl. 2006, §33 Rz. 29f. Zur Relativierung dualistischer und monistischer Theorien durch Übernahme von Völkerrecht in das Gemeinschaftsrecht vgl. Tietje Internationalisiertes Verwaltungshandeln (Fn. 1), $568 \mathrm{ff}$.

${ }_{81}$ Zur Problematik des Dualismus im Lichte der Entwicklung eines „Internationalen Verwaltungsrechts“ vgl. auch Ruffert Perspektiven, in: Möllers/Voßkuhle/Walter (Hrsg.) (Fn. 6), 413.

82 Dazu Bundesgericht, Urteil vom 26. Juli 1999, BGE 125 II 417 (425); Bundesgericht, sentenza del 14 aprile 2005, BGE 131 II 352 (355); René Rhinow Grundzüge des Schweizerischen Verfassungsrechts, 2003, 566ff.; Daniel Thürer Verfassungsrecht und Völkerrecht, in: ders./Jean-François Aubert/Jörg Paul Müller (Hrsg.), Verfassungsrecht der Schweiz, 2001, § 11, Rn. $26 \mathrm{ff}$.

${ }^{83} \mathrm{Vgl}$. Valentin Zellweger Die demokratische Legitimation staatsvertraglichen Rechts, in: Thomas Cottier u.a. Der Staatsvertrag im schweizerischen Verfassungsrecht, 2001,
} 
Neben solchen eher graduellen Unterschieden gibt es auch einen bedeutenden prinzipiellen Unterschied: Auf völkerrechtlicher Ebene fehlt ein fester allgemeiner Ordnungsrahmen, wie ihn im Bundesstaat die Bundesverfassung und in der Europäischen Union das (geschriebene und ungeschriebene) Primärrecht bilden - je verstärkt durch ein Justizsystem, an dessen Spitze eine für einheitliche Rechtsanwendung sorgende Instanz steht (in der EU der EuGH ${ }^{84}$, in der Schweiz das Bundesgericht ${ }^{85}$ ). Ein solcher „Schlussstein“ im Gefüge der Justiz fehlt bei dezentralem Vollzug von Völkerrecht gewöhnlich ${ }^{86}$ - dies mit weit reichenden Folgen für das zentrale Anliegen der gleichgerichteten Auslegung und des gleichmässigen Vollzugs. Immerhin können nationale Gerichte (im EU-Rahmen der EuGH) einen wichtigen Beitrag zum Ausgleich des strukturellen Defizits leisten. ${ }^{87}$ Dies setzt allerdings, erstens, voraus, dass es um Normen geht, die für eine unmittelbare Anwendung in Betracht kommen ${ }^{88}$, und, zweitens, dass die Gerichte ihre traditionelle Zurückhaltung in diesem Punkt überdenken und die unmittelbare Anwendbarkeit nicht vorschnell verneinen.

Im völkerrechtlichen dezentralen Vollzug kommt erschwerend hinzu, dass die teilnehmenden Staaten (deren Kreis im Prinzip variabel ist) oft durch unterschiedliche Gemeinwohl-Verständnisse und „Verwaltungskulturen" geprägt sind. Zudem stösst man, häufiger als in Bundesstaaten $^{89}$, auf Asymmetrien; dies mitunter auch in Verträgen zwischen der

254 (insb. $281 \mathrm{ff}$ ); Luzius Wildhaber Kontrolle der auswärtigen Gewalt, VVDStRL 56 (1997), 71. - Nach Ablehnung des Beitritts zum Europäischen Wirtschaftsraum (6. 12. 1992) genehmigten die Schweizer Stimmberechtigten die sog. Bilateralen Verträge I mit der EG/EU (21. 5. 2000), das Abkommen betreffend Assoziierung zu "Schengen“ und "Dublin“ (5. 6. 2005) sowie die Ausdehnung des Personen-Freizügigkeitsabkommens (vgl. Fn. 99) auf die zehn neuen EU-Mitgliedstaaten (25. 9. 2005). Am 3. März 2002 stimmten Volk und Stände dem Beitritt der Schweiz zur UNO zu (Volksinitiative; vgl. Art. 197 Ziffer 1 BV).

${ }^{84}$ Wenn auch bloss via Vorabentscheidungsverfahren (Art. 234 EGV), d.h. ohne den für Bundesstaaten typischen direkten Instanzenzug.

${ }^{85}$ Vgl. Pierre Tschannen Staatsrecht der Schweizerischen Eidgenossenschaft, 2. Aufl. $2007,512 \mathrm{ff}$.

${ }^{86}$ Im WTO-Recht ist ein Streitschlichtungsverfahren vorgesehen, doch können dort nur (WTO-Mitglied-)Staaten, nicht aber Private als Streitparteien auftreten.

${ }^{87}$ Zur Bedeutung nationaler Gerichte vgl. auch Kingsbury/Krisch/Stewart Emergence (Fn. 10), 15 (55). - Zur informellen Koordination der Rechtsprechung vgl. auch den Beitrag von Georg Nolte in diesem Band (Ziffer II.2. mit These 8).

${ }^{88}$ Darauf ist bei der Formulierung entsprechender völkerrechtlicher Rechtsakte (vgl. auch Fn. 109) zu achten.

${ }^{89}$ Vgl. Francesco Palermo/Rudolf Hrbek (Hrsg.) Auf dem Weg zu asymmetrischem Föderalismus?, 2007. 
Schweiz und der EU (EG) - gewöhnlich zu Lasten der Schweiz. ${ }^{90} \mathrm{Um}$ gekehrt wird das vertraglich sehr eng mit der Schweiz verflochtene Fürstentum Liechtenstein von „Bern“ nicht immer sehr pfleglich behandelt, in der Gesetzgebung manchmal wie ein 27. Kanton ${ }^{91}$ (was man zum Glück für die Schweiz - in „Brüssel“ kaum wahrnimmt).

Kurz: Der im Völkerrecht gründende dezentrale Vollzug oder „völkerrechtliche Vollzugsföderalismus“ entpuppt sich im Vergleich zum bundesstaatlichen bzw. gemeinschaftsrechtlichen Gegenstück als ein noch viel ausgeprägteres Mängelwesen.

\section{Ansätze zum Ausgleich des strukturellen Defizits in der völkerrechtlichen Praxis}

Die völkerrechtliche Praxis nimmt das strukturelle Defizit nicht einfach hin. Mangel macht erfinderisch: Ein illustratives Beispiel ist das WTO-Übereinkommen über das öffentliche Beschaffungswesen von $1994^{92}$, in der schweizerischen Rechtspraxis sehr präsent, in der EU durch das europäische Vergaberecht in den Hintergrund gedrängt. ${ }^{93}$ In der Sache geht es um die Verwirklichung eines zentralen Anliegens, nämlich um die Nicht-Diskriminierung von Bietern aus anderen Vertragsstaaten. Das WTO-Übereinkommen besteht im Wesentlichen aus diesem Zweck dienenden Vorgaben betreffend das nationale Vergabeverfahren ${ }^{94}$

90 Hauptbeispiel ist das Abkommen vom 21. Juni 1999 zwischen der Schweizerischen Eidgenossenschaft und der Europäischen Gemeinschaft über den Luftverkehr (SR 0.748.127.192.68), welches die Fusionskontrolle in die Zuständigkeit der Organe der Gemeinschaft legt (Art. 11). Dazu Regula Dettling-Ott Das bilaterale Luftverkehrsabkommen der Schweiz und der EG, in: Thürer u.a. (Hrsg.) (Fn. 27), $491 \mathrm{ff}$.

91 Vgl. z.B. Art. 4 Abs. 1 bis der bundesrätlichen Verordnung vom 23. August 2000 über das Fahrberechtigungsregister (SR 741.53): „Die Verkehrspolizeien der Schweiz und des Fürstentums Liechtenstein [...] tragen die Abnahme eines Führerausweises sowie an Ort und Stelle ausgesprochene Fahrverbote [...] unverzüglich im [Fahrberechtigungsregister] ein."

${ }^{2} \mathrm{Vgl}$. Thomas Cottier/Matthias Oesch International Trade Regulation, 2005, 1036ff.; Götz J. Göttsche Öffentliches Beschaffungswesen, in: Meinhard Hilf/Stefan Oeter, WTO-Recht, 2005, $467 \mathrm{ff}$;; Andreas $R$. Ziegler De l'inévitable internationalisation de la procédure administrative: l'exemple des marchés publics, FS Pierre Moor, 2005, 623.

${ }^{93}$ Zum europäischen Vergaberecht und seinem welthandelsrechtlichen Hintergrund vgl. Thomas Puhl Der Staat als Wirtschaftssubjekt und Auftraggeber, VVDStRL 60 (2001), 456 (463f.); Hans-Joachim Prieß Das öffentliche Auftragswesen in der Europäischen Union, 3. Aufl. 2005.

$94 \mathrm{Vgl}$. Art. VII-XVI GPA (mit detaillierten Vorschriften betreffend öffentliche Bekanntmachung, Leistungsbeschreibung, Fristen, Angebotsprüfung, Zuschlagserteilung u.a.m.). 
und das nationale Rechtsschutzverfahren ${ }^{95}$. Die Vorgaben sind zum Teil so detailliert, dass das schweizerische Bundesgericht seine traditionelle Zurückhaltung in Bezug auf die unmittelbare Anwendbarkeit von WTORecht beim Beschaffungsabkommen abgelegt hat.96 Ein ergänzendes bilaterales Abkommen zwischen der Schweiz und der EG ${ }^{97}$ verpflichtet die beiden Vertragsparteien, im Beschaffungswesen je eine unabhängige Überwachungsbehörde einzusetzen, welche befugt ist, „gegen die beteiligten Vergabestellen ein Verfahren einzuleiten oder verwaltungstechnische oder rechtliche Schritte zu unternehmen“ (Art. 8).98 Das (Personen-) Freizügigkeitsabkommen Schweiz-EG ${ }^{99}$ verpflichtet die Vertragsparteien, dafür zu sorgen, dass betroffene Personen sich an ein nationales Gericht wenden können (Art. 11).100 Die Liste mit Beispielen liesse sich leicht verlängern, etwa um die Aarhus-Konvention mit völ-

95 Vgl. Art. XX GPA (mit detaillierten Mindestanforderungen betreffend den Kreis der Rechtsschutzberechtigten, die Stellung der Beschwerdeinstanz, das Beschwerdeverfahren u.a.m.). - Auf internationaler Ebene wacht ein Ausschuss für das öffentliche Beschaffungswesen über das ordnungsgemässe Funktionieren des GPA. Bei Streitigkeiten zwischen Vertragsparteien kann das WTO-Streitbeilegungsverfahren in Gang gesetzt werden (vgl. Art. XXII GPA). Die Praxis dazu ist noch recht spärlich. Vgl. Göttsche Beschaffungswesen, in: Hilf/Oeter (Fn. 92), $486 \mathrm{ff}$.

$96 \mathrm{Vgl}$. Bundesgericht, Urteil vom 26. 6. 2000, in: Schweizerisches Zentralblatt für Staats- und Verwaltungsrecht (ZBl) 2001, 215 (216f.). Vgl. auch Lukas Engelberger Die Frage der unmittelbaren Anwendbarkeit von WTO-Recht in der Rechtsprechung des schweizerischen Bundesgerichts, Schweizerische Zeitschrift für internationales und europäisches Recht 2004, 155 (178ff.).

97 Abkommen vom 21. Juni 1999 zwischen der Schweizerischen Eidgenossenschaft und der Europäischen Gemeinschaft über bestimmte Aspekte des öffentlichen Beschaffungswesens (SR 0.172.052.68). Näher Giovanni Biaggini Das Abkommen über bestimmte Aspekte des öffentlichen Beschaffungswesens, in: Daniel Thürer u.a. (Hrsg.) (Fn. 27), 651.

98 In der EG ist es die Kommission, in der Schweiz die Kommission „Beschaffungswesen“ Bund-Kantone (KBBK), die sich paritätisch aus Vertretern des Bundes und der Kantone zusammensetzt.

99 Abkommen vom 21. Juni 1999 zwischen der Schweizerischen Eidgenossenschaft einerseits und der Europäischen Gemeinschaft und ihren Mitgliedstaaten andererseits über die Freizügigkeit (SR 0.142.112.681).

100 Gemäss Art. 16 ist bei der Auslegung von Begriffen, die aus dem Gemeinschaftsrecht stammen, „die einschlägige Rechtsprechung des Gerichtshofs der Europäischen Gemeinschaften vor dem Zeitpunkt der Unterzeichnung“ (21. Juni 1999) zu berücksichtigen. Die Rechtsprechung des Bundesgerichts zieht auch nach dem Stichtag ergangene Urteile des EuGH heran. Vgl. Bundesgericht, Urteil vom 4. November 2003, BGE 130 II 1 (10f.). 
kerrechtlichen Vorgaben betreffend Beteiligungs- und Beschwerderechte in umweltbezogenen Verfahren. ${ }^{101}$

In vorsichtiger Verallgemeinerung kann man festhalten, dass die völkerrechtliche Praxis stark auf verfahrensrechtliche Vorgaben an die Adresse der Vertragsstaaten setzt ${ }^{102}$ sowie auf eine Instrumentalisierung des nationalen Rechtsschutzsystems. Es sind dies Mittel und Methoden, die man aus dem bundesstaatlichen und europäischen Vollzugsföderalismus kennt - wobei der Erfolg im völkerrechtlichen Kontext, aus den genannten Gründen, nicht ohne weiteres garantiert ist.

Kaum präsent sind dagegen Parallelen zum Instrumentarium der Bundesaufsicht (wie generelle Weisungen, besondere Beschwerderechte). Dies überrascht nicht sonderlich. Immerhin könnte man die Bildung von Netzwerken aus nationalen Aufsichtsbehörden (auch) als Versuch deuten, im Interesse der Einhaltung gemeinsamer Standards eine Art informelle "kollektive Aufsicht" einzurichten, die sich - mit begrenzten Mitteln - für die Einhaltung gemeinsamer Standards einsetzt, als eine Art Ersatz für (noch) fehlende echte Aufsichtsmechanismen.103 Die - ambivalente - Figur des "Behörden-Netzwerks“ erscheint in einem etwas milderen Licht, wenn und soweit sie einen Beitrag zur Gewährleistung einheitlicher Rechtsanwendung leistet, d.h. das strukturelle Defizit bei den Rechtsverwirklichungsstrukturen auszugleichen hilft.

Eine differenzierende Beurteilung verdient unter dem Aspekt eines gleichmässigen, wirksamen Verwaltungsvollzugs auch der Ansatz, nationale Aufsichtsinstanzen (via übergeordnetes Recht) mit einem gewissen Grad an Unabhängigkeit auszustatten (Beispiel: Beschaffungsabkommen) und auf diese Weise partiell aus der nationalen Verwaltung heraus-

$101 \mathrm{Vgl}$. (aus schweizerischer Sicht) Daniela Thurnherr Öffentlichkeit und Geheimhaltung von Umweltinformationen, 2003, $75 \mathrm{ff}$; (aus deutscher Sicht) Christian Walter Internationalisierung des deutschen und europäischen Verwaltungsverfahrens- und Verwaltungsprozessrechts - am Beispiel der Århus-Konvention, Europarecht 2005, 302.

${ }^{102}$ In diesem Sinne auch WTO Appellate Body, Untied States - Import Prohibition of Certain Shrimp and Shrimp Products (WT/DS58/AB/R), angenommen am 6. November 1998 (welthandelsrechtliche Vorgaben an nationale Entscheidungsverfahren). Vgl. dazu Sabino Cassese Global Standards for National Administrative Procedure, Law and Contemporary Problems 68 (2005), 109.

${ }^{103}$ Die informelle Abstimmung scheint im Bereich der Finanzmarktaufsicht zum Teil so erfolgreich zu sein, dass die fehlende rechtliche Verbindlichkeit der gemeinsamen Standards (vgl. Fn. 31) nicht nachteilig ins Gewicht fällt. Vgl. Möllers Behördenkooperation (Fn. 29), 366. - Zur Kooperation von nationalen Aufsichtsbehörden als Form der transnationalen „Aufsichtskoordination“ Schmidt-Aßmann Internationalisierung (Fn. 35), 161. 
zulösen. 104 Unter rechtsstaatlich-demokratischem Blickwinkel ist der Vorgang nicht unproblematisch. Man sollte aber bei der rechtlichen Würdigung berücksichtigen, dass es sich um ein auf nationaler Ebene praktiziertes und grundsätzlich bewährtes Mittel handelt, um möglichen Interessenkonflikten zu begegnen. „Herauslösen aus der Verwaltung“ heisst im Übrigen nicht: Herauslösen aus parlamentarischen und justiziellen Kontrollzusammenhängen. Im hier interessierenden völkerrechtlichen Kontext kommt hinzu, dass damit das Vertrauen in einen unvoreingenommenen, vorgabengetreuen nationalen Verwaltungsvollzug wesentlich gestärkt werden kann. Und dies wiederum fördert die Bereitschaft, sich überhaupt erst auf ein gemeinsames - internationales rechtliches Regime einzulassen.

Dem Ausgleich struktureller Defizite können auch allgemeine Rechtsgrundsätze und Auslegungsmaximen dienen. Zu denken ist etwa an eine Verallgemeinerung des in der EU geläufigen effet-utile-Prinzips ${ }^{105}$, an eine konsequente völkerrechtskonforme Interpretation nationalen Rechts oder an die schon erörterte grosszügigere Bejahung der unmittelbaren Anwendbarkeit völkerrechtlicher Regelungen. ${ }^{106}$ Dies kann hier nicht weiter vertieft werden.

Die Gegenüberstellung der Rechtsverwirklichungsstrukturen - bundesstaatlich, gemeinschaftsrechtlich, völkerrechtlich - zeigt: Ein prinzipieller Unterschied und zugleich eine Hauptschwierigkeit beim dezentralen Vollzug völkerrechtlicher Vorgaben ist das Fehlen eines festen allgemeinen Rechtsrahmens, wie ihn eine Bundesverfassung oder das Primärrecht bieten. ${ }^{107}$ Zugleich wird deutlich, wie stark der dezentrale Verwaltungsvollzug im Bundesstaat bzw. in der EU durch den allgemeinen rechtlichen Rahmen geprägt und vor allem auch gestützt wird.

$104 \mathrm{Zu}$ Herauslösungstendenzen im Bereich des Produktesicherheitsrechts vgl. Röhl Akkreditierung (Fn. 63), 23ff.; ders. Verantwortung und Effizienz in der Mehrebenenverwaltung, DVB1 2006, 1070 (1078f.).

105 Dazu etwa Rudolf Streinz Europarecht, 7. Aufl. 2005, Rn. 570, 798.

$106 \mathrm{Vgl}$. Ziffer III.2. - Ob die Zeit für eine Verallgemeinerung des aus dem Gemeinschaftsrecht bekannten Herkunftsortsprinzips (Cassis-de-Dijon-Prinzip) reif ist, erscheint fraglich, denn das hierfür erforderliche Vertrauen in die Qualität anderer Rechtssysteme bzw. in die Zuverlässigkeit des Verwaltungsvollzugs (vgl. Ziffer III.2.) kann ausserhalb der EU nicht ohne weiteres vorausgesetzt werden. Zu optimistisch Breining-Kaufmann Internationales Verwaltungsrecht (Fn. 7), 69.

107 In diesem Sinne auch Sabino Cassese Administrative Law Without the State? The Challenge of Global Regulation, N.Y.U. Journal of International Law and Politics 37 (2005), 663 (687): „The constitutional framework holding up domestic administrative law is lacking in the global arena." 


\section{Perspektiven}

\section{Aufgaben der Rechtswissenschaft}

Da im internationalen Kontext ein fester allgemeiner Ordnungsrahmen und (gewöhnlich) eine „,krönende“ Justizinstanz fehlt, ist die Praxis in besonderem Masse auf Unterstützung durch die Rechtswissenschaft angewiesen. Die vornehmste Aufgabe unserer Wissenschaft ist es, darauf hinzuwirken, dass rechtsstaatlich-demokratische Grundanliegen respektiert werden. Dies gilt selbstverständlich auch für die hier interessierende internationale Dimension des Verwaltens.

Die Rechtswissenschaft sollte indes ihre Aufmerksamkeit nicht allein auf Rechtsschutz- und Legitimationsfragen richten, sondern auch eine aktive Rolle übernehmen bei der Suche nach Möglichkeiten, die strukturellen Defizite des dezentralen Völkerrechtsvollzugs auszugleichen. Diese Aufgabe mag vielleicht nicht so prestigeträchtig sein, sie ist aber sehr wichtig, denn ohne Unterstützung und kritische Begleitung durch die Lehre wird sich die Praxis schwer tun - die Verwaltungspraxis, aber auch die Praxis der parlamentarisch-demokratischen Verwaltungskontrolle.

Kontextbedingt zeigen sich für die Rechtswissenschaft im hier interessierenden Bereich verschiedene Besonderheiten. So muss der Blick stets in zwei Richtungen gehen: durch die völkerrechtliche Brille auf das verwaltungsrechtliche Terrain, durch die verwaltungsrechtliche Brille auf das völkerrechtliche Terrain. ${ }^{108}$ Im ebenenübergreifenden Verwaltungsvollzug müssen, mehr als sonst, Interessengegensätze verarbeitet werden. Vorschläge für die Ausgestaltung von Vollzugsstrukturen müssen auf eine sorgfältige Interessenanalyse abgestützt sein, wobei nicht nur Gegensätze interessieren, sondern auch ein allfälliger „Interessengleichklang " (Stichwort: Aktivierung der Bürgerinnen und Bürger, Instrumentalisierung des Rechtsschutzsystems).

Weiter gilt es, für den Bereich der „Internationale(n) Rechtssetzung " 109 (Aushandeln von Vereinbarungen, Setzen von Sekundärrecht) vermehrt Erkenntnisse der Gesetzgebungslehre fruchtbar zu machen. Ein zentrales Anliegen ist die systematische Prüfung der Vollzugstauglichkeit von geplanten Regelungen, wie sie in der Schweiz - gerade mit

108 Ähnlich Möllers Internationales Verwaltungsrecht, in: ders./Voßkuhle/Walter (Hrsg.) (Fn. 6), 4 (,von zwei Seiten her denken“). Anders gelagert ist die Unterscheidung zwischen einem bottom-up und einem top-down approach bei Kingsbury/Krisch/Stewart Emergence (Fn. 10), 15 (55 ff.).

109 Georg Müller Elemente einer Rechtssetzungslehre, 2. Aufl. 2006, 247 ff. (Titel des abschliessenden 4 . Teils). 
Blick auf den dezentralen Vollzug von Bundesrecht - zu den traditionellen Obliegenheiten der gesetzesvorbereitenden Instanzen gehört. ${ }^{110}$

Um von den Erfahrungen auf bundesstaatlicher und europäischer Ebene profitieren zu können, ist es sodann wichtig, diese Erfahrungen in geeigneter, anschlussfähiger Weise aufzuarbeiten: Es gilt, das nationale (und europäische) Verwaltungsrecht auf exportfähige Gedanken und Rechtsfiguren hin zu untersuchen und deren Vor- und Nachteile aufzuzeigen, ohne dabei in ,juristischen Patriotismus" zu verfallen (Stichwort: Wettbewerb der Lösungsansätze). Auf der Suche nach Bauelementen für sachgerechte Lösungen ist nicht nur ein horizontaler Ländervergleich gefragt, sondern auch, wie hier gezeigt wurde, ein vertikaler Vergleich der verschiedenen Varianten dezentralen Vollzugs: bundesstaatlich, gemeinschaftsrechtlich, völkerrechtlich. ${ }^{111}$ Es wird hier einmal mehr deutlich, dass die „Entwicklung eines Internationalen Verwaltungsrechts" eine Aufgabe nicht allein der Verwaltungsrechtswissenschaft, sondern der Wissenschaft des Öffentlichen Rechts insgesamt ist, unter Einschluss der Staatsrechts-, Europarechts- und Völkerrechtslehre. Der Vortragstitel ist in dieser Hinsicht mit gutem Grund offen formuliert („Aufgabe der Rechtswissenschaft“).

\section{Zur Einordnung des "Internationalen Verwaltungsrechts"}

Vor diesem Hintergrund wird es niemanden überraschen, wenn hier die These vertreten wird, dass die Entwicklung eines Systems des „Internationalen Verwaltungsrechts" kein vorrangiges Anliegen ist. Dies nicht etwa nur, weil es verfrüht wäre oder weil es schade wäre, kreatives Denken in ein System zu zwängen, sondern auch aus grundsätzlichen Überlegungen, die mit der Beschaffenheit des „Internationalen Verwaltungsrechts" zusammenhängen. Das „Internationale Verwaltungsrecht", wie es hier verstanden und (ausschnittweise) entwickelt wurde, muss in einem demokratisch gestützten Prozess wachsen. Beim „Internationalen Verwaltungsrecht" handelt es sich nicht um ein mehr oder weniger eigenständiges Rechtsgebiet und auch nicht um die Zusammenfassung verschiedener Teilgebiete (Summe aus Internationalem Steuerrecht,

${ }_{10}$ Vgl. Art. 141 Abs. 2 Bst. d ParlG (Fn. 50), zurückgehend auf die Parlamentarische Initiative 96.456 „Verbesserung der Vollzugstauglichkeit von Massnahmen des Bundes“, eingereicht von Ständerat René Rhinow (vgl. Bundesblatt 1999, 2761).

${ }^{111} \mathrm{Zu}$ verschiedenen Blickwinkeln des Vergleichens allgemein Christoph Möllers Theorie, Praxis und Interdisziplinarität in der Verwaltungsrechtswissenschaft, Verwaltungsarchiv 93 (2002), 22 (46ff.: „Komparative Verwaltungsrechtswissenschaft“). Vgl. auch Giulio Napolitano (Hrsg.) Diritto amministrativo comparato, 2007. 
Sozialrecht, Umweltrecht usw.).112 Unter dem Titel „Internationales Verwaltungsrecht" geht es vielmehr darum, jene Rechtsfiguren und Strukturen $\mathrm{zu}$ untersuchen und zu entwickeln, welche die dezentrale Verwirklichung von völkerrechtlichen Regeln mit verwaltungsrechtlichem Einschlag organisieren - dies mit Blick auf das Ziel eines vorgabengetreuen, gleichmässigen (und auch sonst rechtsstaatlich und demokratisch einwandfreien) Verwaltungsvollzugs.

Unter dem Aspekt der involvierten Rechtsquellen handelt das „Internationale Verwaltungsrecht" von Regelungen, die teils dem Völkerrecht, teils dem nationalen (allenfalls europäischen ${ }^{113}$ ) Recht zuzuordnen sind. 114 Eine Aufspaltung in einen nationalen und einen internationalen Teil sollte vermieden werden.115 Das Nebeneinander eines deutschen, österreichischen, schweizerischen, liechtensteinischen „Internationalen Verwaltungsrechts" kann kein erstrebenwertes Ziel sein. Entsprechenden Entwicklungen kann man leichter entgegenwirken, wenn man das "Internationale Verwaltungsrecht" nicht als ein primär am positiven Recht orientiertes Rechtsgebiet konzipiert, sondern als eine in erster Linie an theoretischen Grundlagen und Grundfragen interessierte Disziplin - im Sinne von Allgemeinen Lehren.

112 Entsprechend wird sich das "Internationale Verwaltungsrecht" wohl kaum als eigenständiges Unterrichtsfach (mit eigenen Lehrbüchern und Prüfungen) etablieren können.

${ }^{113}$ Ein Ausblenden des Europäischen Verwaltungsrechts aus dem „Internationalen Verwaltungsrecht" ist ebenso wenig angezeigt wie ein prinzipielles Ausklammern des Kollisionsrechts (vgl. Fn. 22).

114 Zur Rechtsquellenvielfalt im Bereich des „Internationalen Verwaltungsrechts“ vgl. auch Ruffert Perspektiven, in: Möllers/VoBkuhle/Walter (Hrsg.) (Fn. 6), $406 \mathrm{ff}$. - Ungeachtet der Annäherungen (vgl. Ziffer I.1.) gilt es die einzelnen Rechtsquellen weiterhin zu unterscheiden (problematisch daher Krisch/Kingsbury Global Governance (Fn. 4), 11: "the blurred distinction between domestic and international law"). - Es ist kein Zufall, dass das (wieder)erwachte Interesse an der Entwicklung eines „Internationalen Verwaltungsrechts" in eine Zeit fältt, in der man sowohl mit Blick auf das Völkerrecht als auch mit Blick auf das Verwaltungsrecht viel von Strukturwandel spricht. Man kann das "Internationale Verwaltungsrecht" (bzw. die wissenschaftliche Auseinandersetzung damit) auch als eine Art Seismograph auffassen, welcher Wandlungen bzw. Veränderungsdiskurse registriert, lokalisiert und sichtbar macht.

115 Dies scheint beim Internationalen Wirtschaftsrecht geglückt zu sein. Vgl. Herdegen Internationales Wirtschaftsrecht (Fn. 9), §1 Rz 6. - Die rechtsquellen-übergreifende Konzeption einer Rechtsdisziplin ist nichts Ungewöhnliches, wie ein Blick auf das Allgemeine Verwaltungsrecht zeigt. Zur Vielfalt der Rechtsquellen vgl. z.B. Häfelin/Müller/Uhlmann Verwaltungsrecht (Fn. 14), Rn. 97 ff.; Ruffert Rechtsquellen, in: HoffmannRiem/Schmidt-ABmann/Voßkuhle (Hrsg.), GVwR I (Fn. 11), § 17, Rz. 30 ff. (wo Gemeinschaftsrecht und Völkerrecht an der Spitze stehen). 
Inhaltlich und strukturell besteht eine grosse Nähe zum Allgemeinen Verwaltungsrecht ${ }^{116}$, sofern man das Allgemeine Verwaltungsrecht nicht reduziert auf jene Regeln des positiven Verwaltungsrechts, die gleichsam „vor die Klammer gezogen“ wurden. Bei den Fragen des „Internationalen Verwaltungsrechts" geht es letztlich um besonders gelagerte - nämlich: internationale - Aspekte der Allgemeinen Lehren des Verwaltungsrechts. Es empfiehlt sich daher, das „Internationale Verwaltungsrecht“ in die Allgemeinen Lehren des rechtsstaatlich-demokratischen Verwaltungsrechts zu integrieren. Im Übrigen sollte man die sich abzeichnende Herausbildung eines „Internationalen Verwaltungsrechts“ zum Anlass nehmen, der Verwaltungsrechtstheorie und der Verwaltungsrechtsvergleichung generell noch mehr Aufmerksamkeit zu schenken.

$116 \mathrm{Zu}$ den Funktionen des Allgemeinen Verwaltungsrechts Schmidt-Aßmann Ordnungsidee (Fn. 36), 3 ff., wo (mit Blick auf die Europäisierung) besonders auch die ,rezeptionsleitende Funktion“ herausgearbeitet wird (6). 
Leitsätze des 2. Berichterstatters über:

\section{Die Entwicklung eines Internationalen Verwaltungsrechts als Aufgabe der Rechtswissenschaft}

\section{I. „Internationales Verwaltungsrecht“ als Phänomen \\ 1. Ausgangspunkte und Herangehensweisen}

1. Völkerrecht zeigt inhaltlich vermehrt verwaltungsrechtliche Züge, Verwaltungsrecht hat zunehmend einen völkerrechtlichen Hintergrund (welcher im EU-Raum mitunter durch Gemeinschaftsrecht verdeckt wird). Auf diese Entwicklung kann aus rechtswissenschaftlicher Sicht auf zwei Arten reagiert werden: durch Integration internationaler Aspekte in eine bestehende Disziplin oder durch Verselbstständigung. Unter dem Titel "Internationales Verwaltungsrecht“ deuten sich Verselbstständigungstendenzen an.

\section{Begriffliche Klärungen}

2. Mehr als andere Rechtssprachen (z.B. ital.: diritto amministrativo internazionale / diritto internazionale amministrativo) ist die deutsche Rechtssprache mit Mehrdeutigkeiten konfrontiert. Bis vor kurzem galt das kollisionsrechtliche Begriffsverständnis als „das geläufige“ („Internationales Verwaltungsrecht" als Verwaltungskollisionsrecht, in umstrittener Parallele zum Internationalen Privatrecht, IPR). In jüngerer Zeit wird unter dem Titel „Internationales Verwaltungsrecht" die Annäherung von Völkerrecht und Verwaltungsrecht erörtert (in Anlehnung an eine ältere, im 19. Jahrhundert wurzelnde Begriffstradition).

3. Das neuere Begriffsverständnis ist nicht ideal, aber zweckmässig und besser geeignet als begriffliche Alternativen wie „globales Verwaltungsrecht“, "transnationales Verwaltungsrecht“, „Verwaltungsvölkerrecht" oder „Völkerverwaltungsrecht". Einzubeziehen sind auch informelle Abstimmungsprozesse (unter Einschluss des sog. "autonomen Nachvollzugs"). 


\section{Grundprobleme eines "Internationalen Verwaltungsrechts“}

\section{Suche nach einem Ordnungsrahmen}

4. In der Literatur stehen heute im Zentrum: Fallstudien zu einzelnen Rechtsgebieten, Untersuchungen zu Querschnittsphänomenen (z.B. Verwaltungskooperation), zu Legitimations- und zu Rechtsschutzfragen oder $z u$ Einzelphänomenen. So wichtig diese Beiträge sind: Es bedarf eines Bezugsoder Ordnungsrahmens, um aus der Fülle des Materials gebietsübergreifende Erkenntnisse gewinnen zu können. Einprägsame Bilder und Schlagworte (wie "globaler Verwaltungsraum“, „Behörden-Netzwerk") lenken die Aufmerksamkeit auf bisher zu wenig beachtete Aspekte; der Beitrag zur Problemdurchdringung bleibt indes gering.

5. Weiter führt die (an das Tagungsmotto anknüpfende) Frage, worin die "Leistung" besteht, die man von Verwaltungsrecht und Verwaltungsrechtswissenschaft erwarten darf.

6. Das Aufgabenspektrum des Verwaltungsrechts geht über die Gewährleistung eines rechtsstaatlich einwandfreien, demokratisch abgestützten Verwaltungshandelns hinaus, wie ein Blick auf die Kriterien der Verwaltungskontrolle offenbart: Rechtmässigkeit, Zweckmässigkeit, Gleichmässigkeit, Wirksamkeit, Wirtschaftlichkeit (vgl. Art. 26 des eidg. Parlamentsgesetzes). Das Verwaltungsrecht organisiert den Prozess der verwaltungsmässigen Rechtsverwirklichung; es ist in weiten Teilen Rechtsverwirklichungsrecht.

\section{Strukturen der Rechtsverwirklichung im Bereich des Verwaltungsrechts}

7. Die administrative Rechtsverwirklichung ist schon im Fall der kleinen, überschaubaren, gut durchorganisierten Verwaltung eine anspruchsvolle Aufgabe, wie das grosse Arsenal an Instrumenten und Sicherungen zeigt (Weisungsbefugnisse, interne/externe Kontrollen usw.).

8. Die Schwierigkeiten nehmen erheblich zu, wenn die Rechtsverwirklichung - wie im bundesstaatlichen Vollzugsföderalismus - „gemeinwesenübergreifend" organisiert ist. Noch komplexer ist die Lage in der EU (dezentraler Vollzug des Gemeinschaftsrechts).

9. Besonders komplex sind die Verhältnisse bei der verwaltungsmässigen Umsetzung von völkerrechtlichen oder aus Völkerrecht abgeleiteten Vorgaben. Gerade hier ist das Bedürfnis nach gleichgerichteter Auslegung und Handhabung der gemeinsamen Regeln mitunter sehr ausgeprägt (Standortwettbewerb), ist die Distanz zwischen Regelungs- und Vollzugsebene besonders gross, ist das rechtliche Band sehr lose. 


\section{Folgerungen und Folgefragen}

10. Die drei Vollzugskonstellationen (Bundesstaat, EU, Völkerrecht) unterscheiden sich stark in Bezug auf die rechtlichen Rahmenbedingungen. Die verfolgten Ziele sind aber grundsätzlich dieselben (rechtmässiger, gleichmässiger Vollzug); die Probleme sind strukturell ähnlich. Dies legt es nahe, bundesstaatliche und gemeinschaftsrechtliche Erfahrungen fruchtbar zu machen.

\section{Grundfragen eines „Internationalen Verwaltungsrechts"}

\section{Zentrale und dezentrale Rechtsverwirklichung als Grundmodelle}

11. Im Bundesstaatsrecht haben sich Ersatz-Strategien entwickelt, die das im Vergleich zum zentralen Vollzug bestehende strukturelle Defizit auszugleichen helfen: Vorgaben an das Organisations- und Verfahrensrecht; Weisungen im Verhältnis Bund-Gliedstaaten; Instrumentalisierung des Rechtsschutzsystems für Zwecke der Bundesrechtsdurchsetzung (insb. Aktivierung der Bürgerinnen und Bürger; besondere Beschwerderechte für Verbände oder nationale Aufsichtsbehörden). Auf ähnliche Strategien trifft man im Gemeinschaftsrecht.

12. Diese Ansätze eignen sich als Ausgangsbasis für die Lösung der strukturell ähnlich gelagerten Probleme im völkerrechtlichen Kontext. Vorschnelle Analogien führen aber nicht zum Ziel.

\section{Gemeinsamkeiten und Unterschiede (im Vergleich zu den hergebrachten Typen)}

13. Verwaltungseinheiten haben (in allen drei vollzugsföderalistischen Konstellationen) nicht selten sowohl „heimisches" Recht als auch Regeln mit Ausgangspunkt im höherstufigen Recht zu vollziehen. Aus diesem "dédoublement fonctionnel " (Scelle) resultieren spezifische Interessen- und Loyalitätskonflikte, die im traditionellen Allgemeinen Verwaltungsrecht nicht zureichend verarbeitet sind.

14. Eine Grundschwierigkeit im völkerrechtlichen Kontext ist das schwache oder fehlende gegenseitige Vertrauen in die Fähigkeit und Bereitschaft, nicht nur "eigenes“, sondern auch höherstufiges Recht vorgabengetreu zu vollziehen. Einen Beitrag zur Vertrauensbildung können internationale „Behörden-Netzwerke“ leisten (auch wenn problematische Aspekte nicht übersehen werden dürfen). Der Vertrauensbildung dient die Schaffung möglichst günstiger „structures d'accueil“ für das Völkerrecht im nationalen Bereich 
(Verhältnis Völkerrecht-Landesrecht inkl. Vorrangfrage; demokratische Abstützung des Völkerrechts).

15. Auf völkerrechtlicher Ebene fehlt ein fester allgemeiner Ordnungsrahmen, wie ihn im Bundesstaat die Bundesverfassung und in der EU das Primärrecht bilden (je verstärkt durch ein Justizsystem mit einer für einheitliche Rechtsanwendung sorgenden Instanz an der Spitze). Erschwerend kommen hinzu: unterschiedliche Gemeinwohl-Verständnisse und "Verwaltungskulturen", mitunter auch Asymmetrien (z. B. im Verhältnis Schweiz-EU).

\section{Ansätze zum Ausgleich des strukturellen Defizits in der völkerrechtlichen Praxis}

16. Die Praxis nimmt das strukturelle Defizit nicht einfach hin. Beispiele wie das WTO-Übereinkommen über das öffentliche Beschaffungswesen oder die Aarhus-Konvention zeigen, dass man auf Mittel und Methoden setzt, die aus dem bundesstaatlichen und europäischen Vollzugsföderalismus bekannt sind (Vorgaben betreffend Verfahren und Rechtsschutz).

17. Parallelen zum Institut der Bundesaufsicht sind weniger präsent. Immerhin kann man Netzwerke aus nationalen Aufsichtsbehörden (auch) als Versuch deuten, im Interesse der Einhaltung gemeinsamer Standards eine informelle „kollektive Aufsicht" einzurichten. Eine differenzierende Beurteilung verdient unter dem Aspekt eines gleichmässigen, wirksamen Verwaltungsvollzugs auch der Ansatz, nationale Aufsichtsinstanzen (via übergeordnetes Recht) mit einem gewissen Grad an Unabhängigkeit auszustatten und partiell aus der nationalen Verwaltung herauszulösen.

18. Dem Ausgleich struktureller Defizite können auch allgemeine Rechtsgrundsätze und Auslegungsmaximen dienen (z.B. effet-utile-Prinzip, konsequente völkerrechtskonforme Interpretation).

19. Im Vergleich wird deutlich, wie stark der dezentrale Verwaltungsvollzug im Bundesstaat bzw. in der EU durch den allgemeinen rechtlichen Rahmen geprägt und gestützt wird.

\section{Perspektiven}

\section{Aufgaben der Rechtswissenschaft}

20. Da im internationalen Kontext ein fester allgemeiner Ordnungsrahmen und (gewöhnlich) eine "krönende" Justizinstanz fehlt, ist die Praxis in besonderem Masse auf Unterstützung durch die Rechtswissenschaft angewiesen. Die Wissenschaft sollte ihre Aufmerksamkeit nicht nur auf Rechtsschutz- und Legitimationsfragen richten, sondern auch bei der Suche nach 
"funktionalen Äquivalenten“ (Ausgleich struktureller Defizite) eine aktive Rolle übernehmen.

21. Im „ebenenübergreifenden“ Vollzug müssen mitunter starke Interessengegensätze verarbeitet werden. Bei der Analyse interessiert nicht zuletzt auch ein allfäliger "Interessengleichklang" (Stichwort: Aktivierung Privater). Erkenntnisse der Gesetzgebungslehre sind vermehrt für die „Internationale Rechtsetzung" (inkl. Sekundärrecht) fruchtbar zu machen. Ein zentrales Anliegen ist die systematische Prüfung der Vollzugstauglichkeit von geplanten Regelungen.

22. Das nationale Verwaltungsrecht ist auf exportfähige Gedanken und Rechtsfiguren hin zu untersuchen. Gefragt ist nicht nur ein horizontaler Ländervergleich, sondern auch ein vertikaler Vergleich der verschiedenen Varianten dezentralen Vollzugs (Bundesstaat, EU, Völkerrecht).

\section{Zur Einordnung des „Internationalen Verwaltungsrechts“}

23. Die Entwicklung eines "Systems des Internationalen Verwaltungsrechis" ist kein vorrangiges Anliegen.

24. Zentraler Gegenstand eines "Internationalen Verwaltungsrechts" sind jene Rechtsfiguren und Strukturen, welche die Rechtsverwirklichung organisieren - mit Blick auf das Ziel eines vorgabengetreuen, gleichmässigen (und auch im Übrigen rechtsstaatlich und demokratisch einwandfreien) Verwaltungsvollzugs. Aus der Sicht der Rechtsquellen handelt das "Internationale Verwaltungsrecht" von Regelungen, die teils dem Völkerrecht, teils dem nationalen (oder europäischen) Recht zuzuordnen sind. Eine Aufspaltung in einen nationalen und einen internationalen Teil sollte vermieden werden.

25. Das „Internationale Verwaltungsrecht “ ist nicht als ein primär am positiven Recht orientiertes Rechtsgebiet zu konzipieren, sondern als eine in erster Linie an theoretischen Grundlagen und Grundfragen interessierte Disziplin (im Sinne von "Allgemeinen Lehren").

26. Inhaltlich und strukturell besteht eine grosse Nähe zum sog. Allgemeinen Verwaltungsrecht. Es empfiehlt sich, das "Internationale Verwaltungsrecht" (verstanden als auf die internationale Dimension bezogene Verwaltungsrechtstheorie) in die Allgemeinen Lehren des rechtsstaatlichdemokratischen Verwaltungsrechts zu integrieren.

27. Die sich abzeichnende Herausbildung eines "Internationalen Verwaltungsrechts" sollte zum Anlass genommen werden, der Verwaltungsrechtstheorie und der Verwaltungsrechtsvergleichung generell noch mehr Aufmerksamkeit zu schenken. 


\section{Aussprache und Schlussworte}

\section{Die Entwicklung eines Internationalen Verwaltungsrechts als Aufgabe der Rechtswissenschaft}

Ress: Ich möchte den beiden Referenten zu ihren hervorragenden Referaten gratulieren. Das war wirklich eine glanzvolle Leistung auf dem Gebiet der Mischung zwischen Völkerrecht, Europarecht und nationalem Recht, auf einem Gebiet, das schwer zu fassen ist. Damit komme ich gleich zur Frage des Begriffs und der Kategorisierung. Ich bin froh, dass Sie zum Teil Abschied genommen haben von dem Begriff des internationalen Verwaltungsrechts als eines kollisionsrechtlichen Begriffs und sich auf die Suche gemacht haben nach einem neuen Begriff. Also einem Begriff, der in der Nähe eines europarechtlichen oder völkerrechtlichen Mischbegriffs steht. Ich habe mich gewundert, Herr Biaggini, dass Sie zum Schluss nicht den Mut gehabt haben zu sagen, es ist eine neue Kategorie, die wir hier aus einer Fülle von Indizien kreieren und zu der wir uns bekennen. Wir haben in Deutschland, aber auch in Europa eine ganze Reihe von Beispielen. Es ist erwähnt worden dieses saarländische Grenzabkommen mit einer Schiedsinstanz. Das ist natürlich eine Lösung eines solchen Regimes. Wir haben mit der Rheinschifffahrtskommission ein separates Rheinregime, das total anders durchorganisiert ist als unsere deutsche Rechtsordnung: mit einem eigenen Umweltrecht, mit einem eigenen Verwaltungsrecht, mit einem eigenen Gericht in Straßburg. Ich weiß, es ist schwierig, dieses Rechtsgebiet als eigene "Sonderrechtsordnung“ zu qualifizieren, wenn man keinen Vertrag als Grundlage hat, den man völkerrechtlich einordnen könnte, aber wir sollten uns auf die Suche machen nach einer eigenen Kategorie des internationalen Verwaltungsrechts. Ich weiß, dass es schwierig ist, ohne einen solchen Vertrag diese Fragen anzugehen. Sehr beeindruckt hat mich auch das Kieler Hafenabkommen. Auch das ist ein grenzüberschreitendes internationales Verwaltungsregime. Was mich auch beeindruckt hat, war die These 17 von Ihnen, Herr Biaggini, mit der Organisation einer kollektiven Aufsicht. Das halte ich in der Tat für die künftige Entwicklung furr eine sehr leistungsfähige Idee. Wir werden vielleicht nicht so schnell zur Gründung von gerichtlichen Instanzen im Zusammenhang mit dem internationalen Verwaltungsrecht kommen, aber das so zu organisieren, dass es hier eine kollektive grenzüberschreitende internationale Aufsicht gibt, halte ich doch für einen interessanten Ansatz. 
von Bogdandy: Auch ich möchte den Referenten zu ertragreichen Vorträgen gratulieren. Erlauben Sie mir eine Nachfrage zum Begriff des internationalen Verwaltungsrechts und zu den Gründen seines schnellen Erfolgs. Der Begriff ,internationales Verwaltungsrecht“ kommt heute im Wesentlichen aus den USA und aus Italien zu uns. Warum hatte er dort einen großen Erfolg? Insbesondere in den USA hat er großen Erfolg, weil dieser Begriff ein „Rettungsbegriff“ ist, mit dem die Internationalisten in Amerika im Kontext erheblicher Ablehnung des Völkerrechts gewisse Teile aus der "Schusslinie“ nehmen wollen. Dieses Problem haben wir nicht: Deutschland und die Schweiz sind prinzipiell völkerrechtsfreundliche Staaten, auch die Verfassungsrechtler sind prinzipiell völkerrechtsfreundlich. Dann haben wir den von Italien aus geprägten Diskurs. Was ist da der Hintergrund? In Italien gilt die strikte Trennung von Verwaltungsrecht, Verfassungsrecht und Völkerrecht. Höchst sensibel wird reagiert, wenn der eine in das andere Feld hineingeht. Das diritto amministrativo globale kann als Versuch des Verwaltungsrechts gedeutet werden, sich gewisser Bereiche des Völkerrechts zu bemächtigen; sozusagen als disziplinäre Landnahme. Auch dieses Problem haben wir nicht, weil in Deutschland unter dem Dach der Staatsrechtslehrervereinigung Völkerrechtler, Staatsrechtler und Verwaltungsrechtler in größter Freundschaft zusammenleben. Warum also der Erfolg in Deutschland? Der Begriff des internationalen Verwaltungsrechts erlaubt es, das Programm der Konstitutionalisierung des Völkerrechts, also einer Verrechtlichung der Welt, das uns schon in Hamburg beschäftigt hat, weiterzuführen. Der Begriff des internationalen Verwaltungsrechts kommt zudem auf einer ruhigeren Ebene daher und erspart diesem Projekt, an den Klippen des Verfassungsbegriffs zu zerschellen. Allerdings besteht weiterhin die große Gefahr, dass der Gedanke zu schnell in protofóderale Kategorien abrutscht, dass zu schnell in den Kategorien der Europäischen Union gedacht wird. Wir müssen aufpassen, dass wir nicht Opfer unserer hohen Abstraktionsleistung werden, und dass wir die Felder trennen: zum Einen dieses internationale Verwaltungsrecht als eine Entwicklung des Rechts der internationalen Organisationen, zum Anderen ein internationalisiertes internes Verwaltungsrecht als ein international offenes nationales Verwaltungsrecht. Diese Trennung der beiden Materien ist wichtig, um dem Thema in diesem Stadium seiner Entwicklung gerecht zu werden.

Tietje: Ich möchte zunächst nicht nur den Referenten für sehr anregende Vorträge danken, sondern ebenso dem Vorstand für die Wahl des heutigen Themas. Die Fragen nach Existenz und Strukturen eines - wie es hier genannt wurde - internationalen Verwaltungsrechts, und hier 
möchte ich Herrn von Bogdandy widersprechen, kommt genuin aus der deutschen bzw. europäischen Rechtswissenschaft. Zu nennen sind insofern beispielhaft nur die Namen Robert von Mohl und später dann in unserer Vereinigung Hartwig Bülck, Eberhard Menzel und Jost Delbrück. Liest man die genannten und andere Autoren, wird schnell klar, dass Vieles von dem schon analysiert und gedacht wurde, was heute unter Stichworten wie "global administrative law" als vermeintlich ganz neue Erfindung gilt.

Damit komme ich zugleich zur Problematik, ob wir wirklich von ,internationalem Verwaltungsrecht" sprechen sollten. Mir erscheint dieser Begriff doch im Vergleich zu anderen Teildisziplinen der Rechtswissenschaften zu sehr durch das Rechtsanwendung- und Kollisionsrecht belegt. Insofern besteht die Gefahr, dass mit einem methodischen Ansatz, der - wie die heutigen Referate gezeigt haben - die kollisionsrechtliche Perspektive gerade nicht behandelt, die wichtigen Fragen eines Rechtsanwendungs- und Kollisionsrechts im Öffentlichen Recht insgesamt nicht mehr behandelt werden. Dass die Referenten auf diese Thematik nicht eingegangen sind, hat gute Gründe und soll hier nicht kritisiert werden. Wichtig scheint mir dessen ungeachtet jedoch zu sein, dass es um eine möglichst umfassende Analyse von Internationalisierungsphänomen im öffentlichen Recht gehen muss. Insofern geht es bei der verstärkten Einbindung der Verwaltung in internationale Regelungs- und Regulierungsstrukturen um Rechtsanwendungs- und kollisionsrechtliche Fragen ebenso wie um Analysen zur strukturellen Internationalisierung der Verwaltung. Damit muss ein Begriff gefunden werden, der die Gesamtmaterie der Internationalisierung der Verwaltung abdeckt.

$\mathrm{Zu}$ einer weiteren Anmerkung und Frage an die Referenten möchte ich ein Stichwort erwähnen, das in den Referaten nicht vorkam. Konkret geht es um das Territorialitätsprinzip, das das deutsche Verwaltungsrecht in historischer Perspektive bekanntlich zentral geprägt hat. Nun hat das Territorialitätsprinzip durch das europäische Verwaltungsrecht zwar schon deutliche Modifikationen erfahren, als Grundannahme des wissenschaftlichen Verständnisses vom deutschen Verwaltungsrecht scheint es mir aber weiterhin sehr präsent zu sein. Es stellt sich damit die Frage an die Referenten, ob das Territorialitätsprinzip vor dem Hintergrund der überzeugenden heutigen Referate überhaupt noch rechtliche Bedeutung hat oder ob es nicht Zeit ist, endgültig hiervon Abschied zu nehmen. Wenn das so sein sollte, ist in einem nächsten Schritt zwangsläufig zu fragen, welche Auswirkungen dies auf Grundstrukturen des internationalen Verwaltungsrechts in seiner rechtsanwendungs- bzw. kollisionsrechtlichen Perspektive hat. Das führt dann übrigens auch zur Frage nach überholten rechtlichen Ansätzen im Sinne eines Dualismus 
von innerstaatlichem und internationalem Recht, worauf Herr Classen überzeugend hingewiesen hat. Das Bundesverfassungsgericht geht in seiner jüngeren Rechtsprechung hingegen einen ganz anderen Weg und versucht - so mein Eindruck - eine Rückkehr zu Denkstrukturen eines radikalen Dualismus durchzusetzen.

Röhl: Ich will mich mit dem Begriff des Internationalen Verwaltungsrechts im Hinblick auf die davon erfassten Gegenstände beschäftigen. Das wird dann interessant, wenn man sich die kleine entwicklungsgeschichtliche Hypothese zur Konjunktur dieses Themas ansieht, die ich anzubieten habe. Diese Konjunktur könnte ja daraus resultieren, dass man dem letzten großen Thema, dem europäischen Verwaltungsrecht, keinen Neuigkeitswert mehr zumisst und jetzt darüber hinausgreifen bzw. das europäische Recht hinter sich lassen möchte. Hier lohnt es sich aber, darauf hinzuweisen, dass das Europarecht durch die Hintertür wieder hereinkommt: Denn ein Großteil der Gegenstände, die wir als internationale verwaltungsrechtliche Phänomene beschreiben, kommen gar nicht durch den direkten Kontakt zwischen nationalem Recht und Völkerrecht zustande, vielmehr werden die völkerrechtlichen Einwirkungen über Europarecht vermittelt, und zwar über völkerrechtliche Bindungen der Europäischen Gemeinschaft. Ich nenne Beispiele, die gestern und heute angeführt wurden, WTO, Umweltabkommen, Aarhus-Konvention, Rechnungslegungsstandards, Basel II, alle diese Gegenstände wirken vermittelt über das Europäische Recht auf das nationale Recht ein. Müsste man daher nicht, wenn man nach Rechtsprinzipien fragt, wie es gestern geschehen ist oder wie es Herr Classen heute anhand des Demokratie- und Rechtsstaatprinzips getan hat, versuchen, dem einen zweiten Teil zur Seite zu stellen? Dieser hätte dann den Zugriff auf die Fragen des internationalen Verwaltungsrechts aus der Perspektive des Europäischen Verfassungsrechts zu konstruieren, indem er nach europarechtlichen Verankerungen und Konsequenzen der soeben genannten Prinzipien fragt.

Mayer: Ich kann in gewissem Sinne an das anknüpfen, was bereits gesagt worden ist. Ich meine die Beobachtung, dass die internationale Dimension sich in vielerlei Hinsicht bei genauerem Hinsehen im Kern als europäisches Phänomen entpuppt und Verbindungslinien zwischen Europäisierung und Internationalisierung bestehen. Vor diesem Hintergrund meine Frage: Wir haben ein Panorama von vielfältigen und interessanten Entwicklungen präsentiert bekommen, die die internationale Dimension von Verwaltungsrecht betreffen - aber gibt es auch Risiken und Gefahren dieser Entwicklung? Ich komme zu dieser Frage durch 
die Befassung mit der Internationalisierung des nationalen Verwaltungsrechts, und zwar insbesondere dem, was man die Einwirkungsdimension nennen könnte. Wer sich damit auseinandersetzt, kann sich gut an das erinnern, was gewissermaßen die Vorläuferfragestellung zur Internationalisierungsfrage war, nämlich die Frage nach der Europäisierung des nationalen Verwaltungsrechts. Diese hat heftige Abwehrreaktionen hervorgerufen und ist von der deutschen Verwaltungsrechtswissenschaft mit zum Teil sehr skeptischen Bemerkungen begleitet worden, bis hin zu einer spürbaren Abwehrhaltung. Ihnen ist vielleicht noch dieses Bild in Erinnerung vom festgefügten Schiff des deutschen Verwaltungsrechts, das bald zu kentern drohe wegen des eindringenden europäischen Rechts. Von vergleichbaren Risiken und Gefahren haben wir wenig gehört heute. Schon alleine weil dies nicht thematisiert worden ist also meine Nachfrage, ob die Internationalisierung des Verwaltungsrechts nicht doch Gefahren und Risiken der Natur aufweist, wie sie seinerzeit für das Phänomen der Europäisierung zu recht oder zu unrecht gesehen worden sind - oder besteht allenfalls die Gefahr dass die Völkerrechtler neben sich plötzlich die Verwaltungsrechtler auftauchen sehen, die an ihren Gegenständen forschen wollen?

Oppermann: Zunächst einmal herzlichen Dank den beiden Referenten. Es war bereichernd, Ihnen zuzuhören, Herr Classen. Sie haben zwar nicht ganz die ICE-Geschwindigkeit von Herrn Appel heute Morgen erreicht, aber wer Ihnen zu folgen vermochte, wurde belohnt.

Eine Bemerkung zur vertikalen Internationalisierung der Verwaltung zwischen den internationalen Institutionen und dem nationalen Bereich. Die Verwaltung ist inzwischen im Sinne des schönen Begriffes des dédoublement fonctionnel von Georges Scelle, den Sie gebracht haben, Herr Biaggini, zwei Ebenen verpflichtet. Das zu erkennen, ist wichtig.

Wir sollten in diesem Zusammenhang einen Blick auf die Realiät der supra- oder internationalen Verwaltung werfen. Sie ist heute zu einem ausgesprochen multinationalen Amalgam geworden. Diese Verwaltung besteht mittlerweile in der EU aus 27 Nationalitäten. Von der UNO oder der WTO mit ihren 190 oder 150 Mitgliedstaten spreche ich nicht einmal. Diese multinationalen Administrationen wirken auf die nationalen Rechts- und Verwaltungskulturen ein. Wenn ich die EU nehme, administriert die supranationale Kommissionsverwaltung ausgesprochen national geprägte Bereiche wie Wettbewerb, Daseinsvorsorge, den weiten Bereich der Rechtsangleichung und vieles Andere. Mittels der PISAUntersuchungen und Empfehlungen der OECD-Verwaltung belehrt uns ein mexikanischer Delegationsleiter über die Richtigkeiten oder Unrichtigkeiten des deutschen Bildungssystems. 
Da liegt ein Problem. Herr Biaggini, Sie haben mit Recht von dem notwendigen Vertrauen der nationalen Rechtsunterworfenen in die übernationale oder internationale Rechtsordnung und in ihre Verwaltungsentscheidungen gesprochen. Daran schließt sich meine Frage an die beiden Referenten an. Auf welchen Grundlagen beruht diese internationale Verwaltung? Man kann sich grundsätzlich zwei Varianten vorstellen. Einerseits mag es bestimmte nationale „Leitkulturen“ der Verwaltung geben, wenn ich das mal so sagen darf, aus denen die übernationale Verwaltung ihre Maßstäbe bezieht. Sie haben ähnlich von ,allgemeinen Lehren“ gesprochen, Herr Biaggini. Wenn man an die EU denkt, ist das europäische Beamtenstatut wesentlich aus deutsch-französischen Wurzeln gewachsen. Es gibt da starke deutsche Einschläge, wie Unkündbarkeit, Lebenszeitprinzip. Ablehnung einer Rotation. Die europäische Gerichtsverfassung ist ihrerseits in den fünfziger Jahren aus deutsch-französischen Modellen entstanden. Aber wie ist es bei den Vereinten Nationen mit ihren 190 Mitgliedern? Ich weiß das nicht. Auch dort muß es irgendwelche überzeugende Lehren geben, Begrifflichkeiten, Prinzipien, die zur konkreten Gestaltung dieses internationalen Verwaltungsrechts geführt haben. Darüber würde ich gerne ein bisschen hören.

Peters: Vielen Dank für die sehr schönen Referate. Ich habe drei Fragen an beide Referenten. Die erste Frage betrifft das von Giovanni Biaggini genannte Stichwort der Aktivierung Privater für die Durchsetzung der internationalen Vorgaben im Rahmen der vertikalen Internationalisierung. Hier hatte Herr Biaggini gefordert, die nationalen Gerichte sollten die unmittelbare Anwendbarkeit der völkerrechtlichen Vorgaben ruhig großzügiger bejahen. Im Gegensatz dazu hat sich Herr Classen jedenfalls in Bezug auf das Sekundärrecht internationaler Organisationen zurückhaltend geäußert und sinngemäß verlangt, dass dieses nicht ohne weiteres unmittelbar anwendbar sein solle. Meine Frage ist, welche Schwierigkeiten hier gesehen werden. Die völkerrechtlichen Vorgaben sind einerseits sehr oft von ihrem Wortlaut her ohnehin ungeeignet zur unmittelbaren Anwendbarkeit. Andererseits ist umstritten, ob die Frage der unmittelbaren Anwendbarkeit einer völkerrechtlichen Norm überhaupt eine nationalrechtliche ist. Anders gewendet: Dürfen die nationalen Gerichte diese nach eigenen Kriterien beantworten oder müssen nicht vielmehr internationale Vorgaben auch für die Beurteilung der unmittelbaren Anwendbarkeit einer internationalen Norm beachtet werden? Ich neige der letzteren Auffassung zu, weil ich die Zuerkennung der unmittelbaren Anwendbarkeit als einen Aspekt der Vertrags(oder Sekundärakts-)interpretation ansehe, die grundsätzlich den völkerrechtlichen Grundsätzen zu folgen hat. 
Meine zweite Frage ist, wie Sie die Einbindung privater Akteure in das internationale Verwaltungsrecht im engeren Sinne bewältigen. Diese spielen meiner Ansicht nach eine sehr große Rolle, angefangen von Wirtschaftsverbänden bis zu ganzen Branchen oder Gewerkschaften. Müsste man hier nicht eine Art internationales Privatisierungsfolgenrecht mit konstruieren?

Meine dritte Frage stelle ich im Anschluss an die Bemerkung von Herrn von Bogdandy. Sehen Sie auch in der Idee des global administrative law (GAL) die Fortsetzung der Konstitutionalisierung des Völkerrechts mit anderen Mitteln oder handelt es sich hier um ein Gegenkonzept? Mir scheint das Selbstverständnis der Vertreter des GAL zu sein, dass sie ein - im Gegensatz zum sehr wertaufgeladenen Konstitutionalisierungsbegriff - neutrales und technisches global administrative law propagieren. Ich halte dieses Selbstverständnis für irreführend. Im Grunde genommen geht es um dieselben Fragen, nämlich um Rechenschaftspflichtigkeit, Transparenz, Anhörungsrechte und so weiter. Sowohl die Rekonstruktion von Teilen des internationalen Rechts als Verwaltungsrecht als auch die Verfassungslesart verfolgen das Anliegen der verbesserten „rule of law" auf internationaler Ebene.

Schönberger: Ich wollte eine Anmerkung machen zu dem, was Herr Biaggini gesagt hat. Ich finde es sehr schön, dass Herr Biaggini eine alte wissenschaftliche Form wieder aufgegriffen hat, dass er nämlich die Verbindung von Bundesstaatsrecht und Völkerrecht neu belebt. Es geht dabei darum, Erfahrungen aus dem Bundesstaatsrecht fruchtbar zu machen für das Verständnis neuer völkerrechtlicher Probleme. Wenn wir genau hinsehen, ist das in der Vergangenheit auch immer wieder so gemacht worden. Ich erinnere für Deutschland nur etwa an Heinrich Triepel, der völkerrechtliche und bundesstaatsrechtliche Forschung miteinander verbunden hat. In seiner großen Studie über die Reichsaufsicht (1917) hat er gerade die Phänomene, die Herr Biaggini hier für das Europa- und Völkerrecht untersucht, für das Bundesstaatsrecht einmal durchsystematisiert. Das Verständnis der allgemeinen Probleme der Durchsetzbarkeit und Vollzugskontrolle für das Europarecht und das Völkerrecht gewinnt an Klarheit, wenn es sich die bundesstaatsrechtliche Forschung zunutze macht. Dabei ist nun noch ein zweiter Gesichtspunkt von Bedeutung. Herr Biaggini hat darauf hingewiesen, dass er entsprechende Parallelerfahrungen häufig in der Geschichte findet, etwa im frühen Schweizer Bundesstaat im 19. Jahrhundert. Als die Verhältnisse in den Bundesstaaten noch ungesichert waren, haben sich die Probleme der Durchsetzung des Bundesrechts (etwa die Rebellion von Obergerichten der Gliedstaaten etc.) in besonderer Deutlichkeit gezeigt. 
Wir können die entsprechenden Phänomene des Europa- bzw. Völkerrechts gerade durch eine verfassungsgeschichtliche Betrachtung der Bundesstaaten besser begreifen. Es geht also zum einen um eine erneuerte Verbindung von Bundesstaatsrecht und Völkerrecht, zum anderen darum, heutige Phänomene des dezentralen Vollzugs übernationalen Rechts durch die Parallelisierung mit früheren Phasen in der Entwicklung der Bundesstaaten besser zu verstehen.

Pitschas: Ich erlaube mir an beide Referenten, verbunden mit bestem Dank für die Referate, drei kleinere Nachfragen. Diese nehmen ihren Ausgangspunkt bei der begrifflichen Klärung dessen, was internationales Verwaltungsrecht bedeutet. Mich interessiert daran natürlich die internationale Seite, aber auch das Verständnis von Verwaltungsrecht. Dieses wendet sich einerseits an die Verwaltung. Wenn Sie so wollen, ist es dimensionales Steuerungsrecht. Verwaltungsrecht wendet sich andererseits an die Bürger, also an diejenigen, die mit der Verwaltung in Kontakt treten. Sie ahnen schon, worauf meine erste Frage zielt, nämlich auf die Verbindung nicht zu "Privaten“, wie Herr Biaggini in These 21 und soeben Frau Peters dargelegt haben, sonder auf die Verbindung zu „Bürgern“; gefragt wird somit nach der Legitimation und legitimen Entwicklung des internationalen Verwaltungsrechts. Es gibt in einem Bereich des sich ausprägenden internationalen Verwaltungsrechts ganz besonders Grund zu dieser Nachfrage, das sind im Welthandelsrecht die Aktivitäten der WTO. Wenn man sich diese ansieht, so muss man feststellen, dass hier vor allem die verwaltungsrechtlichen Strategien der beteiligten Staaten einerseits in Konflikt mit der defizitären Struktur ihrer Rückbindung an Bürger oder Bürgergruppen andererseits geraten. Eine Antwort auf die Frage nach der demokratischen Legitimation von internationalem Verwaltungsrecht finden wir aber, Herr Classen nur in einem von Ihnen nicht erwähnten partizipativen Ansatz.

Die zweite Frage, die sich mir aufdrängt, wendet sich an Herrn Biaggini. Sie haben das Wirtschaftsverwaltungsrecht als Teil des internationalen Wirtschaftsrechts erwähnt. Müsste man nicht überhaupt angesichts solcher gebietsbezogener Verselbständigungstendenzen sektorale Erklärungsansätze bevorzugen? So wäre z.B. an ein Entwicklungsverwaltungsrecht zu denken, das in besonderer Weise die strukturellen Defizite der Legitimation rechtlicher Ordnung der Beziehungen zu Entwicklungsländern aufzunehmen hätte. Ich darf bei Herrn Biaggini noch bleiben und die These 17, die Herr Ress schon angesprochen hat, noch einmal aufgreifen. Ich bin skeptisch, Herr Biaggini, ob wir gerade mit dem Institut der Aufsicht ein allgemeines internationales Verwaltungs- 
recht begründen helfen könnten. Wenn Sie etwa im EU-Bereich sehen, wie eine europäische Finanzdienstleistungsaufsicht ganz informell im Rahmen der EZB entsteht, aber formell-rechtlich noch kein weiterer Schritt zu ihrer Institutionalisierung getan wurde, so unterstreicht das meine Skepsis.

Kotzur: Beide Referate haben nach einem Ordnungsrahmen für das internationale Verwaltungsrecht gesucht und damit ihr Vorverständnis geoffenbart. Internationales Verwaltungsrecht ist mehr als Kollisionsrecht, denn Kollisionsrecht hätte in den nationalen Verfassungen und dem daraus abgeleiteten „ordre public“ einen hinreichenden Ordnungsrahmen. Das Völkerrecht als „konstitutionelle“ Ordnung kann einen vollständigen Ordnungsrahmen noch nicht liefern, und selbst wer die völkerrechtliche Konstitutionalisierungsdebatte optimistisch und idealistisch verfolgt, wird nicht so vermessen sein, die völkerrechtliche Ordnung mit einer nationalen Vollverfassung gleichsetzen zu wollen. Ich darf deshalb an Frau Peters und an Herrn von Bogdandy anknüpfen. Ich glaube in der Tat, dass die verwaltungsrechtliche Debatte auf internationaler Ebene einer Konstitutionalisierung von unten nach oben auf die Beine helfen kann, und dass bei dieser Konstitutionalisierungsidee die vorhandenen Verwaltungsstrukturen eine wichtige Rolle spielen. Und so wie Konrad Hesses offener Verfassungsbegriff - das sollte hier in Freiburg einmal nachdrücklich betont werden - der völkerrechtlichen Konstitutionalisierungsdebatte fruchtbare Ansätze bieten kann, kann vielleicht auch die Ordnungsidee von Herrn Schmidt-Aßmann bei der völkerrechtlichen Konstitutionalisierung als Systembildung fruchtbar gemacht werden. Drei Beispiele: Eine internationale "rule of law" lässt sich gewiss nicht nur aus irgendwelchen vorgegebenen obersten Prämissen axiomatisch deduzieren, sie muss vielmehr durch die „rechtsstaatlichen" Strukturen, die internationale Verwaltungsregime kennen, Bestätigung finden. Ein zweites Beispiel: Die Frage nach der Rechtswahl, nach der Anknüpfung an die relevante Rechtsordnung wird wichtiger denn je, weil die dirigierende Rechtsordnung entscheidet, wie dicht gewirkt deren Schutzstrukturen, gerichtlicher Rechtsschutz, demokratische Legitimation etc. sind. Eine wichtige Rationalitäts- und Systembildungsfunktion wird sein, nach den Rechtswahl- bzw. Anknüpfungskriterien zu fragen. Und ein drittes Beispiel: In nationalen Verfassungen gibt es eventuell nach dem Vorbild von Art. 24 Abs. 1a GG manche Öffnungsklauseln, die von der Praxis noch nicht, jedenfalls noch nicht hinreichend genutzt werden. Hier könnte das internationale Verwaltungsrecht ermutigen. 
Sydow: Herr Biaggini hat - in Aufnahme des Gesamttagungsthemas nach der Aufgabe der Rechtswissenschaft im Hinblick auf das Internationale Verwaltungsrecht gefragt und als eine der Antworten die Suche nach exportfähigen Rechtsfiguren der nationalen Rechtsordnungen benannt. Auch Herr Röhl hat dieses Thema angesprochen, als er über die Transformation von Rechtsprinzipien über das Europarecht sprach. Ich sehe die Attraktivität derartiger Export- und Vermittlungsversuche für die Aufgabe, ein Internationales Verwaltungsrecht zu entwickeln. Denn diese Aufgabe wird umso mehr erleichtert, je weniger Wertungswidersprüche es zwischen den einzelnen Rechtsordnungen zu überbrücken gilt. Ich möchte aber gleichwohl vor allzu großen Hoffnungen in dieser Hinsicht warnen. Die Aufgabe, ein internationales Verwaltungsrecht zu entwickeln, wird auf Dauer mit dem Problem konfrontiert bleiben, dass die beteiligten Verwaltungsrechtsordnungen in vielen Wertungsfragen grundsätzlich verschieden sind. Diese Skepsis gegenüber einer Konvergenzhoffnung durch Transport und Rezeption dogmatischer Figuren hat drei Gründe. Es gibt - erstens - zwar durchaus viele Entwicklungen, die eine Konvergenzthese stützen: In Frankreich diskutiert man über Vertrauensschutzgesichtspunkte; in Großbritannien gibt es so etwas wie einen Verhältnismäßigkeitsgrundsatz; in Deutschland - ich erinnere an den heutigen Vormittag - finden Verfahrensgedanken verstärkte Berücksichtigung. Aber es gibt ebenso gut Gegenbeispiele einer Auseinanderentwicklung: So ist etwa die Ausbildung einer rein gerichtszentrierten Konzeption des Verwaltungsrechtsschutzes durch weitreichende $\mathrm{Ab}$ schaffung des Widerspruchsverfahrens der VwGO ohne jede Parallelentwicklung in anderen europäischen Rechtsordnungen. Von einer generellen Konvergenz nationaler Verwaltungsrechtsordnungen kann deshalb keine Rede sein. Zweitens: Soweit es - unbestritten - Konvergenzentwicklungen gibt, so ist zu bedenken, dass sich eine Rechtsfigur bei ihrer Rezeption durch eine andere Rechtsordnung wandelt. Sie ist nicht mehr dieselbe wie in der Ausgangsrechtsordnung. Was in Großbritannien proportionality genannt wird, ist nicht das, was wir als Verhältnismäßigkeitsgrundsatz kennen. Und drittens: So wünschenswert Konvergenzentwicklungen sind und so sehr man sie in verschiedenen Bereichen konstatieren kann, so wenig lassen sie sich durch gezielte Exportanstrengungen oder die Suche nach exportfähigen Rechtsfiguren befördern. Das lässt sich beispielsweise für verschiedene Annäherungen des britischen öffentlichen Rechts an kontinentaleuropäische Vorstellungen zeigen, etwa für verstärkte Verfassungsbindungen des politischen Prozesses oder für intensivierte Gerichtskontrollen exekutiven Handelns. Wenn man diese Entwicklungen überhaupt als Europäisierung bezeichnen mag, so sind sie jedenfalls nicht Folge europäischer Einwirkun- 
gen. Sie sind vielmehr in der britischen Verfassungstradition selbst angelegt und aus ihr heraus entwickelt, so dass Europäisierungseinflüsse hier nur als Katalysatoren wirken können.

Kugelmann: Herr Classen, Sie haben mit großer Präzision die Bereiche identifiziert, in denen das internationale Verwaltungsrecht eine Rolle spielt. Einer davon ist die Informationssammlung und -auswertung und Sie haben zu Recht auf den sich schnell entwickelnden Bereich des internationalen Polizei- und Sicherheitsrechts hingewiesen. Daran möchte ich anknüpfen und die Frage stellen, ob wir eine spezifische Informationsordnung für den Informationsaustausch im grenzüberschreitenden Bereich brauchen. Und zwar weit über die bisher existierenden, aber stark zersplitterten Regelungen im BKA-Gesetz, im Verfassungsschutzgesetz usw. Denn auf diesem Gebiet fehlt doch die Transparenz, gerade auch für die Betroffenen hinsichtlich der Daten, die möglicherweise ausgetauscht oder weitergegeben werden. Dies betrifft vorrangig die unterschiedlichen Polizeibehörden und erst recht die Sicherheits- oder Nachrichtendienste, wobei insoweit die Herstellung von Transparenz etwas schwierig ist. Konkreter formuliert: Gebieten die Schutzpflichten aus den Grundrechten, zumal im Rahmen des Datenschutzes, dass verstärkt Regelungen in der innerstaatlichen Rechtsordnung getroffen werden müssen, um eine Informationsordnung, einen Informationsaustausch sicherzustellen, der dem Datenschutz ebenso wie der Informationsfreiheit Rechnung trägt? Das wiederum würde bedeuten, damit wende ich mich an Herrn Biaggini, dass wir eine bereichsspezifische Regelung internationalen Verwaltungsrechts für das Sicherheitsrecht bräuchten, denn diese Anforderungen haben wir in anderen Bereichen so nicht. Das würde ein wenig Ihrer These widersprechen, dass insbesondere allgemeine verwaltungsrechtliche Fragen hier eine Rolle spielen, also der Einordnung des internationalen Verwaltungsrechts in den Kontext des Allgemeinen Verwaltungsrechts. Deshalb stellt sich die Frage, ob wir nicht zugleich die spezifischen Besonderheiten stets mit bedenken müssen, um zu wissen, was wir im Allgemeinen regeln können und in welchen Bereichen wir vielleicht gerade besondere spezifische Regelungen brauchen. Mit einem letzten Punkt wende ich mich auch an Sie, Herr Biaggini, und kann insoweit an Herrn Sydow anschließen. Ich möchte ein wenig zur Vorsicht mahnen bei der Übertragung von Kriterien aus dem EU-Recht oder konkreter aus dem EG-Recht in das internationale Verwaltungsrecht. Dies knüpft an Erfahrungen etwa mit Fragen an, die sich bei der Cyber Crime-Konvention des Europarates und dem entsprechenden Rahmenbeschluss der EU stellen, also hinsichtlich der Bekämpfung der Computerkriminalität über die Grenzen hinweg. Vieles 
was in der Europäischen Union funktioniert, funktioniert deshalb, weil wir uns gegenseitig vertrauen, weil es eine gemeinsame Wertegrundlage und einen gemeinsamen Ordnungsrahmen gibt. Wir sind also weniger zögerlich, den Polizei- und Sicherheitsbehörden in Frankreich oder auch der Schweiz, auch wenn sie nicht Mitglied ist, Daten zu überlassen, während die Bedenken etwas größer sind, wenn die Anfrage aus Aserbaidschan oder Georgien kommt. Diese Grundlage des gemeinsamen Ordnungsrahmens muss auf jeden Fall mitbedacht werden, wenn Sie denn für ein allgemeines, und gerade auf Drittstaaten bezogenes internationales Verwaltungsrecht Grundlagen suchen.

Raschauer: Was den internationalen Datenschutz betrifft, wäre ich skeptischer: Wenn wir die amerikanischen Safe harbour-Regelungen lesen, so haben diese nichts mit europäischem Datenschutz gemeinsam. Datenschutz, das war ohnedies mein erster Punkt. Ich meine, dass der Begriff des internationalen Verwaltungsrechts als Kollisionsrecht vielleicht doch noch auf Lager gehalten werden muss. Die Datenschutzrichtlinie der EG hat eine Bestimmung über den räumlichen Anwendungsbereich. Daraus ergibt sich, dass die österreichische Datenschutzkommission dann deutsches Recht anzuwenden hat, wenn der Server in Deutschland steht und intentional auf eine Datenverarbeitung in Österreich ausgerichtet ist. Bei Neumeyer lesen wir noch, dass internationales Verwaltungsrecht dadurch gekennzeichnet ist, dass es keine zweiseitigen Kollisionsnormen hat. Das gebe es nur im privatrechtlichen IPR. Da sollten wir nicht das Kind mit dem Bad ausschütten, indem wir die internationalen Prozesse in den Vordergrund stellen. Es gibt spezifisch kollisionsrechtliche Fragen im Verwaltungsrecht. Ansonsten wollte ich gewissermaßen als eine Minute österreichischer Landesbeitrag - ergänzend darauf hinweisen, dass nach meinen Wahrnehmungen das derzeit aktuellste Thema in Österreich die internationale Amtshilfe ist. In diesem Zusammenhang ist die internationale Vorortkontrolle in allen Bereichen der Finanzdienstleistungsaufsicht das Spannendste. Das gemeinschaftsrechtliche Regime sieht vor, dass die nationale Herkunftsstaatsbehörde ihre Schäfchen durch ganz Europa begleitet und dort Akteneinsicht in Brüssel, in London oder wo immer Finanzplätze sind, nimmt. Das ist aber - ich bin dankbar für die Worte - Netzwerke-, Vertrauen- und Verwaltungskulturen-abhängig. Wenn man mit den Mitarbeitern der österreichischen FMA spricht, dann gibt es Fälle, da lädt man bei einer grenzüberschreitenden Bewilligung, bei qualifizierten Beteiligungen oder Verschmelzungen, die beteiligten Behördenvertreter nach Wien zu einer Schale Kaffee, und nachher kommen in den beteiligten Staaten übereinstimmende Bescheide heraus. Dann gibt es aber 
auch solche Fälle, da wird nur der Akt hin und her geschoben und es geht nichts weiter. Und das alles auf der Basis derselben EG-Richtlinie 2006/48. Das heißt, die EG hat die Phantasie, eine tolle Binnenmarktkonzeption zu entwickeln, setzt aber voraus, dass es funktionierende intensive Amtshilfeprozesse gibt. Die werden aber zurzeit gerade erst entwickelt. Für Österreich ergeben sich noch ganz spezielle Probleme daraus, dass diese Behörden bei den komplexen Finanzkonglomeraten und bei gemischten Unternehmungen untereinander vereinbaren, wer von ihnen die lead authority, also die hauptzuständige federfuihrende Behörde ist. Da drängt sich doch das Fragezeichen auf: Wie halten wir es mit dem gesetzlichen Richter? Die Zuständigkeiten werden vereinbart und das Ergebnis wird nicht einmal irgendwo kundgemacht.

Frowein: Ich möchte drei kurze Bemerkungen machen. Die erste schließt an die Thesen 5 und 10 von Herrn Classen an, zu denen ich sehr deutliche Zustimmung äußern möchte und nochmal unsere Diskussion zum Listingverfahren der UN gestern in Erinnerung rufen würde. Hier ist eigentlich präziser gesagt als vielleicht gestern teilweise herauskam, dass die Frage zu stellen ist, inwieweit dieser Rechtsanwendungsbefehl der da sein muss, seinerseits bedingt ist durch rechtsstaatliche Rechtsschutzregelungen. Meines Erachtens hat das das Gericht erster Instanz verkannt und meine Hoffnung ist, dass der EuGH es korrigieren wird. Die zweite Bemerkung betrifft den Bereich der UN-Direktverwaltung von Territorium. Das ist in beiden Referaten kurz angesprochen, aber nicht weiter erörtert worden, was keine Kritik ist. Hier haben wir es jetzt mit einem Vorgang zu tun, der in meinen Augen außerordentlich bedauerlich ist nämlich, dass der Europäische Gerichtshof für Menschenrechte in einer Zulässigkeitsentscheidung, die mit Mehrheit ergangen ist in dem Fall Berami Saramati, den Schutz der EMRK für den Bereich der im Kosovo tätigen KFOR-Truppen und man wird sagen müssen überhaupt für die Regelungen im Kosovo, ausgeschlossen hat, meines Erachtens ohne jede Not. Der Vorrang der UN-Resolutionen hätte festgestellt werden können, aber für die Ausfüllung der Lücken hätte die Europäische Menschenrechtskonvention bereitgehalten werden müssen. In demselben Zusammenhang möchte ich hinweisen auf einen wie ich finde beunruhigenden Vorgang, den ich vor einigen Jahren schon mal hinsichtlich der UNMIK-Verwaltung geprüft habe, nämlich die Rechtsetzung durch die UNMIK, die zum Teil nach Kriterien erfolgt, die überholt sind im europäischen Kontext. Beispiele sind Regulations der UNMIK, die den Rechtsschutz absolut verkürzen und auf diese Weise konventionsrechtlich nicht haltbar sind. Meine dritte Bemerkung ist nur ein kurzer abweichender Standpunkt gegenüber dem, 
was vorhin gesagt wurde hinsichtlich der Entwicklung der englischen und anderer Rechtsordnungen. Die Beeinflussung durch die EMRK und durch die Rechtsprechung von Kommission und Gerichtshof ist mit Händen zu-greifen und wird in Großbritannien allgemein anerkannt und von Gerichten laufend zitiert.

Wolfrum: Ich würde gerne als erstes dem Vorstand einen Dank aussprechen. Er hat uns gestern Nachmittag und heute Nachmittag, ich meine mit uns die mehr international Orientierten, geradezu verwöhnt. Aber mein Dank geht auch an die beiden Referenten für ihre vorzüglichen Referate.

Drei Punkte: Herrn Classen; ich habe einige Probleme mit Ihrer These 6. Sie haben sie mündlich etwas anders formuliert und dabei auch auf Art. 59 und 24 GG als Legitimationskette hingewiesen, wofür ich dankbar bin. Dieser Verweis ist auch völlig zutreffend. Ich frage mich aber, ob diese Legitimationskette ausreichend ist? Mehrere Gründe sprechen dagegen. Häufig sind diese Legitimationsakte alt und die Kompetenzen haben sich fortentwickelt im Sinne einer stärkeren Intensivierung. Müsste es nicht die Möglichkeit der Nachbesserung geben?

Zweiter Gesichtspunkt, der in der amerikanischen Literatur stärker problematisiert wird als in Deutschland: Es ist auch das Gesamtregime zu legitimieren und daran wirken nun sehr viele Staaten mit, deren Mehrheit nicht selbst demokratisch verfasst ist. Hat dies ein Demokratiedefizit zur Folge? Wenn ja, wie fangen wir es auf? Müssen wir hier nicht umdenken? Ich glaube, das was Sie in These 6 sagen, reicht dafür noch nicht aus. Ich würde Ihnen zugeben, wenn Sie fragen, haben Sie haben etwas besseres, ich habe es nicht.

Herr Biaggini zu Ihnen: Mir haben ausgesprochen gut gefallen, und insofern widerspreche ich Herrn von Bogdandy, Ihre Bezüge zum Bundesstaat. Ich glaube, dies wurde auch von anderen hervorgehoben, dieser Ansatz ist ein weiterführender und vor allem erfolversprechender Weg. Ich stimme zwar Ihrer These 17-19 nicht zu, dass das Auskunftssystem wirklich der Königsweg ist. Vielmehr würde ich vorschlagen, ein Streitbeilegungssystem zu entwickeln, das auf zwei Ebenen zu verorten wäre. Dieses Streitbeilegungssystem müsste zwei Dinge sicherstellen, die Effektivität der Rechtsanwendung und - und das ist der entscheidende Punkt - die Gleichförmigkeit der Rechtssprechung wie in einem Staat. Das bedingt eine Verortung auf nationaler, wie auch auf der jeweiligen zweiten oberen Ebene - ein typisches Element eines Mehrebenensystems.

Meiner letzter Punkt wieder zu Ihnen Herr Classen. Ich unterschreibe sehr nachdrücklich, was Sie in These 8-10 gesagt haben. Ich will nichts 
weiter dazu ausführen. Ich würde aber vorschlagen, dass Sie auch noch weiter auf die Durchsetzung gegenüber Einzelnen durch den ICC oder den Strafgerichtshof zu Jugoslawien hinweisen. Natürlich verstehen wir die Gerichte nicht als Durchsetzungselemente, aber im Völkerrecht sind sie es in diesen Fällen doch. Diese Gerichte setzen Kriegsvölkerrecht oder das entsprechende Recht in diesen Konflikten durch.

Ohler: Herr Classen, ich habe eine Frage an Sie und zwar speziell zu Ihrer These 16. Sie schreiben dort, die Übertragung von Hoheitsrechten auf ausländische Staaten wäre verfassungsrechtlich zulässig. Sind Sie auch bereit, die Konsequenz zu tragen, die wir gewöhnlich aus Art. 24 Abs. 1 GG ableiten, nämlich Anerkennung eines Vorranges des fremden Rechtes und Vorrang im Zweifel auch gegenüber innerstaatlichem Verfassungsrecht? $\mathrm{Zu}$ diesem Komplex noch eine Anmerkung. Ich hatte den Eindruck, dass in Ihren mündlichen Ausführungen gegenüber der These 16 erheblich zurückrudern, weil Sie nämlich grenzüberschreitende Observation und Nacheile als Jedermannsrechte qualifizieren. Mir erscheint diese Begründung zweifelhaft. Denn die Befugnisse, die die polizeilichen Kooperationsverträge verleihen, sollen Befugnisse im verwaltungsrechtlichen Sinne sein und nicht privatrechtliche Rechte. Wir sprechen im verfassungsrechtlichen Zusammenhang auch von Hoheitsrechtsübertragungen und nicht von Privatrechtsübertragungen. Betrachtet man die Polizeiorganisationsgesetze der Länder, erkennt man, dass Beamte ausländischer Staaten die Befugnisse genießen, die inländischen Beamten in Bayern, Thüringen, Sachsen zukommen. Also liegen echte Hoheitsausübungen vor, sofern es der jeweilige völkerrechtliche Vertrag ausdrücklich vorsieht. Noch eine Anmerkung zum Bankenaufsichtsrecht. Wir sind in diesem Rechtsbereich bereits viel weiter als im Polizeirecht. Im Vortrag wurde der Problemkreis der Vor-Ort-Kontrollen erwähnt. Das ist im Ergebnis vergleichsweise harmlos, weil die Kontrollen im Wesentlichen von beauftragten Wirtschaftsprüfungsgesellschaften ausgeübt werden. Auf Grund der letzten KWG-Novelle vom November 2006 besteht aber mittlerweile nach $\S 8 \mathrm{c}$ Abs. 3 KWG die Möglichkeit, dass die gesamte Einzelaufsicht über inländische Institute auf ausländische Behörden übertragen wird. Das ist beispielsweise für ein Institut wie die HypoVereinsbank relevant, die ja zum italienischen UniCredit-Konzern gehört. Hier wäre es nunmehr rechtlich möglich, dass die Banca d'Italia die vollständige Aufsicht ausübt. Die Aufsicht erfolgte dann natürlich nach italienischem Recht und nicht mehr nach deutschem.

Stelkens: Ich will nur eine Anmerkung machen, die sich letztlich auf die These 3 von Herrn Classen und die These 27 von Herrn Biaggini be- 
zieht und vielleicht auch auf die Bedenken von Herrn Sydow. Es geht um die Frage, wie allgemeine Rechtsgrundsätze des internationalen Verwaltungsrechts gefunden werden können: Jedenfalls begrenzt auf Europa haben wir für die Ermittlung allgemeiner Rechtsgrundsätze des Verwaltungsrechts eine recht taugliche Rechtserkenntnisquelle, die allerdings selten genutzt wird. Ich meine die Empfehlungen des Ministerkomitees des Europarates nach Art. 15b der Satzung des Europarates, die einerseits relativ ausführlich Verwaltungsrechtsstandards für die allgemeine Ausgestaltung von Verwaltungsverfahren normieren. Andererseits enthalten diese Empfehlungen aber z.B. auch Regelungsvorschläge zur Art und Weise von Privatisierungen, zur Ausgestaltung von Ausschreibungen oder zur korruptionsverhindernden Ausgestaltung des öffentlichen Dienstrechts. Die Empfehlungen fassen derartige Grundsätze in rechtsnormähnliche Formulierungen. Die Bindungswirkung dieser Empfehlungen scheint in den Mitgliedstaaten unterschiedlich beurteilt zu werden: Die skandinavischen Länder halten die Empfehlungen wohl sehr hoch und sehen darin etwas, an das sie ihr innerstaatliches Recht anpassen müssen; in Spanien scheint dies ähnlich zu sein. In Deutschland nimmt man die Empfehlungen dagegen meist nicht zur Kenntnis. Dennoch haben wir damit - seit den 1970er Jahren - Dokumente relativ umfangreicher Art, die letztlich Ausdruck eines geronnenen wertenden Rechtsvergleichs sind und die von den Mitgliedstaaten des Europarates auch mehr oder weniger offiziell akzeptiert worden sind, indem sie eben von ihren Ministern im Ministerkomitee verabschiedet wurden. In den Präambeln dieser Empfehlungen steht zudem immer ausdrücklich, dass die gefundenen Formulierungen einen „breiten Konsens" der beteiligten Staaten widerspiegeln. Daher scheint mir der Rückgriff auf diese Empfehlungen als eine vergleichsweise einfache Möglichkeit, allgemeine Rechtsgrundsätze des europäischen internationalen Verwaltungsrechts aufzufinden.

Biehler: Ich wollte auf den Rechtsschutz eingehen bei Herrn Classen in These 8. Einer gerichtlichen Kontrolle internationaler Standards sind Grenzen gesetzt. Diese Grenzen werden mit der Staatenpraxis in Verbindung gebracht. Hier ist der Bezug zwischen rechtlichen Maßstab und Gegenstand des rechtlichen Maßstabs zu betrachten. Ein kleiner Fall aus der internationalen Praxis: Nehmen wir an in dem Rahmen der Vereinten Nationen ist jemand zu Tode gekommen, nicht durch Außeneinwirkungen, sondern durch Nachlässigkeit im Rahmen des Dienstbetriebes. Die internationale Organisation, ist nicht bereit auf Grund der Immunitätsvorschriften in der Satzung der Vereinten Nation die Unterlagen dem Staat zur Verfügung zu stellen, der dies im Interesse der ge- 
schädigten Bürger, z.B. der Witwe, fordert. Da ist dann die Frage des nationalen oder überhaupt des Rechtsschutzes gestellt. Wenn man dann die Staatenpraxis als Maßstab der rechtlichen Grenzen sieht, im Sinne des Art. 38 I $1 \mathrm{~b}$ IGH-Satzung, dass hier die Staatenpraxis auch schon völkergewohnheitsrechtlich einen Maßstab bildet, entsteht das Problem, dass der rechtliche Maßstab durch das zu Beurteilende bestimmt wird.

Selbst im völkerrechtlichen schwebenden Bereich ist dies kein gangbarer Weg, sondern hier sollte die Unterscheidung zwischen Maßstab und Gegenstand des Maßstabs aufrechterhalten werden. Die gerichtliche Kontrolle die hier dann z.B. durch nationale Gerichte geleistet werden könnte, ist nötig, um den Barrieren, die die internationalen Organisationen gegenüber ihren Mitgliedsstaaten aufrechterhalten, im Wesentlichen Immunität, außenpolitische Entscheidungprärogrative usw., also der ganze Bereich der Privilegien die eben rechtliche Kontrolle ausschließen, an rechtlichen Maßstäben zu messen und keine rechtsfreien Räume unsererseits zu legitimieren oder gar zu konstruieren.

Khan: Ich hätte eine kurze Nachfrage zur These 16 von Herrn Classen. Lassen Sie mich begründen, warum ich Probleme habe mit Ihrer Aussage, dass die Übertragung von Hoheitsrechten auf ausländische Staaten - wenn überhaupt - dann nur in ganz begrenztem Ausmaße zulässig sei. Nicht weit von hier am Oberrhein gibt es seit Jahrhunderten eine Gemeinde, die sich beharrlich gegen die territorialen Begehrlichkeiten der Schweiz wehrt, nämlich das Dorf Büsingen; seit langer Zeit eine deutsche Exklave umgeben von Schweizer Hoheitsgebiet. Das wäre für uns heute vielleicht nicht mehr als eine pittoreske Fußnote der Geschichte, wenn es da nicht seit den sechziger Jahren des vergangenen Jahrhunderts einen umfassenden Vertrag gäbe, das Büsinger Vertragswerk, das der Schweiz weitgehende Verwaltungsbefugnisse für das Gemeindegebiet, als für deutsches Staatsgebiet, einräumt. Ich möchte vielleicht nicht so weit gehen, von einer Verwaltungszession zu sprechen; aber um mehr als lediglich punktuelle Hoheitsbefugnisse im Sinne Ihrer These geht es hier allemal. Etwas Ähnliches gilt übrigens auch für den ebenfalls von Ihnen angesprochenen deutsch-luxemburgischen Grenzraum. Die Verwaltungskooperation im hier bestehenden Kondominium hinsichtlich der Grenzflüsse geht weit über eine nur punktuelle Zusammenarbeit hinaus und umfasst auch die Wasserschutzpolizei und andere hoheitliche Befugnisse. Nun könnte man natürlich sagen, dies seien alles Kuriositäten, aber sie erschüttern natürlich irgendwie doch Ihre These. Wir alle wissen, dass das Grundgesetz Probleme mit dieser Fallkonstellation hat. Art. 24 sieht diese horizontale Internationalisierung der Ver- 
waltung eben nicht vor; erlaubt also eigentlich keine Übertragung deutscher Hoheitsgewalt auf andere Staaten. Aber können wir die Fakten wirklich ignorieren oder müssen wir uns nicht doch auf die Suche nach einer konstruktiven verfassungsrechtlichen Lösung für eine Situation machen, die in Zukunft wohl eher noch häufiger auftreten wird? Unbefriedigend jedenfalls wäre es, wenn wir die gesamten Verwaltungsbefugnisse der Schweiz hinsichtlich der Gemeinde Büsingen für verfassungswidrig ansehen würden - mit unabsehbaren Konsequenzen. Vielleicht könnten Sie dazu noch ein Wort zu sagen.

Häberle: Die beiden Referenten haben in ihren Schlussteilen eindrucksvoll an die „Aufgabe der Rechtswissenschaft" erinnert. Ich möchte positiv vermerken, dass es dem Vorstand gelungen ist, für alle acht Referate ein übergreifendes Motto zu finden: nämlich Leistungsfähigkeit - und ich füge hinzu - Leistungsanforderungen an die Wissenschaft vom Öffentlichen Recht. Daran sollten wir noch bei der einen oder anderen Selbstvergewisserung anknüpfen. Es ist mir hier nicht möglich zu fragen, wie es um die Leistungsfähigkeit der Verfassungsrechtswissenschaft in Osteuropa steht (Nachholbedarf seit 1989) oder auch im geliebten Italien. Ich möchte nur speziell auf die deutsche Wissenschaft vom Öffentlichen Recht aufmerksam machen, wie sie sich vor allem in der jungen Generation eindrucksvoll präsentiert. - Erstens: Dem Vorstand ist es wohl erstmals in der Tagungsgeschichte unserer Vereinigung geglückt - der Chronist Hans Peter Ipsen könnte mich korrigieren, „von oben“ natürlich -, das Völkerrecht in fast allen Gebieten zu einem zentralen Thema zu machen. Seine Präsenz und Virulenz war durchgängig. All dies einschließlich der Europäisierung und Internationalisierung und dies nicht nur bei den Fragen der „Globalisierung“, die wir jetzt als vernetzende Ausdehnung der „Welt" nicht nur zum Hinduismus, sondern auf alle Kontinente und Kulturen hin begreifen können. Da dies in vielen Detailfragen, zum Beispiel des internationalen Verwaltungsrechts geschah, war für mich die ganze Tagung ungemein lehrreich. - Zweitens: Wir haben zwar das nationale Verfassungsrecht, doch unser deutsches Grundgesetz "schrumpft", manche wollen es noch nicht wahrhaben. Das Grundgesetz ist nur noch eine „Schrumpfverfassung“, liebenswürdiger gesagt eine „Teilverfassung“. Das Bundesverfassungsgericht ist nur noch eine Teilverfassungsgerichtsbarkeit im Rahmen der EU und des Kontextes der EMRK. Aber welche „List der Vernunft“! All die Ideen und Prinzipien, die wir Deutschen fast perfektionistisch herausgearbeitet haben und die die Referenten auch vortrugen, nämlich die Aufwertung der Verfahren, die Arbeit am Rechtsschutz sowie der Demokratie und die hoch differenzierte Grundrechtswissenschaft: All dies 
können wir jetzt im Gespräch mit anderen nationalen Wissenschaftlergemeinschaften verfeinern und an die aktuellen Versuche des Völkerrechts und seine Wissenschaft weitergeben. - Drittens: Auch dieser Punkt ist ein Stück Entwicklungsgeschichte im Blick auf unsere Vereinigung. Wir sollten einige Klassiker des Verwaltungsrechts benennen und ihre Leistungen beim Thema Verwaltungsrecht als Steuerungswissenschaft würdigen. Ein Kollege hat schon Otto Mayer genannt. Es gibt aber auch Fleiner, ich meine den nach einer frühen Sprachregelung ,richtigen Fleiner", nämlich Fritz Fleiner, und es gibt jetzt noch den ,jüngeren Fleiner". Ich nenne überdies die großen Referate in Regensburg am zweiten Tag (1971). Sie haben vieles im Kern vorweggenommen. Ich meine die Referate der Kollegen Bachof und Brohm, in der Diskussion auch H.H. Rupp. Nicht nur im Nachgang sind die Referate der Kollegen Scholz und Schmidt-Aßmann zu erwähnen, auch das frühe „europäische Verwaltungsrecht" von J. Schwarze. - Im Ganzen: Einerseits darf sich unsere Wissenschaft als Akteur vor immensen Aufgaben ein gewisses Selbstbewusstsein leisten und sie verfügt auch über gutes Rüstzeug für deren Bewältigung, wie die ganze Tagung zeigt. Doch ist auch große Bescheidenheit angesagt. Die politische Macht hat ihre eigenen Wege und Irrwege, und die „List der Vernunft" erkennen wir nicht.

Biaggini: Sehr geehrte Kolleginnen, sehr geehrte Kollegen, ich bin Ihnen sehr dankbar für all die Anregungen aus der Diskussion und den vielfachen Zuspruch. Es ist eine schwierige Aufgabe, den Fragen und Bemerkungen in wenigen Minuten gerecht zu werden. Ich möchte versuchen, thematisch geordnet auf die Voten einzugehen, und hoffe, dass dabei nicht $\mathrm{zu}$ viel verloren geht.

Zuerst zum Begriff des „Internationalen Verwaltungsrechts“. Wenn der Eindruck entstanden sein sollte, dass das Kollisionsrecht aus dem Internationalen Verwaltungsrecht „herausgefallen“ ist: Dies war nicht meine Absicht. Das Kollisionsrecht ist und bleibt eine Komponente des „Internationalen Verwaltungsrechts“, genauso wie andere klassische Aspekte, etwa das „Recht der internationalen Verwaltung“ im Bereich internationaler Organisationen oder Regimes. Es geht um eine Erweiterung durch die Komponente „Annäherung Völkerrecht-Verwaltungsrecht". Diese habe ich im Vortrag in den Vordergrund gestellt.

Im Zusammenhang mit der Begrifflichkeit hat Herr Tietje die Frage gestellt: Was ist mit dem Territorialitätsprinzip? Ich habe diesen Begriff ausgeklammert und wollte auch bewusst nicht von einer „Entterritorialisierung" sprechen, weil es eine solche, so wie ich die Dinge sehe, in unserem Kontext nicht gibt. Vielmehr ist das relevante Territorium anders umschrieben. Dieses kann etwa das Gebiet zweier Staaten umfassen, die 
sich bilateral finden. Auch eine internationale Organisation ist letztlich, über die Mitgliedstaaten, territorial bestimmt. Was ich als Annäherung im Verhältnis „Verwaltungsrecht-Völkerrecht“ charakterisiert habe, findet auf einem bestimmten Territorium statt, das aber mehr als einen Staat umfasst.

Herr von Bogdandy, Frau Peters und Herr Kotzur haben das Thema „Konstitutionalisierung“ angesprochen: Ist dieser Vorgang vergleichbar mit dem, was sich im hier interessierenden Bereich des Verwaltungsrechts abspielt? Die Frage stellt sich nicht zuletzt vor dem Hintergrund der Diskussion in den Vereinigten Staaten von Amerika und in Italien. Ich sehe da durchaus Parallelen und denke, dass es bei der Übertragung von Rechtsfiguren auf die internationale Ebene um mehr geht als um eine blosse technische Angelegenheit. Wenn man die „Konstitutionalisierung" des Völkerrechts als Anreicherung der Völkerrechtsordnung um die „Idee der Verfassung“ und das, was dahinter steht (Grundrechte, Gewaltenteilung), charakterisieren kann, so könnte man die im Referat angesprochene "Ver-Verwaltungsrechtlichung" des Völkerrechts verstehen als eine Durchdringung des Völkerrechts mit der „Idee des Verwaltungsrechts“, d.h. mit den rechtstaatlichen Sicherungen, demokratischen Abstützungen und Rationalitätsgewährleistungen, die dem modernen Verwaltungsrecht eigen sind. Es gibt Parallelen, aber die Phänomene bewegen sich auf unterschiedlichen Ebenen. Insoweit besteht ein Unterschied zwischen dem verfassungsrechtlichen und dem verwaltungsrechtlichen Ansatz.

Ein weiterer Punkt - Frau Peters hat ihn angesprochen - betrifft den Einbezug der Bürgerinnen und Bürger, aber auch von privaten Verbänden. Ich denke, dass es nützlich ist, nach den beiden Grundphasen des Rechtsverwirklichungsprozesses zu unterscheiden, wie dies heute Vormittag auch im Referat von Herrn Appel geschehen ist. In der Phase der Regelbildung kann es Sinn machen, Private, ähnlich wie dies auf nationaler Ebene häufig geschieht, in den Rechtserzeugungsprozess einzubeziehen. Dies wird auf internationaler Ebene zum Teil schon praktiziert und kann durchaus noch ausgedehnt werden. In der zweiten Phase der Rechtsverwirklichung, der Phase der Umsetzung und Anwendung, spielt die Anhörung mehr auf der individuellen als auf der kollektiven Ebene. Auch hier kann man durchaus Parallelen zur nationalen Rechtsebene sehen, auch wenn bei der Übertragung von Rechtsfiguren Vorsicht am Platz ist und vorschnelle Analogien vermieden werden sollten. Ich denke, dass ich dies im Referat deutlich genug zum Ausdruck gebracht habe.

Was mich besonders gefreut hat, ist der Zuspruch, etwa von Herrn Schönberger, in Bezug auf den Vergleich zwischen der bundesstaat- 
lichen und der völkerrechtlichen Entwicklung. In der Tat: Wenn man zurückblendet in der Geschichte kann man nicht nur in der Schweiz, sondern auch in den Vereinigten Staaten von Amerika oder in Deutschland interessante Parallelphänomene entdecken. Ich möchte - als Schweizer - ein Beispiel aus der Schweizer Verfassungsgeschichte nennen. Als sich die in einem Staatenbund vereinten Kantone im Jahre $1848 \mathrm{zu}$ einem Bundesstaat zusammenschließen wollten, kam es in den Kantonen - mit einzelnen Ausnahmen - zu Volksabstimmungen. Die Abstimmungen fielen nicht durchweg positiv aus. Insgesamt acht Kantone sprachen sich gegen die Bundesverfassung und die damit verbundene Bundesstaatsgründung aus. Da die Eidgenossenschaft damals noch auf vertraglicher Grundlage beruhte (Bundesvertrag von 1815), hätte sich das Einstimmigkeitsprinzip durchsetzen müssen. Dies geschah nicht. Der Bundesstaat wurde gleichwohl gegründet. Sechs der acht Kantone, welche die Bundesverfassung von 1848 abgelehnt hatten, sagten auch bei der ersten Totalrevision im Jahre 1874 und bei der vor kurzem (1999) abgeschlossenen jüngsten Totalrevision nein. Es gibt somit sechs Kantone, die nie einer Bundesverfassung gesamthaft zugestimmt haben. Auch diese sechs Kantone sind heute - ganz selbstverständlich - im Bundesstaat dabei. Es wäre natürlich reizvoll, sich auszumalen, wie es sich verhielte, wenn diese Geschichte sich in heutigen Tagen eine Ebene „höher" zutragen würde: im Zusammenhang mit dem EU-Verfassungsvertrag oder jetzt mit dem Reformvertrag. Was wäre wenn? Ich will damit nicht sagen, dass man unbesehen Parallelen zu 1848 ziehen sollte. Aber es gibt im Verhältnis Bundesstaat-EU viel mehr an Vergleichsmöglichkeiten, als man beim ersten Blick denken mag. Gerade die Schweiz ist als Vergleichsbeispiel prädestiniert, zumal der schweizerische Bundesstaat noch heute gewisse staatenbündische Züge zeigt, wie man mit verschiedenen Beispielen belegen könnte. Für die Beobachtung und Analyse der Annäherung von Völkerrecht und Verwaltungsrecht scheint mir der Vergleich mit bundesstaatlichen Entwicklungen in der Tat ein interessanter Ansatz zu sein.

Was die Übertragbarkeit von Rechtsfiguren angeht, die in der Diskussion verschiedentlich auf Skepsis stiess, bin ich etwas optimistischer. Diese Zuversicht gründet nicht zuletzt darin, dass es hier um Prozesse geht, die langfristig ablaufen und immer auch räumlich lokalisiert werden müssen. Man darf nicht erwarten, dass die „Ver-Verwaltungsrechtlichung" des Völkerrechts sich alsgleich in hohem Tempo auf globaler Ebene ausbreitet. Resultate stellen sich leichter im kleinen Rahmen ein: auf europäischem Boden, vielleicht auch zunächst bloss im bilateralen Verhältnis. Hier lassen sich manche Dinge sehr viel rascher und ohne große Komplikationen verwirklichen. Dabei spielt, wie ich im Referat 
betont habe, die Grundkategorie des Vertrauens eine ganz zentrale Rolle. Angesichts der grossen Bedeutung der Kategorie des Vertrauens ist es eine sehr wichtige Aufgabe von Praxis und Wissenschaft, die Voraussetzungen dafür zu schaffen, dass Vertrauen sich bilden und eine breite Basis finden kann. Auf diese Weise können Annäherungsprozesse sich allmählich auch über zunächst begrenzte Räume hinaus weiterentwickeln. Man darf mithin die Faktoren „Zeit“ und „Raum“ nicht aus den Augen verlieren.

Ganz zuletzt komme ich auf die erste Frage zu sprechen, die für mich die schwierigste ist. Herr Ress hat sie gestellt: Warum nicht den Mut aufbringen zu sagen, dass es sich beim „Internationalen Verwaltungsrecht" um eine ganz neue Kategorie handelt? Die soeben erwähnten Faktoren "Zeit" und „Raum" sind da für mich sicherlich mit ausschlaggebend, und dann gibt es auch einen biographischen Grund. Ich komme nicht vom Völkerrecht her und habe die hier diskutierten Fragen in erster Linie durch die Brille des Staats- und Verwaltungsrechts und weniger durch die Brille des Völkerrechts betrachtet. Ich habe im Referat bewusst davon abgesehen, mich für eine Verselbstständigung des „Internationalen Verwaltungsrechts“, im Sinne eines solchen "großen Wurfs“, auszusprechen. Und ich frage mich, ob es wirklich in diese Richtung weitergehen kann und soll. Am Schluss des Referats habe ich für eine Integration des "Internationalen Verwaltungsrechts“ in das Allgemeine Verwaltungsrecht plädiert. Ich möchte hier nochmals betonen, dass mir dabei nicht ein Allgemeines Verwaltungsrecht mit einem rein nationalen Zuschnitt vorschwebt, sondern ein Allgemeines Verwaltungsrecht, das offen ist für viele Rechtsquellen, das auch europarechtliche Bezüge integriert (in der Schweiz natürlich etwas weniger), das weiter auch internationale, völkerrechtlich fundierte Elemente einbezieht und somit, vom Ansatz her, nicht rechtsquellengebunden, sondern rechtsquellenneutral und insofern umfassend ist.

Classen: Ich darf mich zunächst einmal ganz herzlich bedanken für die zahlreichen Anregungen, auch für die insgesamt recht freundliche Aufnahme meiner Überlegungen. Der Begriff des „Internationalen Verwaltungsrechts" war mir mit der Themenstellung vorgegeben. Ich habe diese auch sehr gerne bearbeitet, aber dafür, wie die Begrifflichkeiten wirklich zu fassen sind, bin ich nicht verantwortlich. In der Sache hoffe ich deutlich gemacht zu haben, dass es insgesamt um ein sehr vielfältiges Thema geht. Darüber, ob man bestimmte Aspekte vielleicht anders einordnen kann, kann man diskutieren. Insgesamt, so glaube ich, ist es schwierig, ein eingeschlossenes System entwickeln; es gibt eben einfach viele verschiedene Fragestellungen. 
Teilweise kann man die Entwicklungen, die ich beschrieben habe, durchaus als Beleg für eine Konstitutionalisierung des Völkerrechts ansehen, aber es gibt natürlich erhebliche Unterschiede. Es ist wohl selbstverständlich, dass man leichter für einen grenzüberschreitenden Wasserverband ein rechtsstaatliches Verwaltungsregime entwickeln kann, etwa nach dem Vorbild der Rheinschifffahrtskommission, als rechtsstaatliche Grundsätze für die Arbeit des Sicherheitsrats formulieren. Aber auch insofern ist zu differenzieren. Wenn es um Entscheidungen unmittelbar des Sicherheitsrats selber geht, darüber ist ja gestern diskutiert worden, kann ich eine gewisse Zurückhaltung durchaus verstehen. Wenn dagegen lokale Entscheidungen im Kosovo oder anderswo getroffen werden, stellt eine gerichtliche Überprüfung die Autorität des Sicherheitsrats eigentlich nicht in Frage. Ebenso missachtet eine Kontrolle an menschenrechtlichen Maßstäben den Vorrang der UN-Charta nach Art. 103. Von daher bin auch ich von der Entscheidung des EGMR in der Rechtssache Bahrami und Seramati sehr enttäuscht. Aber gut, man muss sie natürlich zur Kenntnis nehmen.

Es ist mehrfach nach der demokratischen Legitimation gefragt worden, nach der Rolle von privaten Akteuren. Die Welt ist natürlich zunächst einmal so wie sie ist. Man kann sich sicherlich wünschen, dass sie eine andere wäre. Fragt man nach Alternativen, konkret etwa, ob das, was auf europäischer Ebene praktiziert wird, nämlich die Entwicklung parlamentarischer Strukturen, auch auf internationaler Ebene sinnvoll ist, so möchte ich aus den verschiedensten Gründen verneinen. Man merkt das schon daran, dass dort, wo es parlamentarische Versammlungen gibt, wie etwa bei der NATO, diese nur eine begrenzte Wirkung entfalten. Eine wesentliche parlamentarische Funktion besteht ja darin, verschiedene Interessen zu integrieren; dies kann bei Fachorganisationen nicht so richtig funktionieren. Letztlich ist aus meiner Sicht wichtig, und insoweit wurde die entsprechende These in der Diskussion nicht ganz vollständig angesprochen, dass gegebenenfalls auch auf nationaler Ebene Korrekturmöglichkeiten bestehen. Ich weiß, dass der Bundestag das ist gestern ja schon deutlich geworden - bei der Bewältigung der Herausforderungen durch die europäische Integration nicht sehr überzeugend arbeitet. Das liegt aber nicht an Europa, sondern am Bundestag, wie die Beispiele des britischen und des dänischen Parlaments belegen. Man kann sich durchaus vorstellen, dass man hier entsprechend „aufrüstet", dass hier etwas mehr Transparenz hineinkommt, und dann dürfte das Ganze vielleicht auch etwas besser funktionieren.

Einen kleinen Vorbehalt möchte ich mit Blick auf die diskutierte Konstitutionalisierung des Völkerrechts anmelden, auch wenn das etwas gegen einen Teil des Vorstands geht. Ich habe Schwierigkeiten bei der 
Begrifflichkeit „Vollzug von Völkerrecht", weil die völkerrechtlichen Verpflichtungen - das ist auch angesprochen worden - gerade nicht so ausgestaltet sind, dass ein Verwaltungsbeamter sie gleich umsetzen kann. Völkerrecht ist vielfach nicht unmittelbar anwendbar, und zwar nicht nur aus formalen, sondern auch aus inhaltlichen Gründen, weil vielfach Spielräume für die nationale Ebene bestehen. Deswegen treibt mich auch die angesprochene Sorge nicht um, dass es bei der Internationalisierung ähnliche Abwehrreaktionen wie bei der Europäisierung des Verwaltungsrechts geben könnte. Die Durchgriffswirkung des Europarechts ist eine fundamental andere als die Wirkung des Völkerrechts, und auch der inhaltliche Einfluss auf nationale Regelungskonzepte ist sicherlich mit einigen Ausnahmen - insgesamt deutlich geringer.

Damit bin ich schon bei dem verschiedentlich angesprochenen Thema Europa, das aus meiner Sicht zwei Facetten hat. Zunächst stellt sich die Frage, ob nicht vieles von dem, was bei uns zu vollziehen ist, über die europäische Union vermittelt wird, ob also internationale Entwicklungen vermittelt über die europäische Union bei uns ankommen, und ob man deswegen nicht auch für die Europäische Union entsprechende Regelungen, Mechanismen usw. entwickeln müsste. Das ist vollkommen richtig. Ich habe das bewusst ausgeklammert, weil ich in $45 \mathrm{Mi}-$ nuten nicht alle Probleme ansprechen konnte. Erst recht gilt das, soweit verschiedene Beispiele unmittelbar aus dem europäischen Recht erwähnt wurden, also Inspektionen, Bankenaufsicht oder ähnliches. Das geht auf das Gemeinschaftsrecht zurück, und ich denke, dass sich dieses aus mehreren Gründen stark von den internationalen Entwicklungen unterscheidet: es gibt eine gemeinsame Gerichtsbarkeit und ein relativ etabliertes Regelset von allgemeinen Rechtsätzen. Hier stellen sich strukturell völlig andere Probleme, weswegen ich darauf nicht eingehen möchte.

Zur Frage, woher man die allgemeinen Rechtsgrundsätze schöpft, ob es überhaupt möglich ist, auf internationaler Ebene allgemeine internationale Standards zu entwickeln: Zunächst einmal gibt es als Grundlage die Menschenrechte, und zwar nicht nur als abstrakte Regeln auf dem Papier. Es gibt den Menschenausschuss der Vereinten Nationen, der regelmäßig entsprechende Entscheidungen trifft, allgemeine Bemerkungen macht usw. Da verfügt man über einen großen Fundus. Es gibt die diversen internationalen Dienstgerichte, die natürlich nur für einen punktuellen Bereich, dort aber immerhin wichtige Arbeit leisten, genauso wie die angesprochenen Strafgerichtshöfe zur Entwicklung des Verfahrensrechts beitragen. Natürlich macht es Probleme, aus weltweiten Traditionen heraus gewisse Standards zu destillieren, aber die genannten Institutionen machen dies mit - soweit ich es beobachten 
kann - einem so großen Erfolg, dass man hier durchaus optimistisch sein kann. Und wenn es Unterschiede in den Rechtstraditionen gibt, dann betreffen diese, und das ist eine allgemeine Erfahrung der Rechtsvergleichung, wesentlich stärker die Frage der methodischen Herleitung und ähnliches als das praktische Ergebnis. Wenn man also einfach konkrete Ergebnisse erwartet und nicht die Frage nach der Konsistenz einer Theorie stellt, die ein internationales Gericht oder eine ähnliche Institution seinen Entscheidungen zu Grunde legt, dann kann man durchaus optimistisch sein.

Zur Frage des Territorialitätsprinzipes. Es ist sicherlich richtig, dass man insoweit zu Zeiten von Otto Meyer ganz andere Vorstellungen hatte als heute. Wenn man sein Lehrbuch des allgemeinen Verwaltungsrecht liest, das in der ersten Auflage auch noch bestimmte einschlägige Passagen aufwies, die in der heute meist zitierten dritten Auflage nicht mehr enthalten sind, wird aber deutlich, das das Prinzip auch damals eine Regel mit Ausnahmen darstellte. Die größere Bedeutung der Ausnahmen, die wir heute wahrnehmen, hängt wohl auch damit zusammenhängen, dass nunmehr die entsprechenden Problemstellungen sehr viel zahlreicher sind. Ich habe dies ja kurz angesprochen. Früher war vieles nicht geregelt, und allein deswegen waren die Grenzen offen. Heute sind alle Lebensbereiche geregelt, viel dichter als früher, und dann entstehen viel mehr Probleme, die man zu bewältigen hat. Dass man bei den klassischen völkerrechtlichen Regeln zur Unterscheidung zwischen der jurisdiction to enforce einerseits und der jurisdiction to prescribe andererseits die Akzente grundsätzlich anders setzen muss als früher, sehe ich nicht.

Schließlich noch eine Bemerkung zu These 16, die mehrfach angesprochen wurde. Zunächst einmal der Hinweis auf die Vergleichbarkeit mit den privaten Rechten bei Observation und Nacheile. Hier ging es mir nicht darum, und die schriftliche Form macht das hoffentlich auch deutlicher, als es jetzt vielleicht im mündlichen Vortrag angekommen ist, zu behaupten, dass es sich hier um private Rechtstitel handelt. Ich habe einfach eine Parallelwertung angestellt. Wenn man ausländischen Hoheitsträgern bestimmte, hoheitlich bleibende Rechte zugesteht, die in ähnlicher Form zumindest rein praktisch gesprochen auch Private bei uns haben, ist das anders zu bewerten als wenn es um Rechte geht, die auch bei uns nur einem Hoheitsträger zustehen. Büsingen speziell stellt aus meiner Sicht auch einen eng begrenzten Ausnahmefall dar. Dass die These 16 als zu eng kritisiert wurde, erstaunt mich im Lichte der wissenschaftlichen Diskussion um diese Norm. Die meisten sagen ja, dass eine Übertragung von Hoheitsrechten auf ausländische Staaten überhaupt nicht zulässig ist. Ich glaube, dass man Art. 24 Abs. 1 und 1a GG 
schon insoweit erst nehmen muss, dass wenn die Übertragung an zwischenstaatliche Einrichtungen und grenznachbarschaftliche Einrichtungen geregelt ist und sonst nichts, man nicht einfach sagen kann, dass alles andere auch geht. Dann wäre Art. 24 wertlos. Aber umgekehrt kann man eben auch nicht behaupten, dass die Norm, die internationale Kooperation erleichtern wollte, etwas verbietet, was es an internationaler Kooperation immer schon gegeben hat; das würde diese Norm natürlich genauso verfehlen. Daher denke ich, dass wir hier nicht weit auseinander sind.

Oppermann: Ich habe die schöne Aufgabe, dem Vorstand zu danken. Ihnen Herr Schoch, Ihnen Herr Pieroth, Herrn Ferdinand Kirchhof - er ist gerade nicht im Raum - in absentia, aber last but not least, Ihnen lieber Herr Würtenberger! Sie als ortszuständiges Mitglied haben ganz besonders zu unserem Wohlergehen beigetragen. Dem Vorstand insgesamt gilt vor allen Dingen Dank für zwei außerordentlich gut und interessant ausgewählte Themata für die Referate. Wir haben zwei Tage mit lebhaften, kontroversen, tiefen Diskussionen hinter uns. Aber vielleicht das Wichtigste: Es war niemals langweilig! Das ist für die Teilnehmer immer das Schönste an solchen Tagungen. Gleichzeitig Dank auch für das reichhaltige Rahmenprogramm! Nach allen Rückmeldungen ist es sehr gut angekommen. Und Dank natürlich den Helferinnen und Helfern, soweit sie hier sind, sonst bitte ich es weiterzugeben. Zuletzt besonderer Dank einem ganz besonderem „Helfer“, nämlich Freiburg im Breisgau und seiner Umgebung! Wer früher einmal in der Schule oder zum Studium in Freiburg gelebt hat, kommt jedes Mal nur allzu gerne zurück. Auch mit dem Wettergott, lieber Herr Schoch, waren Sie offensichtlich im Bunde. Eines habe ich allerdings vermisst. Niemand hat mir zu erklären vermocht, wieso die berühmten Freiburger Bächle entlang der Straßen kein Wasser mehr führen. Das kann vielleicht nachgeholt werden. Nochmals zusammengefasst: herzlichen Dank im Namen von uns allen! Das Programm der Tagung einschließlich einiger noch zu erwartender Höhepunkte ist noch nicht zu Ende, aber meine Dankesrede. 
